\title{
Stereoselective Syntheses of $\gamma, \delta$-Bifunctionalized Homoallylic Alcohols and Ethers via Chemoselective Allyl Addition to Aldehydes
}

\author{
Jichao Chen, Shang Gao and Ming Chen* \\ Department of Chemistry and Biochemistry
}

Auburn University

E-mail: mzc0102@auburn.edu

Supporting Information: Experimental Procedures, Tabulated Spectroscopic Data, ${ }^{1} \mathrm{H}$ and

${ }^{13} \mathrm{C}$ Spectra of New Compounds 
General Experimental Details. All reaction solvents were purified before use. Dichloromethane, 1,2-dichloroethane and toluene were purified by passing through a solvent column composed of activated A-1 alumina. Unless indicated otherwise, all reactions were conducted under an atmosphere of argon using flame-dried or oven-dried $\left(120{ }^{\circ} \mathrm{C}\right)$ glassware. The term "concentrated under reduced pressure" refers to the removal of solvents and other volatile materials using a rotary evaporator with the water bath temperature below $30{ }^{\circ} \mathrm{C}$, followed by the removal of residual solvents at high vacuum $(<0.2$ mbar $)$.

Proton nuclear magnetic resonance ( ${ }^{1} \mathrm{H}$ NMR) spectra were acquired on commercial instruments (400 and $600 \mathrm{MHz}$ ) at Auburn University NMR facility. Carbon-13 nuclear magnetic resonance $\left({ }^{13} \mathrm{C}\right.$ NMR) spectra were acquired at 101 and $151 \mathrm{MHz}$. The proton signal for the residual non-deuterated solvent $\left(\delta 7.26\right.$ for $\left.\mathrm{CHCl}_{3}\right)$ was used as an internal reference for ${ }^{1} \mathrm{H}$ NMR spectra. For ${ }^{13} \mathrm{C}$ NMR spectra, chemical shifts are reported relative to the $\delta 77.36$ resonance of $\mathrm{CHCl}_{3}$. Coupling constants are reported in $\mathrm{Hz}$. High-resolution mass spectra were recorded on a commercial high-resolution mass spectrometer via the Micro Mass/Analytical Facility operated by the College of Chemistry and Biochemistry, Auburn University.

Analytical thin layer chromatography (TLC) was performed on Kieselgel 60 F254 glass plates precoated with a $0.25 \mathrm{~mm}$ thickness of silica gel. The TLC plates were visualized with UV light and/or by staining with Hanessian solution (ceric sulfate and ammonium molybdate in aqueous sulfuric acid) or $\mathrm{KMnO}_{4}$. Column chromatography was generally performed using Kieselgel 60 (230-400 mesh) silica gel, typically using a 50-100:1 weight ratio of silica gel to crude product. 


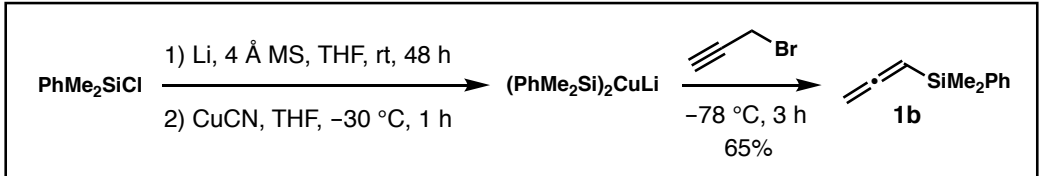

Dimethyl(phenyl)(propa-1,2-dien-1-yl)silane 1b: To a suspension of lithium wire (41.2 mmol, 14 equiv) and $4 \AA$ molecular sieves $(1 \mathrm{~g})$ in anhydrous THF $(12 \mathrm{~mL})$ at ambient temperature under an argon atmosphere was added dimethylphenylchlorosilane (5.88 mmol, 2 equiv). The mixture was stirred for $48 \mathrm{~h}$ at ambient temperature. The resulting reddish-brown solution was transferred into a slurry of $\mathrm{CuCN}$ (2.94 mmol, 1 equiv) in anhydrous THF $(12 \mathrm{~mL})$ at $-30{ }^{\circ} \mathrm{C}$ under an argon atmosphere. The mixture was stirred for $1 \mathrm{~h}$ and then cooled to $-78^{\circ} \mathrm{C}$. Propargyl bromide (2.94 mmol, 1 equiv) was added to the resulting silyl cuprate and the resulting mixture was stirred at $-78{ }^{\circ} \mathrm{C}$ for $3 \mathrm{~h}$. Then diethyl ether $(50 \mathrm{~mL})$ was added and the mixture was filtered through a pad of silica gel. The solvent was removed under reduced pressure. Purification of the crude reaction product was performed by flash column chromatography with hexane to give product $\mathbf{1 b}$ as colorless oil in $65 \%$ yield $(330 \mathrm{mg}) .{ }^{1} \mathrm{H} \mathrm{NMR}\left(600 \mathrm{MHz}, \mathrm{CDCl}_{3}\right) \delta 7.55-7.57(\mathrm{~m}$, 2H), $7.37-7.38$ (m, 3H), 5.05 (t, $J=7.1 \mathrm{~Hz}, 1 \mathrm{H}), 4.40$ (d, $J=7.1 \mathrm{~Hz}, 2 \mathrm{H}), 0.38(\mathrm{~s}, 6 \mathrm{H})$. ${ }^{13} \mathrm{C}$ NMR $\left(151 \mathrm{MHz}, \mathrm{CDCl}_{3}\right) \delta 213.7,138.6,134.0,129.5,128.1,79.7,67.8,-2.1$. HRMS $\left(\mathrm{EI}^{+}\right): \mathrm{m} / \mathrm{z}$ for $\mathrm{C}_{11} \mathrm{H}_{14} \mathrm{Si}[\mathrm{M}]^{+}$calcd. 174.0865, found 174.0862. The spectroscopic data for this compound were in agreement with previously reported data.

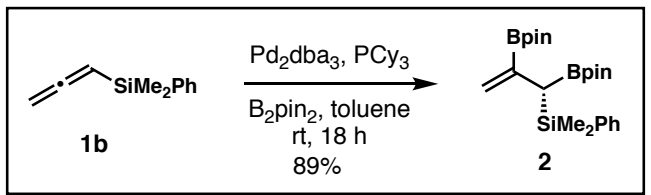

(1,2-Bis(4,4,5,5-tetramethyl-1,3,2-dioxaborolan-2-yl)allyl)dimethyl(phenyl)silane (2): In an Ar-filled glove box, $\mathrm{Pd}_{2}(\mathrm{dba})_{3}(0.0025 \mathrm{mmol}, 2.5 \mathrm{~mol} \%), \mathrm{PCy}_{3}(0.006 \mathrm{mmol}, 6$ mol \%), allene $1 \mathbf{b}$ ( $0.1 \mathrm{mmol}, 1.0$ equiv), $\mathrm{B}_{2} \operatorname{Pin}_{2}(0.12 \mathrm{mmol}, 1.2$ equiv), toluene $(0.5 \mathrm{~mL})$ and a Teflon-coated magnetic stirring bar were sequentially added into a 1-dram vial. After stirring at ambient temperature for $18 \mathrm{~h}$, the reaction mixture was filtered through a pad of silica gel. The solution was concentrated under reduced pressure. Purification of the crude reaction product was performed by flash column chromatography (gradient elution with hexane and ethyl acetate) to give product 2 as yellowish oil in $89 \%$ yield (38 mg). ${ }^{1} \mathrm{H}$ NMR (600 MHz, $\left.\mathrm{CDCl}_{3}\right) \delta 7.54-7.55$ (m, 2H), $7.29-7.30$ (m, 3H), 5.75 (s, $1 \mathrm{H}), 5.68(\mathrm{~s}, 1 \mathrm{H}), 2.04(\mathrm{~s}, 1 \mathrm{H}), 1.14(\mathrm{~s}, 12 \mathrm{H}), 1.12(\mathrm{~s}, 6 \mathrm{H}), 1.11(\mathrm{~s}, 6 \mathrm{H}), 0.34(\mathrm{~s}, 3 \mathrm{H}), 0.30$ (s, 3H). ${ }^{13} \mathrm{C}$ NMR $\left(151 \mathrm{MHz}, \mathrm{CDCl}_{3}\right) \delta$ 139.3, 134.6, 129.0, 128.2, 127.6, 83.6, 83.0, 25.3, 
25.2, 25.06, 25.05, 21.4, -2.5, -2.7. HRMS $\left(\mathrm{ESI}^{+}\right)$: $\mathrm{m} / \mathrm{z}$ for $\mathrm{C}_{23} \mathrm{H}_{39} \mathrm{~B}_{2} \mathrm{O}_{4} \mathrm{Si}[\mathrm{M}+\mathrm{H}]^{+}$calcd. 429.2804, found 429.2813 .

\section{Procedure of a $\mathbf{2} \mathbf{~ m m o l - s c a l e}$ reaction for the synthesis of allylboronate 2:}

In an Ar-filled glove box, $\mathrm{Pd}_{2}(\mathrm{dba})_{3}(46 \mathrm{mg}, 0.05 \mathrm{mmol}, 2.5 \mathrm{~mol} \%), \mathrm{PCy}_{3}(34 \mathrm{mg}, 0.12$ mmol, $6 \mathrm{~mol} \%$ ), allene $\mathbf{1 b}$ (350 mg, $2 \mathrm{mmol}, 1.0$ equiv), $\mathrm{B}_{2} \mathrm{Pin}_{2}$ (610 mg, 2.4 mmol, 1.2 equiv), toluene $(5 \mathrm{~mL})$ and a Teflon-coated magnetic stirring bar were sequentially added into a $20 \mathrm{~mL}$ vial. After stirring at ambient temperature for $24 \mathrm{~h}$, the reaction mixture was filtered through a pad of silica gel. The filtrate was concentrated under reduced pressure. Purification of the crude reaction product was performed by flash column chromatography (gradient elution with hexane and ethyl acetate) to give product $\mathbf{2}$ as yellowish oil in $91 \%$ yield $(780 \mathrm{mg})$.
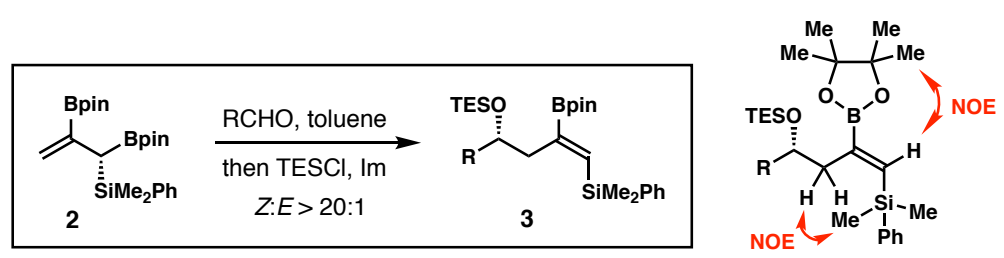

General procedure for the syntheses of compounds 3 using aldehyde allylation with reagent 2: In an Ar-filled glove box, to a reaction vial containing a Teflon-coated magnetic stirring bar was added freshly distilled aldehydes $(0.1 \mathrm{mmol}, 1.0$ equiv, if it is a liquid). A solution of allylboronate $2(0.12 \mathrm{mmol}, 1.2$ equiv) in toluene $(0.2 \mathrm{~mL})$ was added to the vial. The mixture was stirred for $12-48 \mathrm{~h}$ at ambient temperature. After complete consumption of the aldehyde, $\mathrm{TESCl}(0.2 \mathrm{mmol}, 2.0$ equiv) and imidazole $(0.2$ mmol, 2.0 equiv) were added at $0{ }^{\circ} \mathrm{C}$. The reaction mixture was then warmed to ambient temperature and stirred for additional $12 \mathrm{~h}$. $\mathrm{Et}_{2} \mathrm{O}(2 \mathrm{~mL})$ was added and the mixture was filtered through a pad of silica gel. The solution was concentrated under reduced pressure and the residue was purified by flash column chromatography (gradient elution with hexane and ethyl acetate) to give product $\mathbf{3}$. The olefin geometry of $\mathbf{3}$ was assigned as $Z$ by NOE studies.

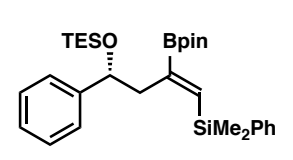

(R,Z)-((4-(Dimethyl(phenyl)silyl)-1-phenyl-3-(4,4,5,5-tetramethyl-1, 3,2-dioxaborolan-2-yl)but-3-en-1-yl)oxy)triethylsilane (3a) Prepared

according to the general procedure. The crude mixture was purified by column chromatography with hexane and ethyl acetate to give the title compound as colorless oil in 90\% yield (47 mg). ${ }^{1} \mathrm{H}$ NMR (600 MHz, $\left.\mathrm{CDCl}_{3}\right) \delta 7.47-7.49(\mathrm{~m}, 2 \mathrm{H})$, 
$7.31-7.33(\mathrm{~m}, 3 \mathrm{H}), 7.23-7.27(\mathrm{~m}, 4 \mathrm{H}), 7.18-7.20(\mathrm{~m}, 1 \mathrm{H}), 6.68(\mathrm{~s}, 1 \mathrm{H}), 4.92(\mathrm{dd}, J=$ 6.7, $6.6 \mathrm{~Hz}, 2 \mathrm{H}), 2.70(\mathrm{dd}, J=12.9,7.5 \mathrm{~Hz}, 1 \mathrm{H}), 2.49$ (dd, $J=12.9,5.8 \mathrm{~Hz}, 1 \mathrm{H}), 1.30$ (s, $6 \mathrm{H}), 1.29$ (s, 6H), 0.85 (t, $J=8.0 \mathrm{~Hz}, 9 \mathrm{H}), 0.42-0.53(\mathrm{~m}, 6 \mathrm{H}), 0.31(\mathrm{~s}, 3 \mathrm{H}), 0.30(\mathrm{~s}, 3 \mathrm{H})$. ${ }^{13} \mathrm{C}$ NMR $\left(151 \mathrm{MHz}, \mathrm{CDCl}_{3}\right) \delta 147.1,146.2,139.8,134.2,129.0,128.1,128.0,127.0$, 126.3, 83.8, 74.6, 47.4, 25.4, 25.2, 7.2, 5.1, -0.48, -0.55. HRMS $\left(\mathrm{ESI}^{+}\right): \mathrm{m} / \mathrm{z}$ for $\mathrm{C}_{30} \mathrm{H}_{47} \mathrm{BO}_{3} \mathrm{Si}_{2} \mathrm{Na}[\mathrm{M}+\mathrm{Na}]^{+}$calcd. 545.3055, found 545.3030.

\section{Procedure of a $1 \mathrm{mmol}-\mathrm{scale}$ reaction for the synthesis of 3a:}

In an Ar-filled glove box, to a reaction vial containing a Teflon-coated magnetic stirring bar was added freshly distilled benzaldehyde (106 mg, $1 \mathrm{mmol}, 1.0$ equiv,). A solution of allylboronate 2 (514 mg, $1.2 \mathrm{mmol}, 1.2$ equiv) in toluene ( $3 \mathrm{~mL}$ ) was added to the vial. The mixture was stirred for $24 \mathrm{~h}$ at ambient temperature. After complete consumption of the aldehyde, TESCl ( $2 \mathrm{mmol}, 2.0$ equiv), imidazole ( $2 \mathrm{mmol}, 2.0$ equiv) and $\mathrm{CHCl}_{3}$ (1 $\mathrm{mL}$ ) were added at $0{ }^{\circ} \mathrm{C}$. The reaction mixture was then warmed to ambient temperature and stirred for additional $12 \mathrm{~h}$. $\mathrm{Et}_{2} \mathrm{O}(5 \mathrm{~mL})$ was added and the mixture was filtered through a pad of silica gel. The solution was concentrated under reduced pressure and the residue was purified by flash column chromatography (gradient elution with hexane and ethyl acetate) to give product 3 as yellowish oil in $82 \%$ yield (428 $\mathrm{mg}$ ).

Methyl $(R, Z)-4-(4-($ dimethyl(phenyl)silyl)-3-(4,4,5,5-tetrameth
yl-1,3,2-dioxaborolan-2-yl)-1-((triethylsilyl)oxy)but-3-en-1-yl)
benzoate (3b) Prepared according to the general procedure. The crude mixture was purified by column chromatography with hexane and ethyl acetate to give the title compound as colorless oil in $81 \%$ yield $(47 \mathrm{mg}) .{ }^{1} \mathrm{H}$ NMR $(600 \mathrm{MHz}$, $\left.\mathrm{CDCl}_{3}\right) \delta 7.92(\mathrm{~d}, J=8.2 \mathrm{~Hz}, 2 \mathrm{H}), 7.43-7.44(\mathrm{~m}, 2 \mathrm{H}), 7.26-7.33(\mathrm{~m}, 5 \mathrm{H}), 6.69(\mathrm{~s}, 1 \mathrm{H})$, $4.96(\mathrm{dd}, J=6.7,6.6 \mathrm{~Hz}, 2 \mathrm{H}), 3.90(\mathrm{~s}, 3 \mathrm{H}), 2.66(\mathrm{dd}, J=13.0,7.4 \mathrm{~Hz}, 1 \mathrm{H}), 2.45$ (dd, $J=$ $13.0,5.9 \mathrm{~Hz}, 1 \mathrm{H}), 1.28(\mathrm{~s}, 12 \mathrm{H}), 0.83(\mathrm{t}, J=7.9 \mathrm{~Hz}, 9 \mathrm{H}), 0.40-0.52(\mathrm{~m}, 6 \mathrm{H}), 0.28(\mathrm{~s}$, $3 \mathrm{H}), 0.26(\mathrm{~s}, 3 \mathrm{H}) .{ }^{13} \mathrm{C} \mathrm{NMR}\left(151 \mathrm{MHz}, \mathrm{CDCl}_{3}\right) \delta 167.6,151.6,147.8,139.6,134.2,129.6$, $129.1,128.8,128.0,126.2,83.9,74.2,52.4,47.1,25.3,25.2,7.2,5.0,-0.5,-0.6$. HRMS $\left(\mathrm{ESI}^{+}\right): \mathrm{m} / \mathrm{z}$ for $\mathrm{C}_{32} \mathrm{H}_{53} \mathrm{BNO}_{5} \mathrm{Si}_{2}\left[\mathrm{M}+\mathrm{NH}_{4}\right]^{+}$calcd. 598.3555, found 598.3550.

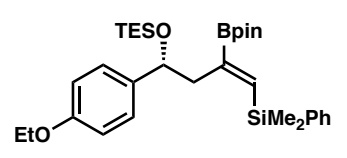

(R,Z)-((4-(Dimethyl(phenyl)silyl)-1-(4-ethoxyphenyl)-3-(4,4,5,5-t etramethyl-1,3,2-dioxaborolan-2-yl)but-3-en-1-yl)oxy)triethylsil

ane (3c) Prepared according to the general procedure. The crude mixture was purified by column chromatography with hexane and ethyl acetate to give 
the title compound as colorless oil in $84 \%$ yield $(48 \mathrm{mg}) .{ }^{1} \mathrm{H} \mathrm{NMR}\left(600 \mathrm{MHz}, \mathrm{CDCl}_{3}\right) \delta$ $7.46-7.47$ (m, 2H), $7.29-7.31$ (m, 3H), 7.11 (d, $J=8.6 \mathrm{~Hz}, 2 \mathrm{H}), 6.77$ (d, $J=8.5 \mathrm{~Hz}$, 2H), 6.64 (s, 1H), 4.83 (dd, $J=6.7,6.6 \mathrm{~Hz}, 1 \mathrm{H}), 3.99$ (q, $J=7.0 \mathrm{~Hz}, 2 \mathrm{H}), 2.66$ (dd, $J=$ 12.8, 7.4 Hz, 1H), 2.45 (dd, $J=12.9,6.0 \mathrm{~Hz}, 1 \mathrm{H}), 1.40$ (t, $J=7.0 \mathrm{~Hz}, 3 \mathrm{H}), 1.271(\mathrm{~s}, 6 \mathrm{H})$, $1.265(\mathrm{~s}, 6 \mathrm{H}), 0.83(\mathrm{t}, J=7.9 \mathrm{~Hz}, 9 \mathrm{H}), 0.39-0.50(\mathrm{~m}, 6 \mathrm{H}), 0.31(\mathrm{~s}, 3 \mathrm{H}), 0.30(\mathrm{~s}, 3 \mathrm{H}) .{ }^{13} \mathrm{C}$ NMR (151 MHz, $\left.\mathrm{CDCl}_{3}\right) \delta 158.0,146.8,139.9,138.3,134.2,129.0,128.0,127.4,113.9$, 83.7, 74.3, 63.6, 47.3, 25.4, 25.1, 15.3, 7.2, 5.1, -0.4, -0.5. HRMS $\left(\mathrm{ESI}^{+}\right): \mathrm{m} / \mathrm{z}$ for $\mathrm{C}_{32} \mathrm{H}_{51} \mathrm{BO}_{4} \mathrm{Si}_{2} \mathrm{Na}[\mathrm{M}+\mathrm{Na}]^{+}$calcd. 589.3317, found 589.3314 .

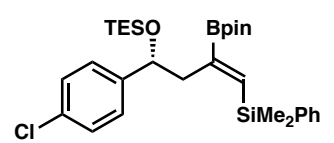

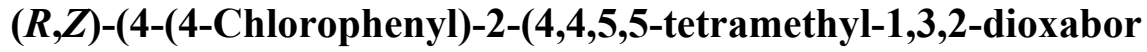
olan-2-yl)-4-((triethylsilyl)oxy)but-1-en-1-yl)dimethyl(phenyl)sila ne (3d) Prepared according to the general procedure. The crude mixture was purified by column chromatography with hexane and ethyl acetate to give the title compound as colorless oil in $83 \%$ yield $(46 \mathrm{mg}) .{ }^{1} \mathrm{H}$ NMR $\left(600 \mathrm{MHz}, \mathrm{CDCl}_{3}\right) \delta$ $7.43-7.45$ (m, 2H), $7.30-7.32$ (m, 3H), 7.20 (d, $J=8.3 \mathrm{~Hz}, 2 \mathrm{H}), 7.12$ (d, $J=8.2 \mathrm{~Hz}$, 2H), $6.67(\mathrm{~s}, 1 \mathrm{H}), 4.86(\mathrm{dd}, J=6.7,6.5 \mathrm{~Hz}, 2 \mathrm{H}), 2.64(\mathrm{dd}, J=12.8,7.2 \mathrm{~Hz}, 1 \mathrm{H}), 2.42(\mathrm{dd}$, $J=12.8,6.3 \mathrm{~Hz}, 1 \mathrm{H}), 1.27$ (s, 12H), $0.83(\mathrm{t}, J=7.9 \mathrm{~Hz}, 9 \mathrm{H}), 0.40-0.51$ (m, 6H), 0.30 (s, $3 \mathrm{H}), 0.28(\mathrm{~s}, 3 \mathrm{H}) .{ }^{13} \mathrm{C} \mathrm{NMR}\left(151 \mathrm{MHz}, \mathrm{CDCl}_{3}\right) \delta 147.5,144.7,139.6,134.2,132.5,129.1$, 128.2, 128.0, 127.7, 83.8, 74.0, 47.2, 25.4, 25.1, 7.2, 5.0, -0.5, -0.6. HRMS $\left(\mathrm{ESI}^{+}\right): \mathrm{m} / \mathrm{z}$ for $\mathrm{C}_{30} \mathrm{H}_{46} \mathrm{BClO}_{3} \mathrm{Si}_{2}[\mathrm{M}+\mathrm{Na}]^{+}$calcd. 579.2665, found 579.2672.

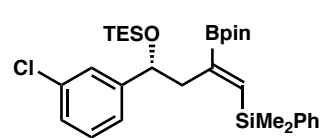

\section{(R,Z)-(4-(3-Chlorophenyl)-2-(4,4,5,5-tetramethyl-1,3,2-dioxaboro} lan-2-yl)-4-((triethylsilyl)oxy)but-1-en-1-yl)dimethyl(phenyl)silan

e (3e) Prepared according to the general procedure. The crude mixture was purified by column chromatography with hexane and ethyl acetate to give the title compound as colorless oil in $86 \%$ yield $(48 \mathrm{mg}) .{ }^{1} \mathrm{H} \mathrm{NMR}\left(600 \mathrm{MHz}, \mathrm{CDCl}_{3}\right) \delta$ $7.46-7.47(\mathrm{~m}, 2 \mathrm{H}), 7.30-7.33(\mathrm{~m}, 3 \mathrm{H}), 7.21(\mathrm{~s}, 1 \mathrm{H}), 7.14-7.18(\mathrm{~m}, 2 \mathrm{H}), 7.04-7.05$ (m, 1H), $6.69(\mathrm{~s}, 1 \mathrm{H}), 4.86(\mathrm{dd} J=6.7,6.6 \mathrm{~Hz}, 1 \mathrm{H}), 2.63(\mathrm{dd}, J=12.9,7.4 \mathrm{~Hz}, 1 \mathrm{H}), 2.43$ (dd, $J=12.9,6.0 \mathrm{~Hz}, 1 \mathrm{H}), 1.28(\mathrm{~s}, 12 \mathrm{H}), 0.84$ (t, $J=7.9 \mathrm{~Hz}, 9 \mathrm{H}), 0.40-0.52(\mathrm{~m}, 6 \mathrm{H})$, $0.31(\mathrm{~s}, 3 \mathrm{H}), 0.29$ (s, 3H). ${ }^{13} \mathrm{C}$ NMR $\left(151 \mathrm{MHz}, \mathrm{CDCl}_{3}\right) \delta 148.4,147.7,139.6,134.2$, 133.9, 129.4, 129.1, 128.0, 127.1, 126.4, 124.5, 83.8, 74.0, 47.1, 25.3, 25.1, 7.2, 5.0, -0.5, -0.6. HRMS $\left(\mathrm{ESI}^{+}\right): \mathrm{m} / \mathrm{z}$ for $\mathrm{C}_{30} \mathrm{H}_{50} \mathrm{BNClO}_{3} \mathrm{Si}_{2}\left[\mathrm{M}+\mathrm{NH}_{4}\right]^{+}$calcd. 574.3111, found 574.3112 .

$(R, Z)-(4-(2-C h l o r o p h e n y l)-2-(4,4,5,5-t e t r a m e t h y l-1,3,2-d i o x a b o r o l a n-2-y l)-4-((t r i e t h y$ lsilyl)oxy)but-1-en-1-yl)dimethyl(phenyl)silane (3f) Prepared according to the general 


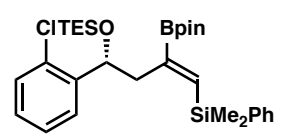

procedure. The crude mixture was purified by column chromatography with hexane and ethyl acetate to give the title compound as colorless oil in $92 \%$ yield $(51 \mathrm{mg}) .{ }^{1} \mathrm{H}$ NMR $\left(600 \mathrm{MHz}, \mathrm{CDCl}_{3}\right) \delta 7.48-7.51(\mathrm{~m}$, $3 \mathrm{H}), 7.28-7.31(\mathrm{~m}, 3 \mathrm{H}), 7.21-7.24(\mathrm{~m}, 2 \mathrm{H}), 7.12$ (ddd, $J=7.7,7.4,1.6 \mathrm{~Hz}, 1 \mathrm{H}), 6.68$ (s, 1H), $5.41(\mathrm{dd}, J=7.7,5.1 \mathrm{~Hz}, 1 \mathrm{H}), 2.56(\mathrm{dd}, J=13.0,8.1 \mathrm{~Hz}, 1 \mathrm{H}), 2.52$ (dd, $J=$ 13.1, $5.0 \mathrm{~Hz}, 1 \mathrm{H}), 1.29$ (s, 6H), $1.28(\mathrm{~s}, 6 \mathrm{H}), 0.83(\mathrm{t}, J=7.9 \mathrm{~Hz}, 9 \mathrm{H}), 0.53-0.41(\mathrm{~m}, 6 \mathrm{H})$, 0.35 (s, 3H), 0.33 (s, 3H). ${ }^{13} \mathrm{C}$ NMR $\left(151 \mathrm{MHz}, \mathrm{CDCl}_{3}\right) \delta 147.1,144.2,139.9,134.2$, 131.5, 129.1, 129.0, 128.6, 128.1, 128.0, 127.1, 83.8, 70.5, 46.0, 25.4, 25.2, 7.1, 4.9, -0.3, -0.4. HRMS $\left(\mathrm{ESI}^{+}\right): \mathrm{m} / \mathrm{z}$ for $\mathrm{C}_{30} \mathrm{H}_{50} \mathrm{BNClO}_{3} \mathrm{Si}_{2}\left[\mathrm{M}+\mathrm{NH}_{4}\right]^{+}$calcd. 574.3111, found 574.3099 .

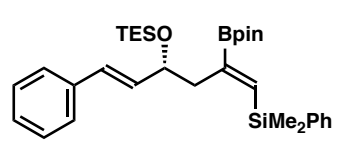

(((R,1E,5Z)-6-(Dimethyl(phenyl)silyl)-1-phenyl-5-(4,4,5,5-tetram ethyl-1,3,2-dioxaborolan-2-yl)hexa-1,5-dien-3-yl)oxy)triethylsila

ne (3g) Prepared according to the general procedure. The crude mixture was purified by column chromatography with hexane and ethyl acetate to give the title compound as colorless oil in $86 \%$ yield $(47 \mathrm{mg}) .{ }^{1} \mathrm{H}$ NMR $\left(600 \mathrm{MHz}, \mathrm{CDCl}_{3}\right) \delta$ $7.51(\mathrm{~d}, J=6.4 \mathrm{~Hz}, 2 \mathrm{H}), 7.27-7.34(\mathrm{~m}, 7 \mathrm{H}), 7.19-7.22(\mathrm{~m}, 1 \mathrm{H}), 6.72(\mathrm{~s}, 1 \mathrm{H}), 6.34(\mathrm{~d}, J$ $=15.9 \mathrm{~Hz}, 1 \mathrm{H}), 6.05(\mathrm{dd}, J=15.9,6.6 \mathrm{~Hz}, 1 \mathrm{H}), 4.50(\mathrm{ddd}, J=6.9,6.8,6.8 \mathrm{~Hz}, 1 \mathrm{H}), 2.60$ (dd, $J=12.7,6.0 \mathrm{~Hz}, 1 \mathrm{H}), 2.49$ (dd, $J=12.6,8.2 \mathrm{~Hz}, 1 \mathrm{H}), 1.24(\mathrm{~s}, 6 \mathrm{H}), 1.23(\mathrm{~s}, 6 \mathrm{H}), 0.93$ (t, $J=7.9 \mathrm{~Hz}, 9 \mathrm{H}), 0.58$ (q, $J=7.9 \mathrm{~Hz}, 6 \mathrm{H}), 0.39$ (s, 6H). ${ }^{13} \mathrm{C} \mathrm{NMR}\left(151 \mathrm{MHz}, \mathrm{CDCl}_{3}\right) \delta$ 147.2, 139.6, 137.4, 134.2, 133.5, 129.1, 129.0, 128.7, 128.0, 127.5, 126.8, 83.8, 73.4, 44.8, 25.3, 25.1, 7.3, 5.2, -0.37, -0.40. HRMS $\left(\mathrm{ESI}^{+}\right): \mathrm{m} / \mathrm{z}$ for $\mathrm{C}_{32} \mathrm{H}_{49} \mathrm{BO}_{3} \mathrm{Si}_{2} \mathrm{Na}[\mathrm{M}+\mathrm{Na}]^{+}$ calcd. 571.3211, found 571.3185.

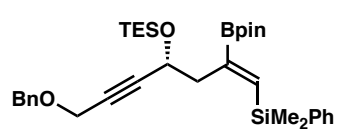

$(R, Z)-((7-(B e n z y l o x y)-1-(d i m e t h y l(p h e n y l) s i l y l)-2-(4,4,5,5-t e t r a$ methyl-1,3,2-dioxaborolan-2-yl)hept-1-en-5-yn-4-yl)oxy)triethyl

silane (3h) Prepared according to the general procedure. The crude mixture was purified by column chromatography with hexane and ethyl acetate to give the title compound as colorless oil in $91 \%$ yield $(54 \mathrm{mg}) .{ }^{1} \mathrm{H}$ NMR $\left(600 \mathrm{MHz}, \mathrm{CDCl}_{3}\right) \delta$ $7.53-7.54(\mathrm{~m}, 2 \mathrm{H}), 7.28-7.34(\mathrm{~m}, 8 \mathrm{H}), 6.79(\mathrm{~s}, 1 \mathrm{H}), 4.78(\mathrm{dd}, J=7.3,7.2 \mathrm{~Hz}, 1 \mathrm{H})$, 4.55 (s, 2H), 4.13 (s, 2H), 2.66 (dd, $J=12.8,7.4 \mathrm{~Hz}, 1 \mathrm{H}), 2.61$ (dd, $J=12.9,7.4 \mathrm{~Hz}, 1 \mathrm{H}$ ), $1.26(\mathrm{~s}, 12 \mathrm{H}), 0.95(\mathrm{t}, J=7.9 \mathrm{~Hz}, 9 \mathrm{H}), 0.58-0.67(\mathrm{~m}, 6 \mathrm{H}), 0.44(\mathrm{~s}, 6 \mathrm{H}) .{ }^{13} \mathrm{C}$ NMR $(151$ $\left.\mathrm{MHz}, \mathrm{CDCl}_{3}\right) \delta 148.3,139.6,137.8,134.2,129.1,128.7,128.5,128.1,128.0,88.9,83.9$, $80.2,71.5,62.5,57.8,44.7,25.3,25.0,7.2,5.0,-0.2,-0.3$. HRMS $\left(\mathrm{ESI}^{+}\right): \mathrm{m} / \mathrm{z}$ for $\mathrm{C}_{34} \mathrm{H}_{55} \mathrm{BNO}_{4} \mathrm{Si}_{2}\left[\mathrm{M}+\mathrm{NH}_{4}\right]^{+}$calcd. 608.3763 , found 608.3743 . 


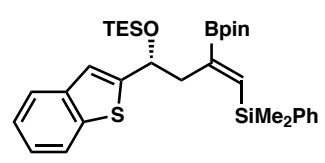

(R,Z)-(4-(Benzo[b]thiophen-2-yl)-2-(4,4,5,5-tetramethyl-1,3,2-dio xaborolan-2-yl)-4-((triethylsilyl)oxy)but-1-en-1-yl)dimethyl(phen

yl)silane (3i) Prepared according to the general procedure. The crude mixture was purified by column chromatography with hexane and ethyl acetate to give the title compound as colorless oil in $88 \%$ yield $(51 \mathrm{mg}) .{ }^{1} \mathrm{H} \mathrm{NMR}\left(600 \mathrm{MHz}, \mathrm{CDCl}_{3}\right) \delta$ $7.77(\mathrm{~d}, J=7.9 \mathrm{~Hz}, 1 \mathrm{H}), 7.65(\mathrm{~d}, J=7.8 \mathrm{~Hz}, 1 \mathrm{H}), 7.42-7.43(\mathrm{~m}, 2 \mathrm{H}), 7.22-7.31(\mathrm{~m}$, $5 \mathrm{H}), 6.96(\mathrm{~s}, 1 \mathrm{H}), 6.75(\mathrm{~s}, 1 \mathrm{H}), 5.31(\mathrm{dd}, J=6.8,6.8 \mathrm{~Hz}, 1 \mathrm{H}), 2.82(\mathrm{dd}, J=13.0,6.9 \mathrm{~Hz}$, $1 \mathrm{H}), 2.66(\mathrm{dd}, J=13.0,6.9 \mathrm{~Hz}, 1 \mathrm{H}), 1.26(\mathrm{~s}, 12 \mathrm{H}), 0.90(\mathrm{t}, J=8.0 \mathrm{~Hz}, 9 \mathrm{H}), 0.51-0.61$ (m, 6H), 0.31 (s, 3H), 0.30 (s, 3H). ${ }^{13} \mathrm{C}$ NMR (151 MHz, $\left.\mathrm{CDCl}_{3}\right) \delta$ 151.7, 148.4, 140.0, 139.5, 139.4, 134.1, 129.0, 128.0, 124.1, 123.7, 123.5, 122.7, 118.9, 83.9, 71.2, 47.2, 25.4, 25.1, 7.2, 5.1, -0.5, -0.7. HRMS $\left(\mathrm{ESI}^{+}\right): \mathrm{m} / \mathrm{z}$ for $\mathrm{C}_{32} \mathrm{H}_{47} \mathrm{BO}_{3} \mathrm{SSi}_{2} \mathrm{Na}[\mathrm{M}+\mathrm{Na}]^{+}$calcd. 601.2775 , found 601.2764 .

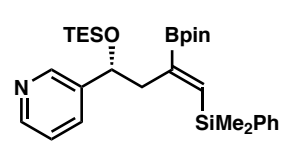

(R,Z)-3-(4-(Dimethyl(phenyl)silyl)-3-(4,4,5,5-tetramethyl-1,3,2-diox aborolan-2-yl)-1-((triethylsilyl)oxy)but-3-en-1-yl)pyridine

Prepared according to the general procedure. The crude mixture was purified by column chromatography with hexane and ethyl acetate to give the title compound as colorless oil in 92\% yield (48 mg). ${ }^{1} \mathrm{H}$ NMR $\left(600 \mathrm{MHz}, \mathrm{CDCl}_{3}\right) \delta 8.42-$ $8.43(\mathrm{~m}, 2 \mathrm{H}), 7.52$ (d, $J=7.8 \mathrm{~Hz}, 1 \mathrm{H}), 7.44-7.46(\mathrm{~m}, 2 \mathrm{H}), 7.30-7.34(\mathrm{~m}, 3 \mathrm{H}), 7.17$ (dd, $J=7.6,4.9 \mathrm{~Hz}, 1 \mathrm{H}), 6.71(\mathrm{~s}, 1 \mathrm{H}), 4.96(\mathrm{dd}, J=6.8,6.7 \mathrm{~Hz}, 1 \mathrm{H}), 2.69$ (dd, $J=13.0,7.2$ $\mathrm{Hz}, 1 \mathrm{H}), 2.46$ (dd, $J=13.0,6.4 \mathrm{~Hz}, 1 \mathrm{H}), 1.28$ (s, 6H), 1.27 (s, 6H), 0.83 (t, $J=7.9 \mathrm{~Hz}$, 9H), $0.41-0.52(\mathrm{~m}, 6 \mathrm{H}), 0.28(\mathrm{~s}, 3 \mathrm{H}), 0.26(\mathrm{~s}, 3 \mathrm{H}) .{ }^{13} \mathrm{C} \mathrm{NMR}\left(151 \mathrm{MHz}, \mathrm{CDCl}_{3}\right) \delta 148.6$, 148.2 (2 C signals), 141.3, 139.5, 134.2, 134.0, 129.1, 128.1, 123.4, 83.9, 72.4, 47.1, 25.4, 25.1, 7.2, 5.0, $-0.57,-0.62$. HRMS $\left(\mathrm{ESI}^{+}\right): \mathrm{m} / \mathrm{z}$ for $\mathrm{C}_{29} \mathrm{H}_{47} \mathrm{BNO}_{3} \mathrm{Si}_{2}[\mathrm{M}+\mathrm{H}]^{+}$calcd. 524.3188 , found 524.3185 .

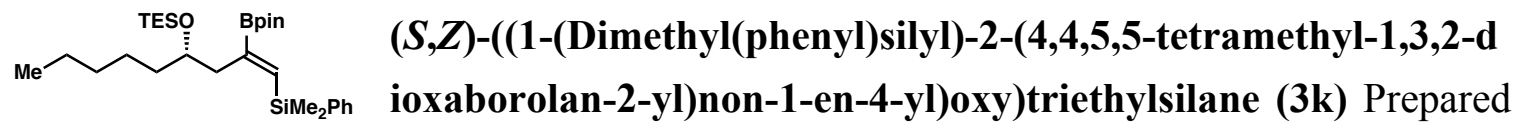

according to the general procedure. The crude mixture was purified by column chromatography with hexane and ethyl acetate to give the title compound as colorless oil in $72 \%$ yield $(37 \mathrm{mg}) .{ }^{1} \mathrm{H}$ NMR ( $\left.600 \mathrm{MHz}, \mathrm{CDCl}_{3}\right) \delta 7.52-7.54(\mathrm{~m}, 2 \mathrm{H}), 7.32-7.33(\mathrm{~m}$, $3 \mathrm{H}), 6.64(\mathrm{~s}, 1 \mathrm{H}), 3.89-3.92(\mathrm{~m}, 1 \mathrm{H}), 2.44(\mathrm{dd}, J=12.9,4.6 \mathrm{~Hz}, 1 \mathrm{H}), 2.31$ (dd, $J=12.8$, $8.5 \mathrm{~Hz}, 1 \mathrm{H}), 1.11-1.36(\mathrm{~m}, 20 \mathrm{H}), 0.92(\mathrm{t}, J=8.0 \mathrm{~Hz}, 9 \mathrm{H}), 0.85$ (t, $J=7.2 \mathrm{~Hz}, 3 \mathrm{H}), 0.55$ -0.59 (m, 6H), $0.42(\mathrm{~s}, 3 \mathrm{H}), 0.41(\mathrm{~s}, 3 \mathrm{H}) .{ }^{13} \mathrm{C}$ NMR $\left(151 \mathrm{MHz}, \mathrm{CDCl}_{3}\right) \delta$ 146.0, 139.7, 134.2, 129.1, 128.0, 83.8, 72.0, 45.0, 37.3, 32.2, 25.9, 25.3, 25.0, 23.0, 14.5, 7.3, 5.3, -0.3, -0.4. HRMS $\left(\mathrm{ESI}^{+}\right): \mathrm{m} / \mathrm{z}$ for $\mathrm{C}_{29} \mathrm{H}_{54} \mathrm{BO}_{3} \mathrm{Si}_{2}[\mathrm{M}+\mathrm{H}]^{+}$calcd. 517.3705, found 517.3688. 


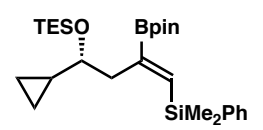

(R,Z)-(4-Cyclopropyl-2-(4,4,5,5-tetramethyl-1,3,2-dioxaborolan-2-yl) -4-((triethylsilyl)oxy)but-1-en-1-yl)dimethyl(phenyl)silane

Prepared according to the general procedure. The crude mixture was purified by column chromatography with hexane and ethyl acetate to give the title compound as colorless oil in $82 \%$ yield $(40 \mathrm{mg}) .{ }^{1} \mathrm{H}$ NMR $\left(600 \mathrm{MHz}, \mathrm{CDCl}_{3}\right) \delta 7.53-$ $7.54(\mathrm{~m}, 2 \mathrm{H}), 7.32-7.33(\mathrm{~m}, 3 \mathrm{H}), 6.64(\mathrm{~s}, 1 \mathrm{H}), 3.37$ (ddd, $J=7.0,7.0,6.9 \mathrm{~Hz}, 1 \mathrm{H}), 2.52$ (d, $J=6.8 \mathrm{~Hz}, 2 \mathrm{H}), 1.25(\mathrm{~s}, 12 \mathrm{H}), 0.91(\mathrm{t}, J=7.9 \mathrm{~Hz}, 9 \mathrm{H}), 0.68-0.73(\mathrm{~m}, 1 \mathrm{H}), 0.55(\mathrm{q}, J$ $=7.9 \mathrm{~Hz}, 6 \mathrm{H}), 0.424(\mathrm{~s}, 3 \mathrm{H}), 0.417(\mathrm{~s}, 3 \mathrm{H}), 0.28-0.33(\mathrm{~m}, 2 \mathrm{H}), 0.15-0.19(\mathrm{~m}, 1 \mathrm{H})$, $0.09-0.12(\mathrm{~m}, 1 \mathrm{H}) .{ }^{13} \mathrm{C}$ NMR $\left(151 \mathrm{MHz}, \mathrm{CDCl}_{3}\right) \delta 145.6,139.8,134.2,129.1,128.0$, 83.7, 75.5, 45.6, 25.2, 25.1, 17.7, 7.3, 5.3, 3.5, 3.0, -0.27, -0.31. HRMS (ESI $\left.{ }^{+}\right): \mathrm{m} / \mathrm{z}$ for $\mathrm{C}_{27} \mathrm{H}_{47} \mathrm{BO}_{3} \mathrm{Si}_{2} \mathrm{Na}[\mathrm{M}+\mathrm{Na}]^{+}$calcd. 509.3055, found 509.3073.

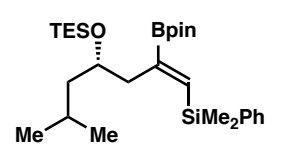

(S,Z)-((1-(Dimethyl(phenyl)silyl)-6-methyl-2-(4,4,5,5-tetramethyl-1, 3,2-dioxaborolan-2-yl)hept-1-en-4-yl)oxy)triethylsilane

(3m)

Prepared according to the general procedure. The crude mixture was purified by column chromatography with hexane and ethyl acetate to give compound $\mathbf{3 m}$ in $74 \%$ yield $(37 \mathrm{mg}) .{ }^{1} \mathrm{H}$ NMR (600 MHz, $\left.\mathrm{CDCl}_{3}\right) \delta 7.52-7.54(\mathrm{~m}, 2 \mathrm{H}), 7.32-7.33(\mathrm{~m}$, $3 \mathrm{H}), 6.64$ (s, 1H), $4.01-4.05$ (m, 1H), 2.45 (dd, $J=12.3,4.3 \mathrm{~Hz}, 1 \mathrm{H}), 2.30$ (dd, $J=12.7$, $9.0 \mathrm{~Hz}, 1 \mathrm{H}), 1.65-1.71(\mathrm{~m}, 1 \mathrm{H}), 1.26(\mathrm{~s}, 12 \mathrm{H}), 1.06-1.11(\mathrm{~m}, 1 \mathrm{H}), 0.98-1.02(\mathrm{~m}, 1 \mathrm{H})$, $0.92(\mathrm{t}, J=7.9 \mathrm{~Hz}, 9 \mathrm{H}), 0.81(\mathrm{~d}, J=6.6 \mathrm{~Hz}, 3 \mathrm{H}), 0.77(\mathrm{~d}, J=6.5 \mathrm{~Hz}, 3 \mathrm{H}), 0.53-0.63(\mathrm{~m}$, $6 \mathrm{H}), 0.412$ (s, 3H), 0.407 (s, 3H). ${ }^{13} \mathrm{C}$ NMR $\left(151 \mathrm{MHz}, \mathrm{CDCl}_{3}\right) \delta$ 146.2, 139.6, 134.2, 129.1, 128.0, 83.8, 69.8, 46.7, 45.6, 25.3, 25.1, 24.5, 24.0, 22.1, 7.3, 5.4, -0.3, -0.4. HRMS ( $\left.\mathrm{ESI}^{+}\right): \mathrm{m} / \mathrm{z}$ for $\mathrm{C}_{28} \mathrm{H}_{51} \mathrm{BO}_{3} \mathrm{Si}_{2} \mathrm{Na}[\mathrm{M}+\mathrm{Na}]^{+}$calcd. 525.3368, found 525.3356.

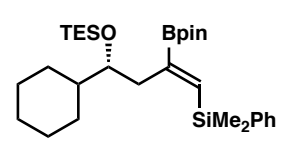

(R,Z)-(4-Cyclohexyl-2-(4,4,5,5-tetramethyl-1,3,2-dioxaborolan-2-yl) -4-((triethylsilyl)oxy)but-1-en-1-yl)dimethyl(phenyl)silane

(3n)

Prepared according to the general procedure. The crude mixture was

purified by column chromatography with hexane and ethyl acetate to give the title compound as colorless oil in $85 \%$ yield $(45 \mathrm{mg}) .{ }^{1} \mathrm{H}$ NMR $\left(600 \mathrm{MHz}, \mathrm{CDCl}_{3}\right) \delta 7.53-$ $7.55(\mathrm{~m}, 2 \mathrm{H}), 7.32-7.33(\mathrm{~m}, 3 \mathrm{H}), 6.61(\mathrm{~s}, 1 \mathrm{H}), 3.84-3.87(\mathrm{~m}, 1 \mathrm{H}), 2.31-2.37(\mathrm{~m}, 2 \mathrm{H})$, $1.48-1.69(\mathrm{~m}, 6 \mathrm{H}), 1.26(\mathrm{~s}, 12 \mathrm{H}), 1.22-1.25(\mathrm{~m}, 1 \mathrm{H}), 0.97-1.15(\mathrm{~m}, 4 \mathrm{H}), 0.92(\mathrm{t}, J=$ $8.0 \mathrm{~Hz}, 9 \mathrm{H}), 0.55$ (q, $J=7.8 \mathrm{~Hz}, 6 \mathrm{H}), 0.43(\mathrm{~s}, 3 \mathrm{H}), 0.40(\mathrm{~s}, 3 \mathrm{H}) .{ }^{13} \mathrm{C}$ NMR $(151 \mathrm{MHz}$, $\left.\mathrm{CDCl}_{3}\right) \delta 145.4,139.9,134.2,129.0,128.0,83.7,75.6,44.1,40.8,29.4,27.5,27.1,27.0$, 26.8, 25.3, 25.1, 7.4, 5.5, -0.38, -0.43. HRMS $\left(\mathrm{ESI}^{+}\right): \mathrm{m} / \mathrm{z}$ for $\mathrm{C}_{30} \mathrm{H}_{54} \mathrm{BO}_{3} \mathrm{Si}_{2}[\mathrm{M}+\mathrm{H}]^{+}$ calcd. 529.3705, found 529.3708. 


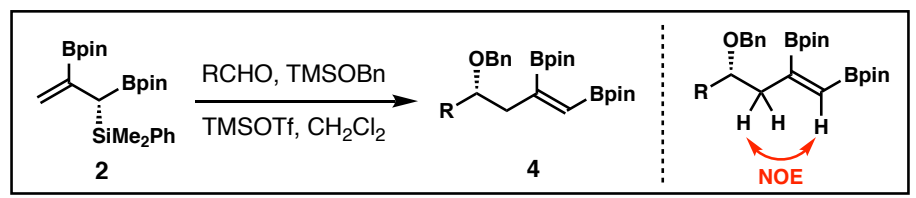

General procedure for the syntheses of benzyl ethers 4 using aldehyde allylsilation with reagent 2: To a reaction flask containing a Teflon-coated magnetic stirring bar were sequentially added freshly distilled aldehydes ( $0.1 \mathrm{mmol}, 1.0$ equiv, if it is a liquid), benzyl trimethylsilyl ether (TMSOBn, $0.13 \mathrm{mmol}, 1.3$ equiv) and $\mathrm{CH}_{2} \mathrm{Cl}_{2}$ (1.0 mL). After stirring at $-78{ }^{\circ} \mathrm{C}$ for $5 \mathrm{~min}$, trimethylsilyl trifluoromethanesulfonate (TMSOTf, 0.1 mmol, 1.0 equiv) was added to the flask and stirred for $6 \mathrm{~h}$ at $-78^{\circ} \mathrm{C}$. Then a solution of reagent 2 (0.12 mmol, 1.2 equiv) in $\mathrm{CH}_{2} \mathrm{Cl}_{2}(0.2 \mathrm{~mL})$ was added, and the mixture was kept stirring at $-78{ }^{\circ} \mathrm{C}$ for additional $12 \mathrm{~h}$. Then water $(0.5 \mathrm{~mL})$ was added at $-78{ }^{\circ} \mathrm{C}$, and the reaction mixture was warmed to ambient temperature. The organic layer was separated and the aqueous layer was extracted with $\mathrm{Et}_{2} \mathrm{O}(3 \times 1 \mathrm{~mL})$. The combined organic layers were dried over anhydrous sodium sulfate, filtered, and concentrated under reduced pressure. The $E / Z$ ratio was determined by ${ }^{1} \mathrm{H}$ NMR analysis of the crude reaction mixture. Purification of the crude product was performed by flash column chromatography (gradient elution with hexane and ethyl acetate) to give product 4 . The olefin geometry of $\mathbf{4}$ was assigned as $E$ by NOE studies.

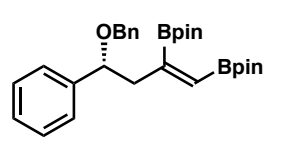

$(R, E)-2,2 '-(4-(B e n z y l o x y)-4-p h e n y l b u t-1-e n e-1,2-d i y l) b i s(4,4,5,5-t e t$

ramethyl-1,3,2-dioxaborolane) (4a) Prepared according to the general procedure. The crude mixture was purified by column chromatography with hexane and ethyl acetate to give the title compound as colorless oil in $82 \%$ yield (40 $\mathrm{mg}, E / Z=11 / 1) .{ }^{1} \mathrm{H} \mathrm{NMR}\left(600 \mathrm{MHz}, \mathrm{CDCl}_{3}\right) \delta 7.22-7.33(\mathrm{~m}, 10 \mathrm{H}), 5.89(\mathrm{~s}, 1 \mathrm{H}), 4.47$ $(\mathrm{dd}, J=8.0,4.9 \mathrm{~Hz}, 1 \mathrm{H}), 4.42\left(\mathrm{~d}, J_{A-B}=12.1 \mathrm{~Hz}, 1 \mathrm{H}\right), 4.28\left(\mathrm{~d}, J_{A-B}=12.1 \mathrm{~Hz}, 1 \mathrm{H}\right), 2.79$ (dd, $J=13.3,8.6 \mathrm{~Hz}, 1 \mathrm{H}), 2.54(\mathrm{dd}, J=13.8,3.9 \mathrm{~Hz}, 1 \mathrm{H}), 1.27$ (s, 12H), 1.23 (s, 6H), $1.21(\mathrm{~s}, 6 \mathrm{H}) .{ }^{13} \mathrm{C}$ NMR $\left(151 \mathrm{MHz}, \mathrm{CDCl}_{3}\right) \delta 142.7,139.1,128.6,128.5,128.0,127.7$, 127.5, 127.2, 83.9, 83.6, 81.1, 70.5, 49.2, 25.21, 25.17, 25.15. HRMS $\left(\mathrm{ESI}^{+}\right): \mathrm{m} / \mathrm{z}$ for $\mathrm{C}_{29} \mathrm{H}_{40} \mathrm{~B}_{2} \mathrm{O}_{5} \mathrm{Na}[\mathrm{M}+\mathrm{Na}]^{+}$calcd. 513.2960, found 513.2968.

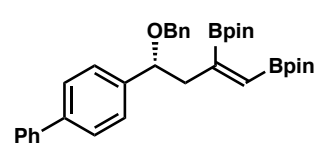

(R,E)-2,2'-(4-([1,1'-Biphenyl]-4-yl)-4-(benzyloxy)but-1-ene-1,2-di yl)bis(4,4,5,5-tetramethyl-1,3,2-dioxaborolane) (4b) Prepared according to the general procedure. The crude mixture was purified by column chromatography with hexane and ethyl acetate to give the title compound as colorless oil in $76 \%$ yield (43 mg, $E / Z=8 / 1) .{ }^{1} \mathrm{H}$ NMR $\left(400 \mathrm{MHz}, \mathrm{CDCl}_{3}\right) \delta 7.55-7.61$ 
(m, 4H), $7.39-7.45(\mathrm{~m}, 4 \mathrm{H}), 7.22-7.35(\mathrm{~m}, 6 \mathrm{H}), 5.93(\mathrm{~s}, 1 \mathrm{H}), 4.53(\mathrm{dd}, J=7.8,5.3 \mathrm{~Hz}$, $1 \mathrm{H}), 4.47\left(\mathrm{~d}, J_{A-B}=12.1 \mathrm{~Hz}, 1 \mathrm{H}\right), 4.33\left(\mathrm{~d}, J_{A-B}=12.1 \mathrm{~Hz}, 1 \mathrm{H}\right), 2.84$ (ddd, $J=13.8,7.9$, $1.2 \mathrm{~Hz}, 1 \mathrm{H}), 2.60$ (ddd, $J=13.7,5.2,1.1 \mathrm{~Hz}, 1 \mathrm{H}), 1.27(\mathrm{~s}, 12 \mathrm{H}), 1.24(\mathrm{~s}, 6 \mathrm{H}), 1.22$ (s, $6 \mathrm{H}) .{ }^{13} \mathrm{C}$ NMR $\left(151 \mathrm{MHz}, \mathrm{CDCl}_{3}\right) \delta 141.7,141.3,140.6,139.0,133.4,129.0,128.5$, 128.0, 127.7, 127.53, 127.45, 127.41 (2 C signals), 83.9, 83.6, 80.9, 70.5, 49.0, 25.20, 25.17. HRMS (ESI $\left.{ }^{+}\right): \mathrm{m} / \mathrm{z}$ for $\mathrm{C}_{35} \mathrm{H}_{48} \mathrm{~B}_{2} \mathrm{NO}_{5}\left[\mathrm{M}+\mathrm{NH}_{4}\right]^{+}$calcd. 584.3719, found 584.3691.

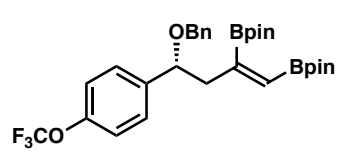

(R,E)-2,2'-(4-(Benzyloxy)-4-(4-(trifluoromethoxy)phenyl)but-1-e ne-1,2-diyl)bis(4,4,5,5-tetramethyl-1,3,2-dioxaborolane)

(4c)

Prepared according to the general procedure. The crude mixture was purified by column chromatography with hexane and ethyl acetate to give the title compound as colorless oil in $87 \%$ yield $(50 \mathrm{mg}, E / Z=12 / 1) .{ }^{1} \mathrm{H} \mathrm{NMR}\left(600 \mathrm{MHz}, \mathrm{CDCl}_{3}\right)$ $\delta 7.34(\mathrm{~d}, J=8.3 \mathrm{~Hz}, 2 \mathrm{H}), 7.29-7.30(\mathrm{~m}, 4 \mathrm{H}), 7.23-7.26(\mathrm{~m}, 1 \mathrm{H}), 7.16(\mathrm{~d}, J=8.0 \mathrm{~Hz}$, 2H), $5.85(\mathrm{~s}, 1 \mathrm{H}), 4.47(\mathrm{dd}, J=6.7,6.2 \mathrm{~Hz}, 1 \mathrm{H}), 4.42\left(\mathrm{~d}, J_{A-B}=12.1 \mathrm{~Hz}, 1 \mathrm{H}\right), 4.27$ (d, $\left.J_{A-B}=12.1 \mathrm{~Hz}, 1 \mathrm{H}\right), 2.78(\mathrm{dd}, J=13.5,7.5 \mathrm{~Hz}, 1 \mathrm{H}), 2.52(\mathrm{dd}, J=13.6,5.5 \mathrm{~Hz}, 1 \mathrm{H}), 1.26$ $(\mathrm{s}, 12 \mathrm{H}), 1.22(\mathrm{~s}, 6 \mathrm{H}), 1.20(\mathrm{~s}, 6 \mathrm{H}) .{ }^{13} \mathrm{C} \mathrm{NMR}\left(151 \mathrm{MHz}, \mathrm{CDCl}_{3}\right) \delta 151.4,148.7,141.3$, 138.7, 133.9, 128.6, 128.5, 128.0, 127.7, 121.1, 120.7 (q, $J_{C-F}=257 \mathrm{~Hz}$ ), 84.0, 83.7, 80.3, 70.6, 48.9, 25.18, 25.16, 25.14. HRMS $\left(\mathrm{ESI}^{+}\right)$: $\mathrm{m} / \mathrm{z}$ for $\mathrm{C}_{30} \mathrm{H}_{43} \mathrm{~B}_{2} \mathrm{~F}_{3} \mathrm{NO}_{6}\left[\mathrm{M}+\mathrm{NH}_{4}\right]^{+}$calcd. 592.3229 , found 592.3226 .

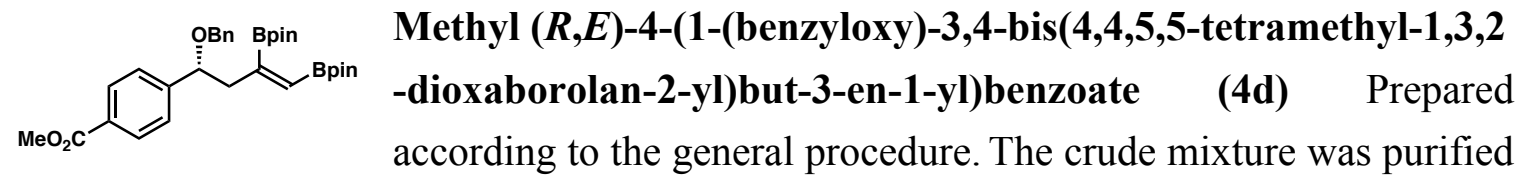

by column chromatography with hexane and ethyl acetate to give the title compound as colorless oil in $84 \%$ yield $(46 \mathrm{mg}, E / Z=15 / 1) .{ }^{1} \mathrm{H} \mathrm{NMR}\left(600 \mathrm{MHz}, \mathrm{CDCl}_{3}\right) \delta 8.00(\mathrm{~d}, J=$ $8.1 \mathrm{~Hz}, 2 \mathrm{H}), 7.40(\mathrm{~d}, J=8.1 \mathrm{~Hz}, 2 \mathrm{H}), 7.29-7.30(\mathrm{~m}, 4 \mathrm{H}), 7.23-7.26(\mathrm{~m}, 1 \mathrm{H}), 5.84$ (s, $1 \mathrm{H}), 4.52(\mathrm{dd}, J=7.1,5.9 \mathrm{~Hz}, 1 \mathrm{H}), 4.42\left(\mathrm{~d}, J_{A-B}=12.0 \mathrm{~Hz}, 1 \mathrm{H}\right), 4.27\left(\mathrm{~d}, J_{A-B}=12.0 \mathrm{~Hz}\right.$, $1 \mathrm{H}), 3.91(\mathrm{~s}, 3 \mathrm{H}), 2.78(\mathrm{dd}, J=13.5,7.6 \mathrm{~Hz}, 1 \mathrm{H}), 2.53(\mathrm{dd}, J=13.6,5.1 \mathrm{~Hz}, 1 \mathrm{H}), 1.26$ (s, 12H), $1.23(\mathrm{~s}, 6 \mathrm{H}), 1.21(\mathrm{~s}, 6 \mathrm{H}) .{ }^{13} \mathrm{C} \mathrm{NMR}\left(151 \mathrm{MHz}, \mathrm{CDCl}_{3}\right) \delta 167.4,148.0,138.6$, 133.9, 130.0, 129.5, 128.5, 128.0, 127.7, 127.2, 84.0, 83.7, 80.6, 70.8, 52.5, 48.9, 25.19, 25.15. HRMS (ESI $\left.{ }^{+}\right): \mathrm{m} / \mathrm{z}$ for $\mathrm{C}_{31} \mathrm{H}_{46} \mathrm{~B}_{2} \mathrm{NO}_{7}\left[\mathrm{M}+\mathrm{NH}_{4}\right]^{+}$calcd. 566.3460, found 566.3478 .

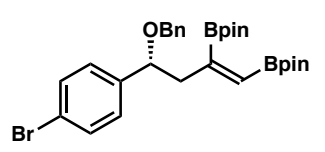

(R,E)-2,2'-(4-(Benzyloxy)-4-(4-bromophenyl)but-1-ene-1,2-diyl)bi s(4,4,5,5-tetramethyl-1,3,2-dioxaborolane) (4e) Prepared according to the general procedure. The crude mixture was purified by column 
chromatography with hexane and ethyl acetate to give the title compound as colorless oil in $88 \%$ yield $(50 \mathrm{mg}, E / Z=11 / 1) .{ }^{1} \mathrm{H}$ NMR $\left(600 \mathrm{MHz}, \mathrm{CDCl}_{3}\right) \delta 7.44(\mathrm{~d}, J=8.1 \mathrm{~Hz}, 2 \mathrm{H})$, $7.24-7.29(\mathrm{~m}, 5 \mathrm{H}), 7.20$ (d, $J=8.1 \mathrm{~Hz}, 2 \mathrm{H}), 5.84(\mathrm{~s}, 1 \mathrm{H}), 4.39-4.43(\mathrm{~m}, 2 \mathrm{H}), 4.25(\mathrm{~d}$, $\left.J_{A-B}=12.1 \mathrm{~Hz}, 1 \mathrm{H}\right), 2.77(\mathrm{dd}, J=13.5,7.4 \mathrm{~Hz}, 1 \mathrm{H}), 2.50(\mathrm{dd}, J=13.6,5.6 \mathrm{~Hz}, 1 \mathrm{H}), 1.26$ (s, 12H), $1.22(\mathrm{~s}, 6 \mathrm{H}), 1.20(\mathrm{~s}, 6 \mathrm{H}) \cdot{ }^{13} \mathrm{C}$ NMR $\left(151 \mathrm{MHz}, \mathrm{CDCl}_{3}\right) \delta 141.5,138.7,133.9$, 131.7, 129.1, 128.5, 128.0, 127.7, 121.5, 84.0, 83.6, 80.4, 70.5, 48.9, 25.18, 25.17, 25.15. HRMS (ESI $\left.{ }^{+}\right): \mathrm{m} / \mathrm{z}$ for $\mathrm{C}_{29} \mathrm{H}_{43} \mathrm{~B}_{2} \mathrm{BrNO}_{5}\left[\mathrm{M}+\mathrm{NH}_{4}\right]^{+}$calcd. 586.2511, found 586.2506.

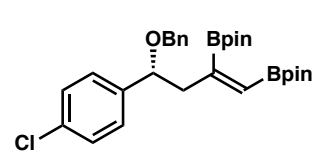

(R,E)-2,2'-(4-(Benzyloxy)-4-(4-chlorophenyl)but-1-ene-1,2-diyl)bi s(4,4,5,5-tetramethyl-1,3,2-dioxaborolane) (4f) Prepared according to the general procedure. The crude mixture was purified by column chromatography with hexane and ethyl acetate to give the title compound as colorless oil in $84 \%$ yield (44 mg, $E / Z=12 / 1) .{ }^{1} \mathrm{H}$ NMR $\left(600 \mathrm{MHz}, \mathrm{CDCl}_{3}\right) \delta 7.25-7.30(\mathrm{~m}, 9 \mathrm{H})$, $5.83(\mathrm{~s}, 1 \mathrm{H}), 4.43(\mathrm{dd}, J=8.6,6.5 \mathrm{~Hz}, 1 \mathrm{H}), 4.40\left(\mathrm{~d}, J_{A-B}=12.1 \mathrm{~Hz}, 1 \mathrm{H}\right), 4.25\left(\mathrm{~d}, J_{A-B}=\right.$ $12.1 \mathrm{~Hz}, 1 \mathrm{H}), 2.77(\mathrm{dd}, J=13.3,7.4 \mathrm{~Hz}, 1 \mathrm{H}), 2.51$ (dd, $J=13.6,5.6 \mathrm{~Hz}, 1 \mathrm{H}), 1.26$ (s, $12 \mathrm{H}), 1.22(\mathrm{~s}, 6 \mathrm{H}), 1.20(\mathrm{~s}, 6 \mathrm{H}) .{ }^{13} \mathrm{C} \mathrm{NMR}\left(151 \mathrm{MHz}, \mathrm{CDCl}_{3}\right) \delta 141.0,138.7,133.3$, $128.8,128.7,128.5,128.0,127.7,83.9,83.6,80.3,70.5,48.9,25.19,25.17,25.15$. HRMS $\left(\mathrm{ESI}^{+}\right): \mathrm{m} / \mathrm{z}$ for $\mathrm{C}_{29} \mathrm{H}_{43} \mathrm{~B}_{2} \mathrm{ClNO}_{5}\left[\mathrm{M}+\mathrm{NH}_{4}\right]^{+}$calcd. 542.3016, found 542.3019.

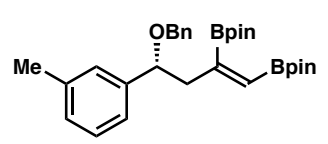

$(R, E)-2,2 '-(4-(B e n z y l o x y)-4-(m-t o l y l) b u t-1-e n e-1,2-d i y l) b i s(4,4,5$, 5-tetramethyl-1,3,2-dioxaborolane) (4g) Prepared according to the general procedure. The crude mixture was purified by column chromatography with hexane and ethyl acetate to give the title compound as colorless oil in $85 \%$ yield $(43 \mathrm{mg}, E / Z=7 / 1) .{ }^{1} \mathrm{H}$ NMR $\left(600 \mathrm{MHz}, \mathrm{CDCl}_{3}\right) \delta 7.20-7.32(\mathrm{~m}, 6 \mathrm{H}), 7.15$ (s, 1H), 7.12 (d, $J=7.4 \mathrm{~Hz}, 1 \mathrm{H}), 7.07$ (d, $J=7.3 \mathrm{~Hz}, 1 \mathrm{H}), 5.91(\mathrm{~s}, 1 \mathrm{H}), 4.41-4.45$ (m, $2 \mathrm{H}), 4.27\left(\mathrm{~d}, J_{A-B}=12.1 \mathrm{~Hz}, 1 \mathrm{H}\right), 2.78(\mathrm{dd}, J=13.6,8.5 \mathrm{~Hz}, 1 \mathrm{H}), 2.52(\mathrm{dd}, J=13.8,3.9$ $\mathrm{Hz}, 1 \mathrm{H}), 2.34$ (s, 3H), 1.27 (s, 12H), 1.23 (s, 6H), 1.21 (s, 6H). ${ }^{13} \mathrm{C}$ NMR (151 MHz, $\left.\mathrm{CDCl}_{3}\right) \delta 142.7,139.1,138.2,128.50,128.47,128.42,128.0,127.8,127.4,124.3,83.8$, 83.6, 81.2, 70.4, 49.1, 25.21, 25.16, 25.15, 21.9. HRMS (ESI $\left.{ }^{+}\right): \mathrm{m} / \mathrm{z}$ for $\mathrm{C}_{30} \mathrm{H}_{46} \mathrm{~B}_{2} \mathrm{NO}_{5}$ $\left[\mathrm{M}+\mathrm{NH}_{4}\right]^{+}$calcd. 522.3562, found 522.3539.

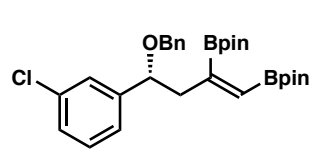

(R,E)-2,2'-(4-(Benzyloxy)-4-(3-chlorophenyl)but-1-ene-1,2-diyl)bi s(4,4,5,5-tetramethyl-1,3,2-dioxaborolane) (4h) Prepared according to the general procedure. The crude mixture was purified by column chromatography with hexane and ethyl acetate to give the title compound as colorless oil 
in $82 \%$ yield $(43 \mathrm{mg}, E / Z=11 / 1) .{ }^{1} \mathrm{H} \mathrm{NMR}\left(600 \mathrm{MHz}, \mathrm{CDCl}_{3}\right) \delta 7.19-7.33(\mathrm{~m}, 9 \mathrm{H})$, $5.87(\mathrm{~s}, 1 \mathrm{H}), 4.42-4.44(\mathrm{~m}, 2 \mathrm{H}), 4.27\left(\mathrm{~d}, J_{A-B}=12.0 \mathrm{~Hz}, 1 \mathrm{H}\right), 2.75(\mathrm{dd}, J=13.6,7.9 \mathrm{~Hz}$, $1 \mathrm{H}), 2.51(\mathrm{dd}, J=13.6,5.1 \mathrm{~Hz}, 1 \mathrm{H}), 1.27(\mathrm{~s}, 12 \mathrm{H}), 1.23(\mathrm{~s}, 6 \mathrm{H}), 1.20(\mathrm{~s}, 6 \mathrm{H}) .{ }^{13} \mathrm{C} \mathrm{NMR}$ $\left(151 \mathrm{MHz}, \mathrm{CDCl}_{3}\right) \delta 144.9,138.7,134.4,129.9,128.5,128.0,127.9,127.7,127.3,125.5$, 83.9, 83.7, 80.5, 70.7, 48.9, 25.19, 25.16. HRMS $\left(\mathrm{ESI}^{+}\right): \mathrm{m} / \mathrm{z}$ for $\mathrm{C}_{29} \mathrm{H}_{43} \mathrm{~B}_{2} \mathrm{ClNO}_{5}$ $\left[\mathrm{M}+\mathrm{NH}_{4}\right]^{+}$calcd. 542.3016, found 542.3018.

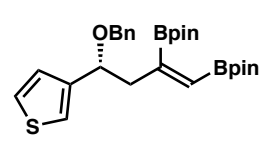

$(R, E)-2,2^{\prime}-(4-(B e n z y l o x y)-4-(t h i o p h e n-3-y l) b u t-1-e n e-1,2-d i y l) b i s(4,4$ ,5,5-tetramethyl-1,3,2-dioxaborolane) (4i) Prepared according to the general procedure. The crude mixture was purified by column chromatography with hexane and ethyl acetate to give the title compound as colorless oil in $89 \%$ yield (44 mg, $E / Z=13 / 1) .{ }^{1} \mathrm{H}$ NMR (600 MHz, $\left.\mathrm{CDCl}_{3}\right) \delta 7.23-7.30(\mathrm{~m}, 6 \mathrm{H})$, $7.14(\mathrm{~s}, 1 \mathrm{H}), 7.09(\mathrm{~d}, J=4.8 \mathrm{~Hz}, 1 \mathrm{H}), 5.86(\mathrm{~s}, 1 \mathrm{H}), 4.58(\mathrm{dd}, J=6.6,6.5 \mathrm{~Hz}, 1 \mathrm{H}), 4.46$ (d, $\left.J_{A-B}=12.1 \mathrm{~Hz}, 1 \mathrm{H}\right), 4.31\left(\mathrm{~d}, J_{A-B}=12.0 \mathrm{~Hz}, 1 \mathrm{H}\right), 2.84(\mathrm{dd}, J=13.6,7.4 \mathrm{~Hz}, 1 \mathrm{H}), 2.59$ $(\mathrm{dd}, J=13.7,5.8 \mathrm{~Hz}, 1 \mathrm{H}), 1.26(\mathrm{~s}, 12 \mathrm{H}), 1.23(\mathrm{~s}, 6 \mathrm{H}), 1.21(\mathrm{~s}, 6 \mathrm{H}) .{ }^{13} \mathrm{C}$ NMR $(151 \mathrm{MHz}$, $\left.\mathrm{CDCl}_{3}\right) \delta 143.8,139.0,128.5,128.0,127.6,126.6,126.1,122.5,83.9,83.6,76.7,70.4$, 48.1, 25.20, 25.16, 25.15. HRMS $\left(\mathrm{ESI}^{+}\right): \mathrm{m} / \mathrm{z}$ for $\mathrm{C}_{27} \mathrm{H}_{42} \mathrm{~B}_{2} \mathrm{SNO}_{5}\left[\mathrm{M}+\mathrm{NH}_{4}\right]^{+}$calcd. 514.2970, found 514.2975.

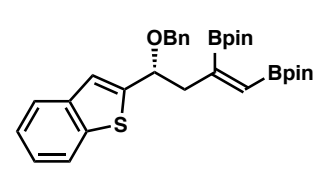

$(R, E)-2,2$ '-(4-(Benzo[b]thiophen-2-yl)-4-(benzyloxy)but-1-ene-1,2diyl)bis(4,4,5,5-tetramethyl-1,3,2-dioxaborolane) (4j) Prepared according to the general procedure. The crude mixture was purified by column chromatography with hexane and ethyl acetate to give the title compound as colorless oil in $81 \%$ yield $(44 \mathrm{mg}, E / Z=9 / 1) .{ }^{1} \mathrm{H} \mathrm{NMR}\left(600 \mathrm{MHz}, \mathrm{CDCl}_{3}\right) \delta 7.81(\mathrm{~d}, J=$ $7.7 \mathrm{~Hz}, 1 \mathrm{H}), 7.71(\mathrm{~d}, J=7.6 \mathrm{~Hz}, 1 \mathrm{H}), 7.17-7.35$ (m, 7H), $7.17(\mathrm{~s}, 1 \mathrm{H}), 5.94(\mathrm{~s}, 1 \mathrm{H})$, $4.80(\mathrm{dd}, J=6.6,6.5 \mathrm{~Hz}, 1 \mathrm{H}), 4.57\left(\mathrm{~d}, J_{A-B}=12.0 \mathrm{~Hz}, 1 \mathrm{H}\right), 4.37\left(\mathrm{~d}, J_{A-B}=11.9 \mathrm{~Hz}, 1 \mathrm{H}\right)$, 2.95 (dd, $J=13.6,7.1 \mathrm{~Hz}, 1 \mathrm{H}), 2.73$ (dd, $J=13.7,6.0 \mathrm{~Hz}, 1 \mathrm{H}), 1.25$ (s, 12H), 1.20 (s, $6 \mathrm{H}), 1.19(\mathrm{~s}, 6 \mathrm{H}) .{ }^{13} \mathrm{C} \mathrm{NMR}\left(151 \mathrm{MHz}, \mathrm{CDCl}_{3}\right) \delta 147.5,140.1,139.7,138.5,134.1,128.6$, 128.2, 127.7, 124.3 (2 C signals), 123.7, 122.9, 122.5, 84.0, 83.7, 77.0, 70.5, 48.7, 25.2, 25.1. HRMS $\left(\mathrm{ESI}^{+}\right): \mathrm{m} / \mathrm{z}$ for $\mathrm{C}_{31} \mathrm{H}_{44} \mathrm{~B}_{2} \mathrm{SNO}_{5}\left[\mathrm{M}+\mathrm{NH}_{4}\right]^{+}$calcd. 564.3126, found 564.3136. 


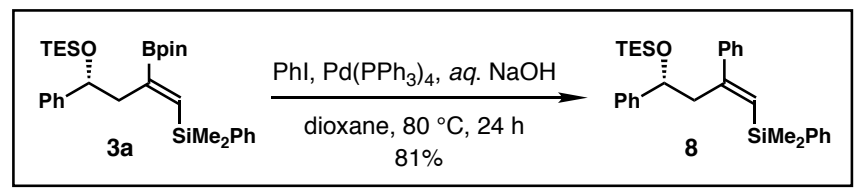

(R,E)-((4-(Dimethyl(phenyl)silyl)-1,3-diphenylbut-3-en-1-yl)oxy)triethylsilane (8): In an Ar-filled glove box, $\mathrm{Pd}\left(\mathrm{PPh}_{3}\right)_{4}(0.01 \mathrm{mmol}, 5 \mathrm{~mol} \%)$, vinylboronate 3a $(0.2 \mathrm{mmol}$, 1.0 equiv), iodobenzene ( $0.24 \mathrm{mmol}, 1.2$ equiv), and 1,4-dioxane (1 mL) were sequentially added to a 1-dram vial equipped with a Teflon-coated magnetic stir bar. The vial was sealed with a rubber septum and removed from the glove box. Aqueous $\mathrm{NaOH}$ ( $3 \mathrm{M}, 0.2 \mathrm{~mL}$ ) was added to the mixture, and the via was sealed with PTFE/silicone-lined septum cap. After stirring at $80{ }^{\circ} \mathrm{C}$ in an oil bath for $24 \mathrm{~h}$, the reaction mixture was cooled to ambient temperature and filtered through a pad of silica gel. The solution was concentrated under reduced pressure. Purification of the crude product was performed by flash column chromatography (hexane/ethyl acetate) to give compound $\mathbf{8}$ as colorless oil in $81 \%$ yield $(77 \mathrm{mg}) .{ }^{1} \mathrm{H} \mathrm{NMR}\left(400 \mathrm{MHz}, \mathrm{CDCl}_{3}\right) \delta 7.53-7.54(\mathrm{~m}, 2 \mathrm{H}), 7.46(\mathrm{~d}, J=7.6$ $\mathrm{Hz}, 2 \mathrm{H}), 7.17-7.38(\mathrm{~m}, 9 \mathrm{H}), 7.14(\mathrm{~d}, J=7.6 \mathrm{~Hz}, 2 \mathrm{H}), 5.96(\mathrm{~s}, 1 \mathrm{H}), 4.60(\mathrm{dd}, J=8.0,4.9$ $\mathrm{Hz}, 1 \mathrm{H}), 3.05$ (dd, $J=14.2,8.4 \mathrm{~Hz}, 1 \mathrm{H}), 2.83$ (dd, $J=14.1,4.9 \mathrm{~Hz}, 1 \mathrm{H}), 0.72$ (t, $J=7.9$ $\mathrm{Hz}, 9 \mathrm{H}), 0.394$ (s, 3H), 0.386 (s, 3H), 0.27 (q, $J=7.8 \mathrm{~Hz}, 6 \mathrm{H}) .{ }^{13} \mathrm{C}$ NMR (151 MHz, $\left.\mathrm{CDCl}_{3}\right) \delta 155.3,145.9,143.8,140.1,134.2,129.6,129.1,128.5,128.3,128.1,127.8$, 127.3, 126.9, 126.1, 73.6, 46.3, 7.1, 4.9, -0.2, -0.3. HRMS $\left(\mathrm{ESI}^{+}\right): \mathrm{m} / \mathrm{z}$ for $\mathrm{C}_{30} \mathrm{H}_{40} \mathrm{OSi}_{2} \mathrm{Na}$ $[\mathrm{M}+\mathrm{Na}]^{+}$calcd. 495.2515, found 495.2537.

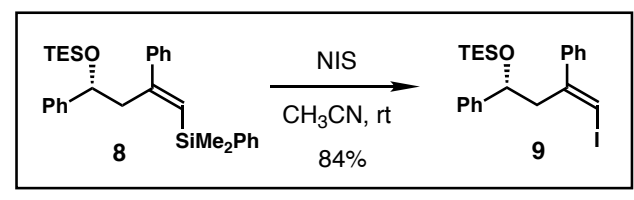

$(\boldsymbol{R}, E)$-Triethyl((4-iodo-1,3-diphenylbut-3-en-1-yl)oxy)silane (9): To a solution of vinylsilane $8(0.1 \mathrm{mmol}, 1.0$ equiv) in acetonitrile $(0.5 \mathrm{~mL})$ was added $N$-iodosuccinimide (NIS, $0.2 \mathrm{mmol}, 2.0$ equiv). The reaction mixture was stirred at ambient temperature and the progress was monitored by TLC analysis. After complete consumption of the starting vinylsilane 8, saturated $\mathrm{Na}_{2} \mathrm{~S}_{2} \mathrm{O}_{3}(2 \mathrm{~mL})$ was added, and the resulting mixture was stirred vigorously until it became colorless. The reaction mixture was diluted with $\mathrm{Et}_{2} \mathrm{O}(5 \mathrm{~mL})$. The organic layer was separated and the aqueous layer was extracted with $\operatorname{Et}_{2} \mathrm{O}(3 \times 1$ $\mathrm{mL}$ ). The combined organic layers were dried over anhydrous sodium sulfate, filtered, and concentrated under reduced pressure. Purification of the crude reaction product was performed by flash column chromatography (hexane/ethyl acetate) to give compound 9 
as colorless oil in $84 \%$ yield (39 mg). ${ }^{1} \mathrm{H}$ NMR (400 $\left.\mathrm{MHz}, \mathrm{CDCl}_{3}\right) \delta 7.19-7.35(\mathrm{~m}$, $10 \mathrm{H}), 6.54$ (s, 1H), 4.88 (dd, $J=8.0,5.5 \mathrm{~Hz}, 1 \mathrm{H}), 3.15$ (dd, $J=13.7,8.0 \mathrm{~Hz}, 1 \mathrm{H}), 2.98$ $(\mathrm{dd}, J=13.7,5.5 \mathrm{~Hz}, 1 \mathrm{H}), 0.77(\mathrm{t}, J=7.9 \mathrm{~Hz}, 9 \mathrm{H}), 0.35-0.41(\mathrm{~m}, 6 \mathrm{H}) .{ }^{13} \mathrm{C}$ NMR $(151$ $\left.\mathrm{MHz}_{\mathrm{CDCl}}\right) \delta 149.0,145.0,141.5,128.6,128.3,128.0,127.5,127.1,126.3,81.6,73.5$, 48.4, 7.1, 4.9. HRMS $\left(\mathrm{ESI}^{+}\right): \mathrm{m} / \mathrm{z}$ for $\mathrm{C}_{22} \mathrm{H}_{29} \mathrm{IOSiNa}[\mathrm{M}+\mathrm{Na}]^{+}$calcd. 487.0930, found 487.0924 .

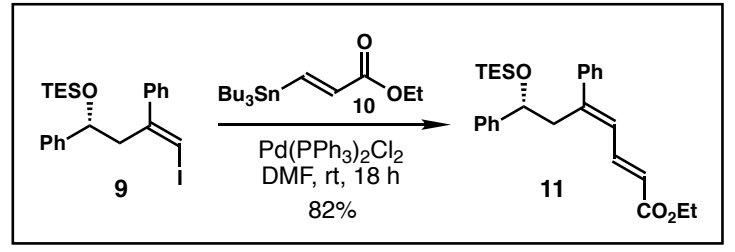

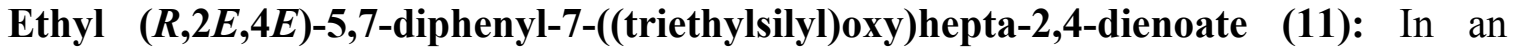
Ar-filled glove box, bis(triphenylphosphine)palladium(II) dichloride (0.0025 mmol, 5 mol \%), vinyl iodide 9 ( $0.05 \mathrm{mmol}, 1.0$ equiv) and a Teflon-coated magnetic stirring bar were sequentially added into a reaction flask. The flask was capped with a rubber septum and removed from glove box. $E$-vinylstannane 10 (0.075 mmol, 1.5 equiv) in DMF (0.2 $\mathrm{mL}$ ) was added to the reaction mixture. The reaction was protected from light and stirred at ambient temperature for $18 \mathrm{~h}$. After complete consumption of vinyl iodide 9, the reaction mixture was diluted with $\mathrm{Et}_{2} \mathrm{O}(5 \mathrm{~mL})$ and washed with water $(3 \mathrm{x} 1 \mathrm{~mL})$. The organic layer was dried over anhydrous $\mathrm{Na}_{2} \mathrm{SO}_{4}$, filtered, and concentrated under reduced pressure. Purification of the crude product was performed by flash chromatography with hexane and ethyl acetate to give compound 11 as colorless oil in $82 \%$ yield $(18 \mathrm{mg}) .{ }^{1} \mathrm{H}$ NMR (600 MHz, $\left.\mathrm{CDCl}_{3}\right) \delta 7.63(\mathrm{dd}, J=15.0,11.7 \mathrm{~Hz}, 1 \mathrm{H}), 7.48(\mathrm{~d}, J=7.4 \mathrm{~Hz}, 2 \mathrm{H})$, $7.38(\mathrm{t}, J=7.4 \mathrm{~Hz}, 2 \mathrm{H}), 7.33(\mathrm{t}, J=7.2 \mathrm{~Hz}, 1 \mathrm{H}), 7.26-7.29(\mathrm{~m}, 4 \mathrm{H}), 7.20-7.22(\mathrm{~m}$, $1 \mathrm{H}), 6.52(\mathrm{~d}, J=11.6 \mathrm{~Hz}, 1 \mathrm{H}), 5.89(\mathrm{~d}, J=15.1 \mathrm{~Hz}, 1 \mathrm{H}), 4.65(\mathrm{dd}, J=7.9,4.9 \mathrm{~Hz}, 1 \mathrm{H})$, $4.23(\mathrm{q}, J=7.0,6.5 \mathrm{~Hz}, 2 \mathrm{H}), 3.19(\mathrm{dd}, J=13.9,8.1 \mathrm{~Hz}, 1 \mathrm{H}), 3.00(\mathrm{dd}, J=13.9,4.8 \mathrm{~Hz}$, $1 \mathrm{H}), 1.33(\mathrm{t}, J=7.1 \mathrm{~Hz}, 3 \mathrm{H}), 0.74(\mathrm{t}, J=7.9 \mathrm{~Hz}, 9 \mathrm{H}), 0.30-0.40(\mathrm{~m}, 6 \mathrm{H}) .{ }^{13} \mathrm{C} \mathrm{NMR}$ $\left(151 \mathrm{MHz} \mathrm{CDCl}_{3}\right) \delta 167.6,146.9,145.1,141.6,141.4,128.8,128.6,128.4,127.7,127.6$, 127.0, 126.0, 121.8, 74.5, 60.6, 42.4, 14.7, 7.0, 4.9. HRMS (ESI $\left.{ }^{+}\right): \mathrm{m} / \mathrm{z}$ for $\mathrm{C}_{27} \mathrm{H}_{36} \mathrm{O}_{3} \mathrm{SiNa}$ $[\mathrm{M}+\mathrm{Na}]^{+}$calcd. 459.2331, found 459.2338. 


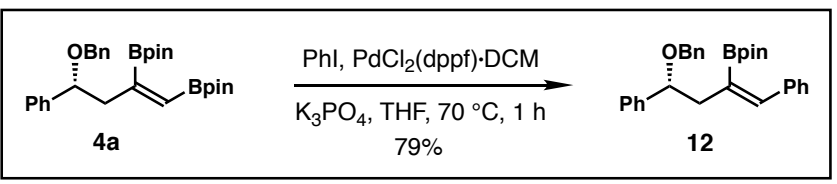

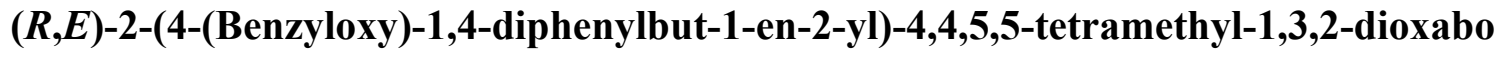
rolane (12): In an Ar-filled glove box, $\mathrm{PdCl}_{2}(\mathrm{dppf}) \cdot \mathrm{CH}_{2} \mathrm{Cl}_{2}(0.005 \mathrm{mmol}, 5 \mathrm{~mol} \%)$, vinylboronate $4 \mathbf{a}\left(0.1 \mathrm{mmol}, 1.0\right.$ equiv), iodobenzene ( $0.1 \mathrm{mmol}, 1.0$ equiv), $\mathrm{K}_{3} \mathrm{PO}_{4}(0.12$ mmol, 1.2 equiv), THF $(0.5 \mathrm{~mL})$ and a Teflon-coated magnetic stirring bar were sequentially added into a 1-dram vial. Water $(0.1 \mathrm{~mL})$ was added to the mixture under an argon atmosphere. The vial was sealed with a cap containing a PTFE-lined silicone septum and stirred at $70{ }^{\circ} \mathrm{C}$ in an oil bath for $1 \mathrm{~h}$. After complete consumption of $\mathbf{4 a}$, the resulting mixture was filtered through a short pad of Celite. Brine $(1 \mathrm{~mL})$ was added and the mixture was extracted with $\mathrm{Et}_{2} \mathrm{O}(3 \times 1 \mathrm{~mL})$. The combined organic layers were dried over anhydrous $\mathrm{Na}_{2} \mathrm{SO}_{4}$, filtered, and concentrated under reduced pressure. Purification of crude reaction product was performed by flash chromatography with hexane and ethyl acetate to provide compound $\mathbf{1 2}$ as colorless oil in 79\% yield (35 mg). ${ }^{1} \mathrm{H}$ NMR (600 $\left.\mathrm{MHz}, \mathrm{CDCl}_{3}\right) \delta 7.36$ (app. d, $\left.J=4.2 \mathrm{~Hz}, 4 \mathrm{H}\right), 7.19-7.33(\mathrm{~m}, 11 \mathrm{H}), 6.75(\mathrm{~s}, 1 \mathrm{H}), 4.49-$ $4.51(\mathrm{~m}, 2 \mathrm{H}), 4.29\left(\mathrm{~d}, J_{A-B}=12.1 \mathrm{~Hz}, 1 \mathrm{H}\right), 2.84(\mathrm{dd}, J=13.1,7.6 \mathrm{~Hz}, 1 \mathrm{H}), 2.61$ (dd, $J=$ 13.2, $5.5 \mathrm{~Hz}, 1 \mathrm{H}), 1.19$ (s, 6H), $1.18(\mathrm{~s}, 6 \mathrm{H}) .{ }^{13} \mathrm{C} \mathrm{NMR}\left(151 \mathrm{MHz}, \mathrm{CDCl}_{3}\right) \delta 143.9,142.5$, $139.0,138.9,128.61,128.58,128.56,128.2$, 128.1, 127.74, 127.66, 127.4, 127.3, 83.7, 81.5, 70.7, 47.4, 25.1, 25.0. HRMS $\left(\mathrm{ESI}^{+}\right): \mathrm{m} / \mathrm{z}$ for $\mathrm{C}_{29} \mathrm{H}_{33} \mathrm{BO}_{3} \mathrm{Na}[\mathrm{M}+\mathrm{Na}]^{+}$calcd. 463.2420, found 463.2406 .

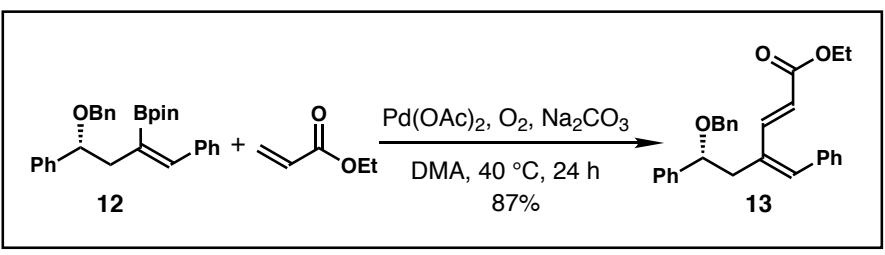

Ethyl (R,E)-4-((Z)-benzylidene)-6-(benzyloxy)-6-phenylhex-2-enoate (13): To a solution of ethyl acrylate ( $0.15 \mathrm{mmol}, 3.0$ equiv) in $N, N$-dimethylacetamide (DMA, 0.25 $\mathrm{mL}$ ) was added vinylboronate 12 (0.05 mmol, 1.0 equiv), $\mathrm{Na}_{2} \mathrm{CO}_{3}$ (0.2 mmol, 2.0 equiv) and $\mathrm{Pd}(\mathrm{OAc})_{2}(0.005 \mathrm{mmol}, 10 \mathrm{~mol} \%)$. The reaction flask was fitted with an oxygen balloon. After stirring at $40{ }^{\circ} \mathrm{C}$ in an oil bath for $24 \mathrm{~h}$, the reaction mixture was diluted with $\mathrm{Et}_{2} \mathrm{O}(5 \mathrm{~mL})$, and washed with water $(3 \times 1 \mathrm{~mL})$. The organic layer was dried over anhydrous $\mathrm{Na}_{2} \mathrm{SO}_{4}$, filtered and concentrated under reduced pressure. Purification of the crude reaction product was performed by flash chromatography with hexane and ethyl 
acetate to provide compound $\mathbf{1 3}$ as colorless oil in $87 \%$ yield $\left(18 \mathrm{mg}\right.$ ). ${ }^{1} \mathrm{H}$ NMR (400 $\left.\mathrm{MHz}, \mathrm{CDCl}_{3}\right) \delta 7.72(\mathrm{~d}, J=15.8 \mathrm{~Hz}, 1 \mathrm{H}), 7.26-7.40(\mathrm{~m}, 13 \mathrm{H}), 7.13(\mathrm{~d}, J=7.3 \mathrm{~Hz}, 2 \mathrm{H})$, $6.60(\mathrm{~s}, 1 \mathrm{H}), 6.07(\mathrm{~d}, J=16.0 \mathrm{~Hz}, 1 \mathrm{H}), 4.53(\mathrm{dd}, J=7.4,6.0 \mathrm{~Hz}, 1 \mathrm{H}), 4.50\left(\mathrm{~d}, J_{A-B}=11.8\right.$ $\mathrm{Hz}, 1 \mathrm{H}), 4.28\left(\mathrm{~d}, J_{A-B}=11.9 \mathrm{~Hz}, 1 \mathrm{H}\right), 4.20(\mathrm{q}, J=7.1 \mathrm{~Hz}, 2 \mathrm{H}), 2.97$ (dd, $J=14.0,7.5 \mathrm{~Hz}$, $1 \mathrm{H}), 2.67(\mathrm{dd}, J=14.2,5.7 \mathrm{~Hz}, 1 \mathrm{H}), 1.30(\mathrm{t}, J=7.1 \mathrm{~Hz}, 3 \mathrm{H}) .{ }^{13} \mathrm{C}$ NMR $(151 \mathrm{MHz}$, $\left.\mathrm{CDCl}_{3}\right) \delta 167.7,142.3,142.0,139.8,138.5,136.6,133.1,129.8,128.8,128.7,128.6$, 128.2, 128.0 (2 C signals), 127.9, 127.1, 119.7, 80.2, 70.9, 60.7, 43.5, 14.7. HRMS (ESI ${ }^{+}$): $\mathrm{m} / \mathrm{z}$ for $\mathrm{C}_{28} \mathrm{H}_{28} \mathrm{O}_{3} \mathrm{Na}[\mathrm{M}+\mathrm{Na}]^{+}$calcd. 435.1936, found 435.1953 .

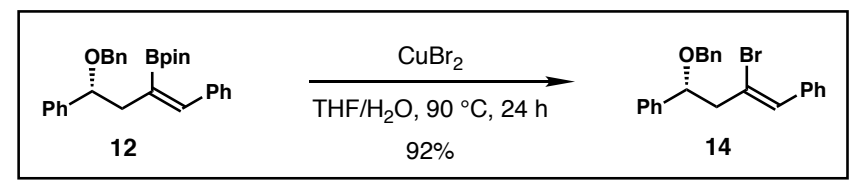

$(R, Z)$-(4-(Benzyloxy)-2-bromobut-1-ene-1,4-diyl)dibenzene (14): To a reaction vial containing a Teflon-coated magnetic stirring bar was added vinylboronate $12(0.05 \mathrm{mmol}$, 1.0 equiv). THF $(0.3 \mathrm{~mL})$ and $\mathrm{H}_{2} \mathrm{O}(0.3 \mathrm{~mL})$ were added to the vial followed by addition of $\mathrm{CuBr}_{2}\left(0.3 \mathrm{mmol}, 3.0\right.$ equiv). The reaction mixture was stirred at $90{ }^{\circ} \mathrm{C}$ in an oil bath for $24 \mathrm{~h}$. After cooling to ambient temperature, $\mathrm{H}_{2} \mathrm{O}(0.5 \mathrm{~mL})$ was added to the vial, and the reaction mixture was extracted with $\mathrm{Et}_{2} \mathrm{O}(3 \mathrm{x} 1 \mathrm{~mL})$. The combined organic layers were dried over anhydrous $\mathrm{Na}_{2} \mathrm{SO}_{4}$, filtered, and concentrated under reduced pressure. Purification of the crude product was performed by flash column chromatography (hexane/ethyl acetate) to give product 14 as colorless oil in $92 \%$ yield $(18 \mathrm{mg}) .{ }^{1} \mathrm{H} \mathrm{NMR}$ $\left(600 \mathrm{MHz}, \mathrm{CDCl}_{3}\right) \delta 7.47(\mathrm{~d}, J=7.5 \mathrm{~Hz}, 2 \mathrm{H}), 7.38-7.42(\mathrm{~m}, 4 \mathrm{H}), 7.26-7.36(\mathrm{~m}, 9 \mathrm{H})$, $6.68(\mathrm{~s}, 1 \mathrm{H}), 4.81(\mathrm{dd}, J=8.1,5.1 \mathrm{~Hz}, 1 \mathrm{H}), 4.53\left(\mathrm{~d}, J_{A-B}=11.8 \mathrm{~Hz}, 1 \mathrm{H}\right), 4.35\left(\mathrm{~d}, J_{A-B}=\right.$ $11.7 \mathrm{~Hz}, 1 \mathrm{H}), 3.15(\mathrm{dd}, J=14.2,8.3 \mathrm{~Hz}, 1 \mathrm{H}), 2.84(\mathrm{dd}, J=14.3,4.9 \mathrm{~Hz}, 1 \mathrm{H}) .{ }^{13} \mathrm{C} \mathrm{NMR}$ $\left(151 \mathrm{MHz}, \mathrm{CDCl}_{3}\right) \delta 141.4,138.5,136.3,130.6,129.1,128.9,128.7,128.3,128.2,128.1$, 128.0, 127.9, 127.0, 123.3, 79.4, 71.2, 52.4. HRMS $\left(\mathrm{ESI}^{+}\right): \mathrm{m} / \mathrm{z}$ for $\mathrm{C}_{23} \mathrm{H}_{25} \mathrm{BrNO}$ $\left[\mathrm{M}+\mathrm{NH}_{4}\right]^{+}$calcd. 410.1120 , found 410.1112 .

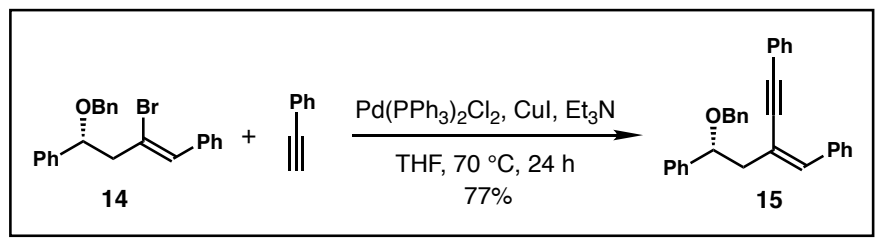

$(R, Z)$-(3-Benzylidene-5-(benzyloxy)pent-1-yne-1,5-diyl)dibenzene (15): In an Ar-filled glove box, bis(triphenylphosphine)palladium(II) dichloride (0.005 mmol, $10 \mathrm{~mol} \%$ ), $\mathrm{CuI}$ 
(0.01 mmol, $20 \mathrm{~mol} \%)$ and a Teflon-coated magnetic stirring bar were sequentially added into a reaction vial. The vial was capped with a rubber septum and removed from glove box. A solution of vinyl bromine 14 (0.05 mmol, 1.0 equiv) in $\mathrm{Et}_{3} \mathrm{~N}(0.1 \mathrm{~mL})$ was added and the mixture was stirred at ambient temperature for $30 \mathrm{~min}$. Then a solution of phenylacetylene $(0.10 \mathrm{mmol}, 2$ equiv) in THF $(0.1 \mathrm{~mL})$ was added and the via was sealed with PTFE/silicone-lined septum cap. After stirring at $70{ }^{\circ} \mathrm{C}$ in an oil bath for $24 \mathrm{~h}$, the reaction mixture was cooled to ambient temperature and filtered through a pad of silica gel. The solution was concentrated under reduced pressure. Purification of the crude reaction product was performed by flash column chromatography (hexane/ethyl acetate) to give product 15 as colorless oil in $77 \%$ yield $(16 \mathrm{mg}){ }^{1} \mathrm{H} \mathrm{NMR}\left(600 \mathrm{MHz}, \mathrm{CDCl}_{3}\right) \delta$ $7.82(\mathrm{~d}, J=7.7 \mathrm{~Hz}, 2 \mathrm{H}), 7.21-7.43(\mathrm{~m}, 18 \mathrm{H}), 6.54(\mathrm{~s}, 1 \mathrm{H}), 4.82(\mathrm{dd}, J=7.6,5.9 \mathrm{~Hz}$, $1 \mathrm{H}), 4.54\left(\mathrm{~d}, J_{A-B}=11.9 \mathrm{~Hz}, 1 \mathrm{H}\right), 4.36\left(\mathrm{~d}, J_{A-B}=11.9 \mathrm{~Hz}, 1 \mathrm{H}\right), 2.95(\mathrm{dd}, J=13.6,8.1 \mathrm{~Hz}$, $1 \mathrm{H}), 2.70(\mathrm{dd}, J=13.6,5.4 \mathrm{~Hz}, 1 \mathrm{H}) .{ }^{13} \mathrm{C} \mathrm{NMR}\left(151 \mathrm{MHz}, \mathrm{CDCl}_{3}\right) \delta 142.0,138.6,137.0$, 136.9, 131.8, 128.81, 128.79, 128.67, 128.63, 128.61, 128.5, 128.14, 128.12, 128.0, 127.8, 127.2, 123.7, 118.6, 96.2, 89.7, 80.2, 71.1, 48.5. HRMS $\left(\mathrm{ESI}^{+}\right): \mathrm{m} / \mathrm{z}$ for $\mathrm{C}_{31} \mathrm{H}_{26} \mathrm{ONa}$ $[\mathrm{M}+\mathrm{Na}]^{+}$calcd. 437.1881, found 437.1880.

\section{References:}

1. Vyas, D.J.; Hazra, C. K.; Oestreich, M. Org. Lett. 2011, 13, 4462. 


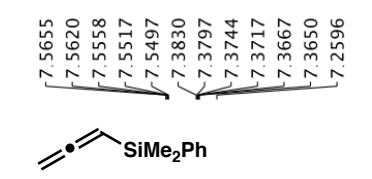

$\left(\mathrm{CDCl}_{3}, 600 \mathrm{MHz}\right)$
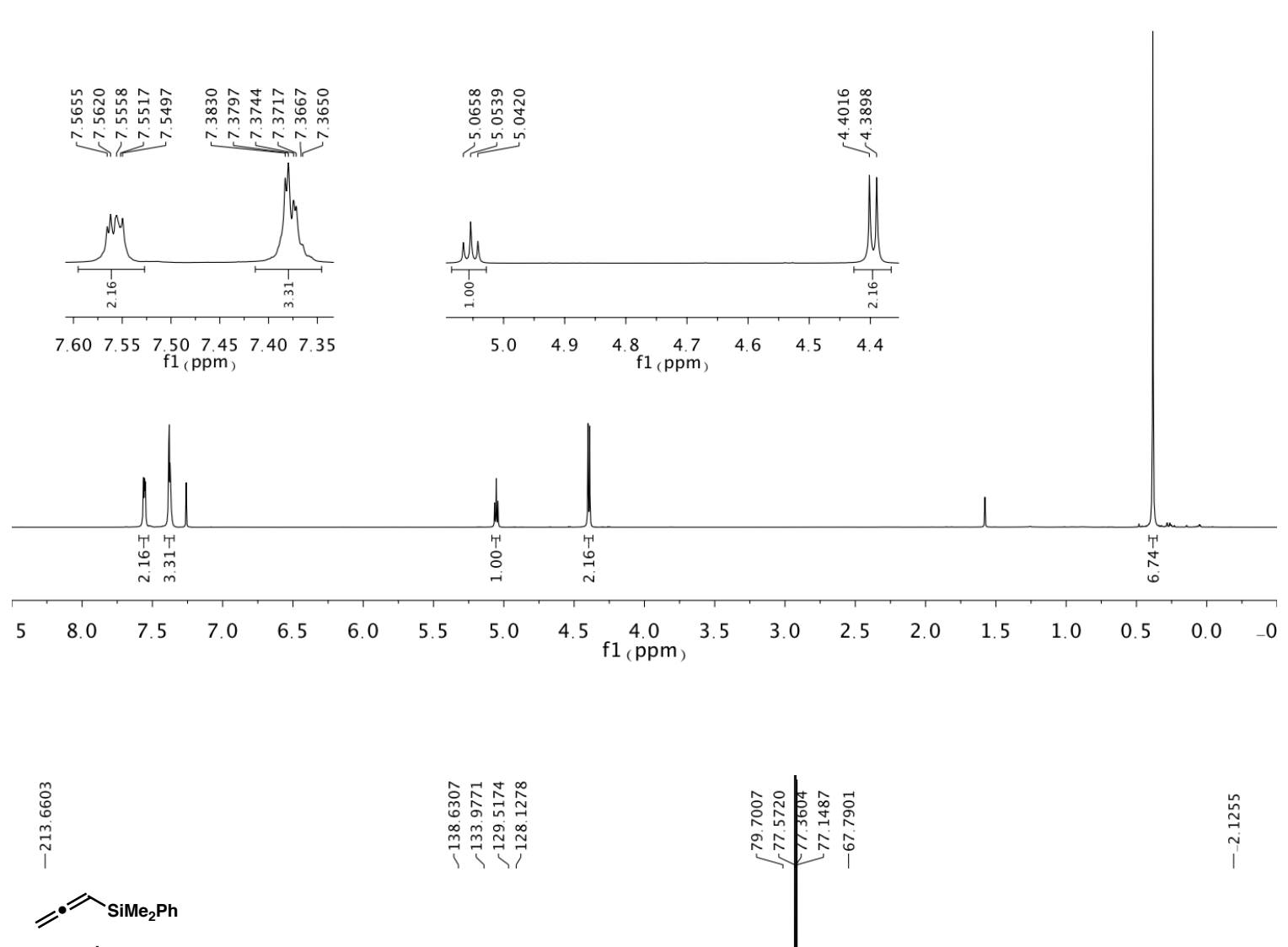

$\left(\mathrm{CDCl}_{3}, \begin{array}{c}1 \\ 151 \mathrm{MHz})\end{array}\right.$
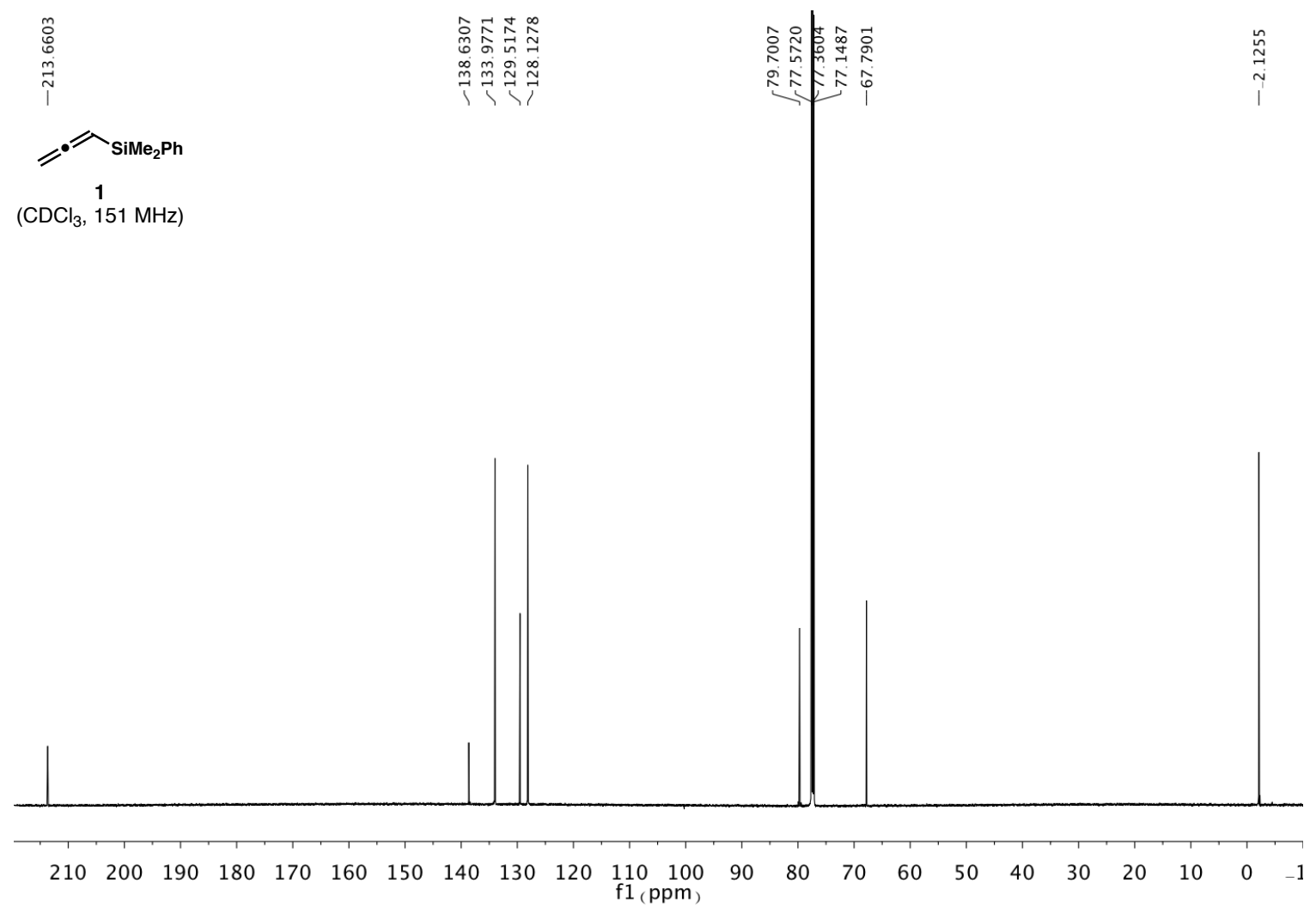

SI-19 


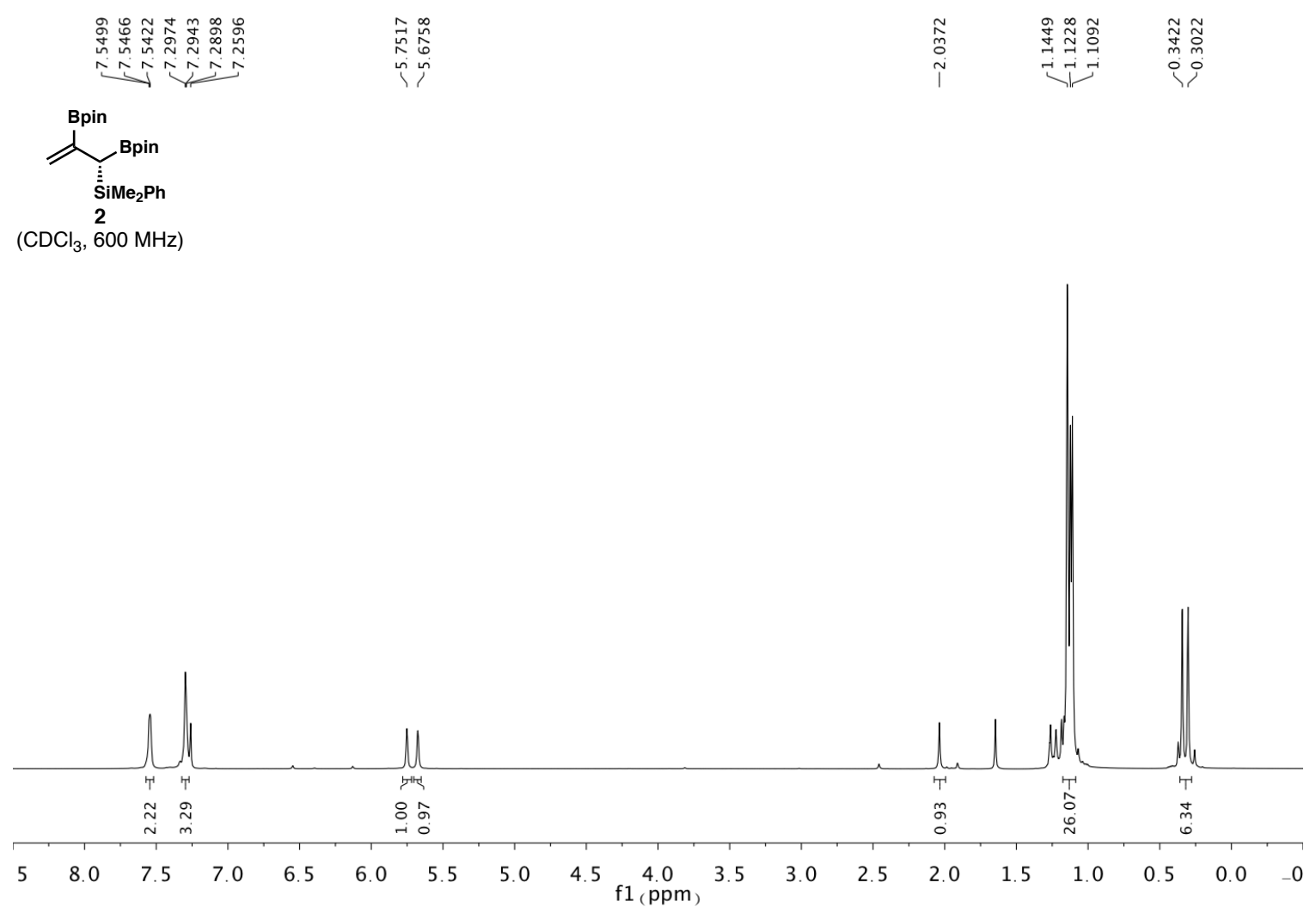

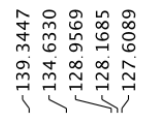

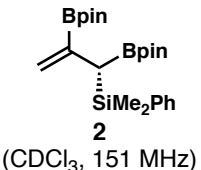

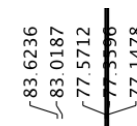

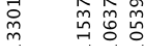

弪 $\stackrel{\sim}{\sim}$

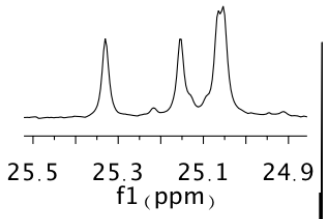

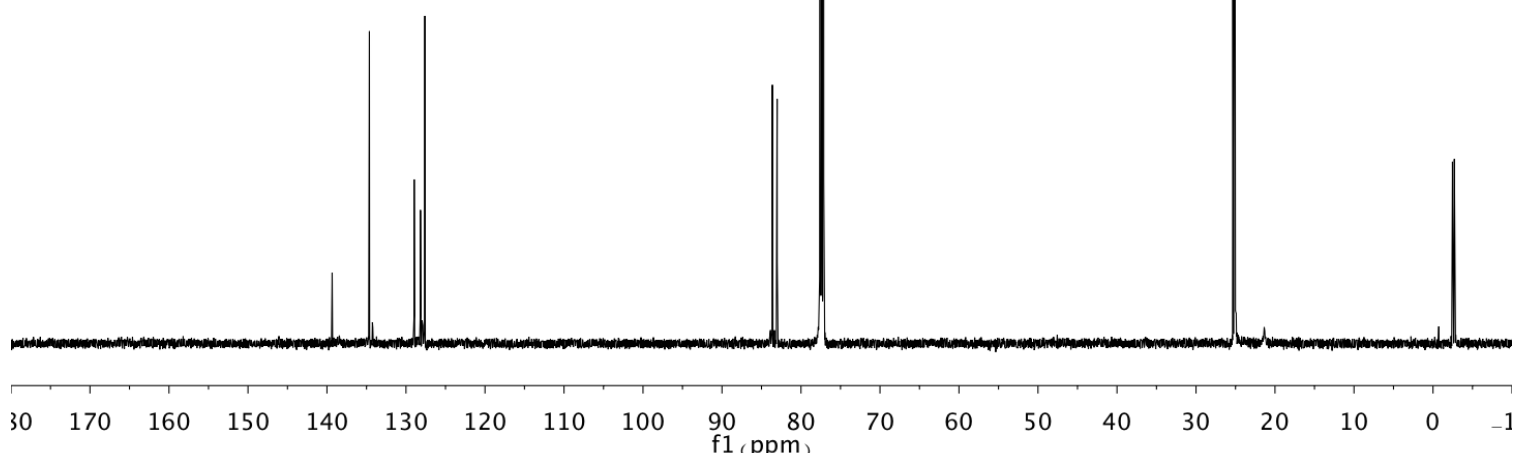

SI-20 

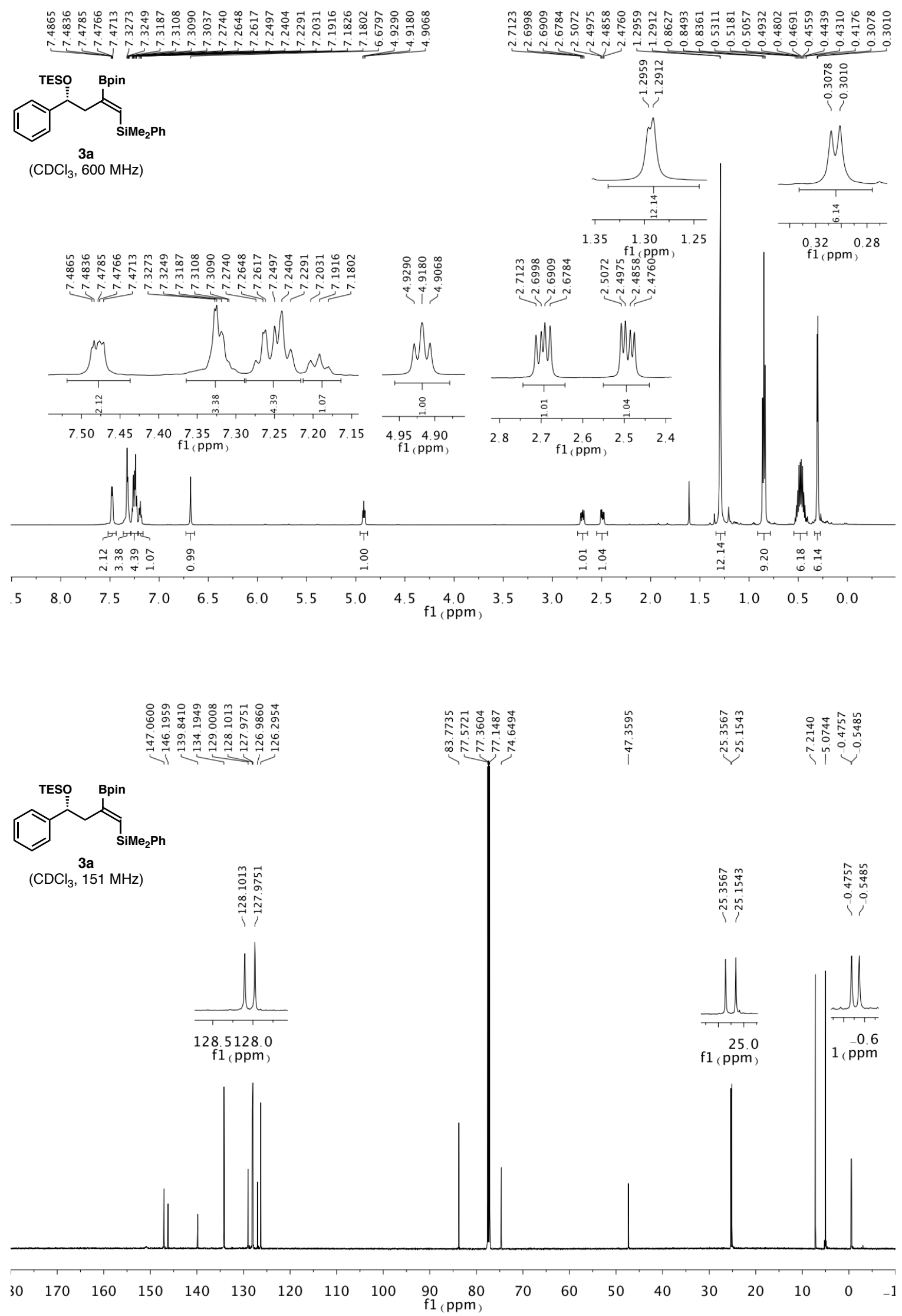

SI-21 

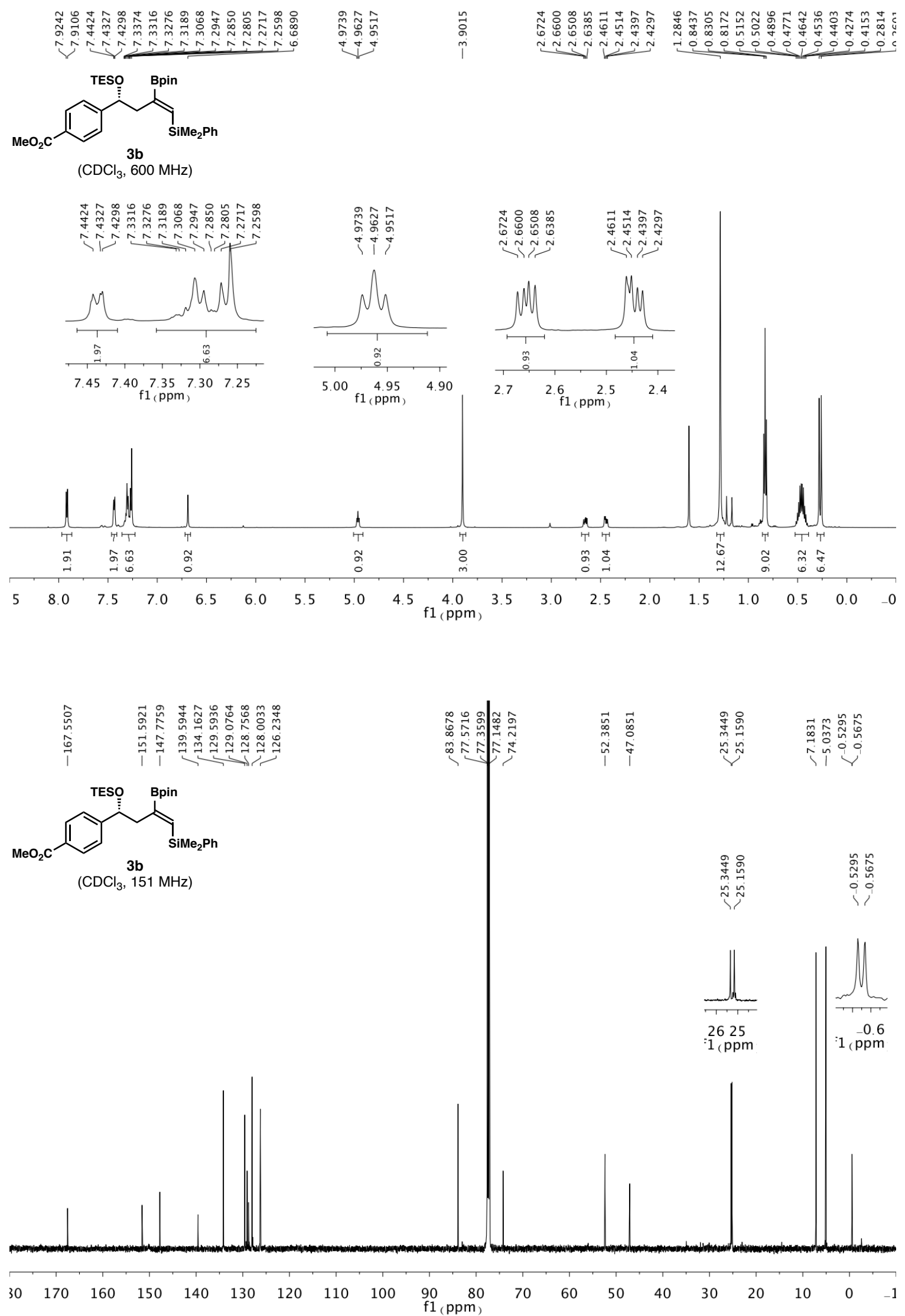

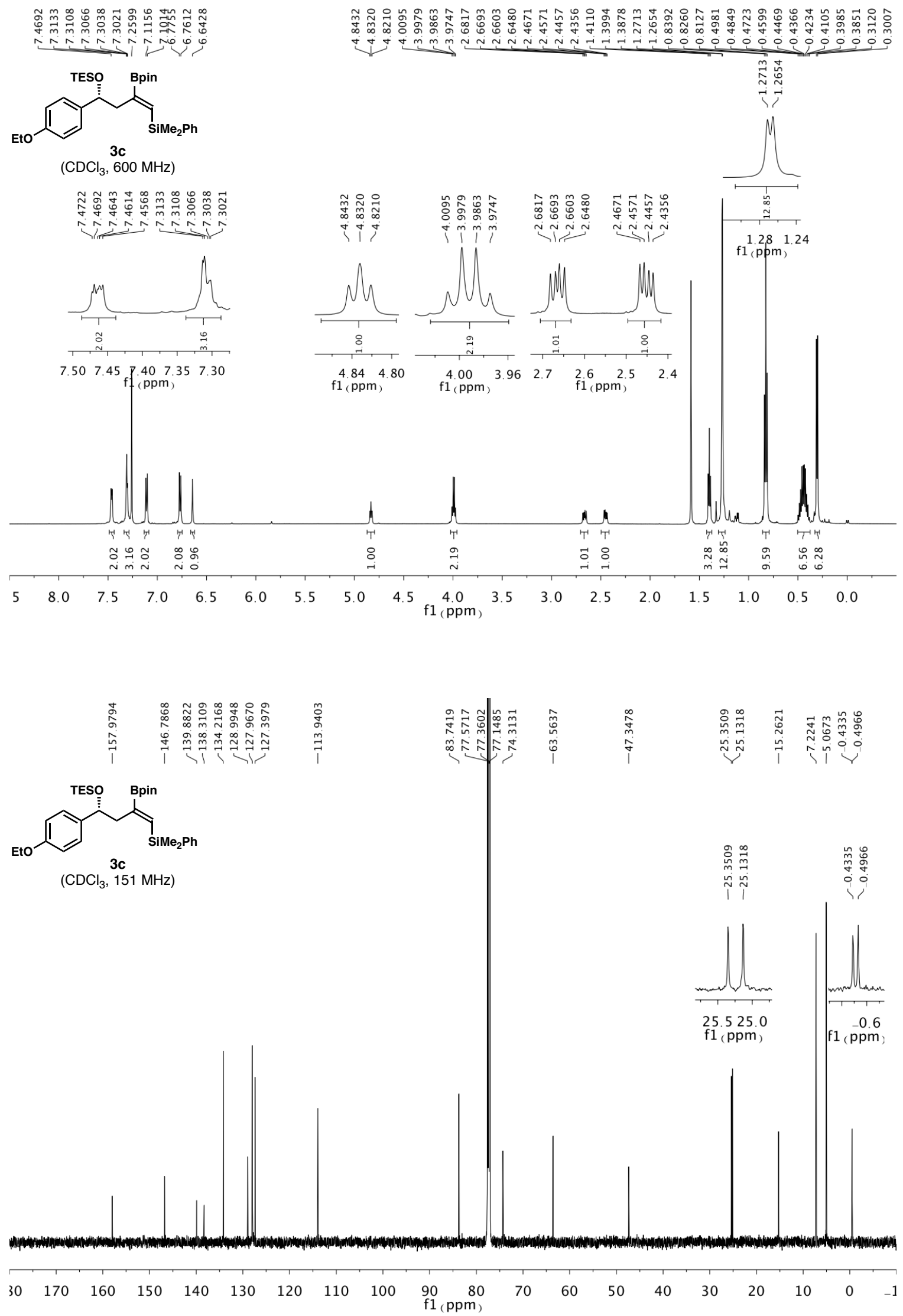

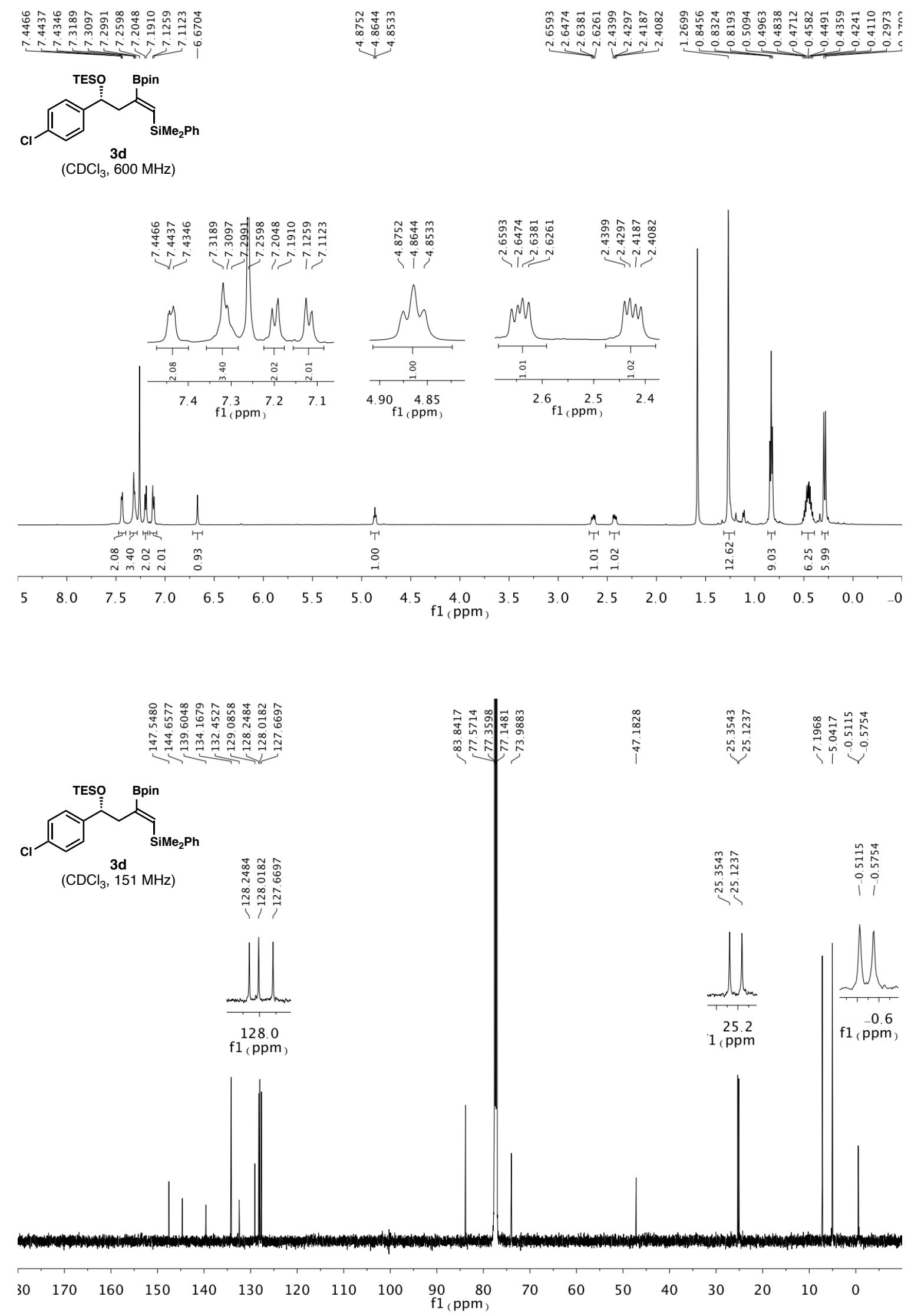

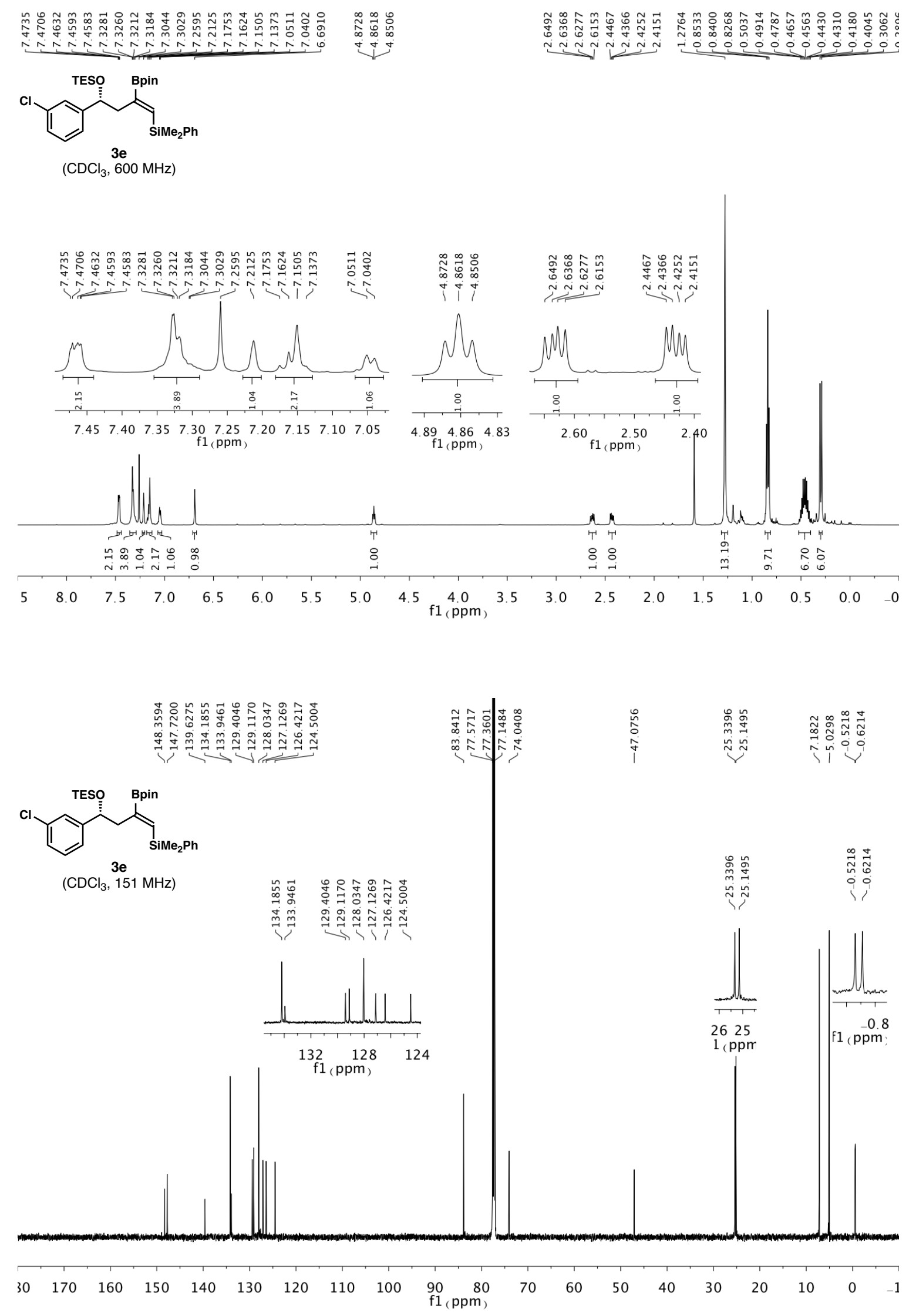

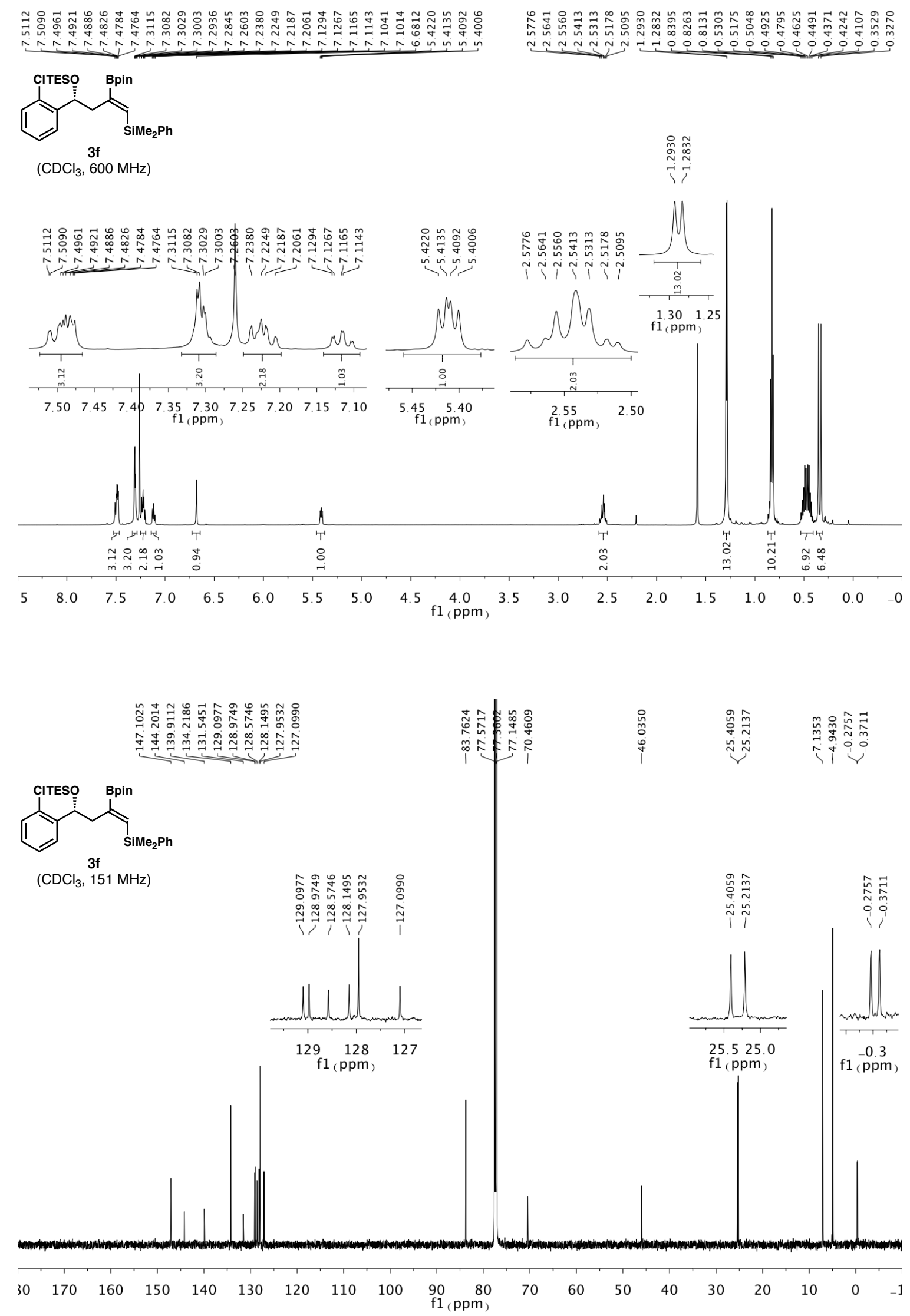

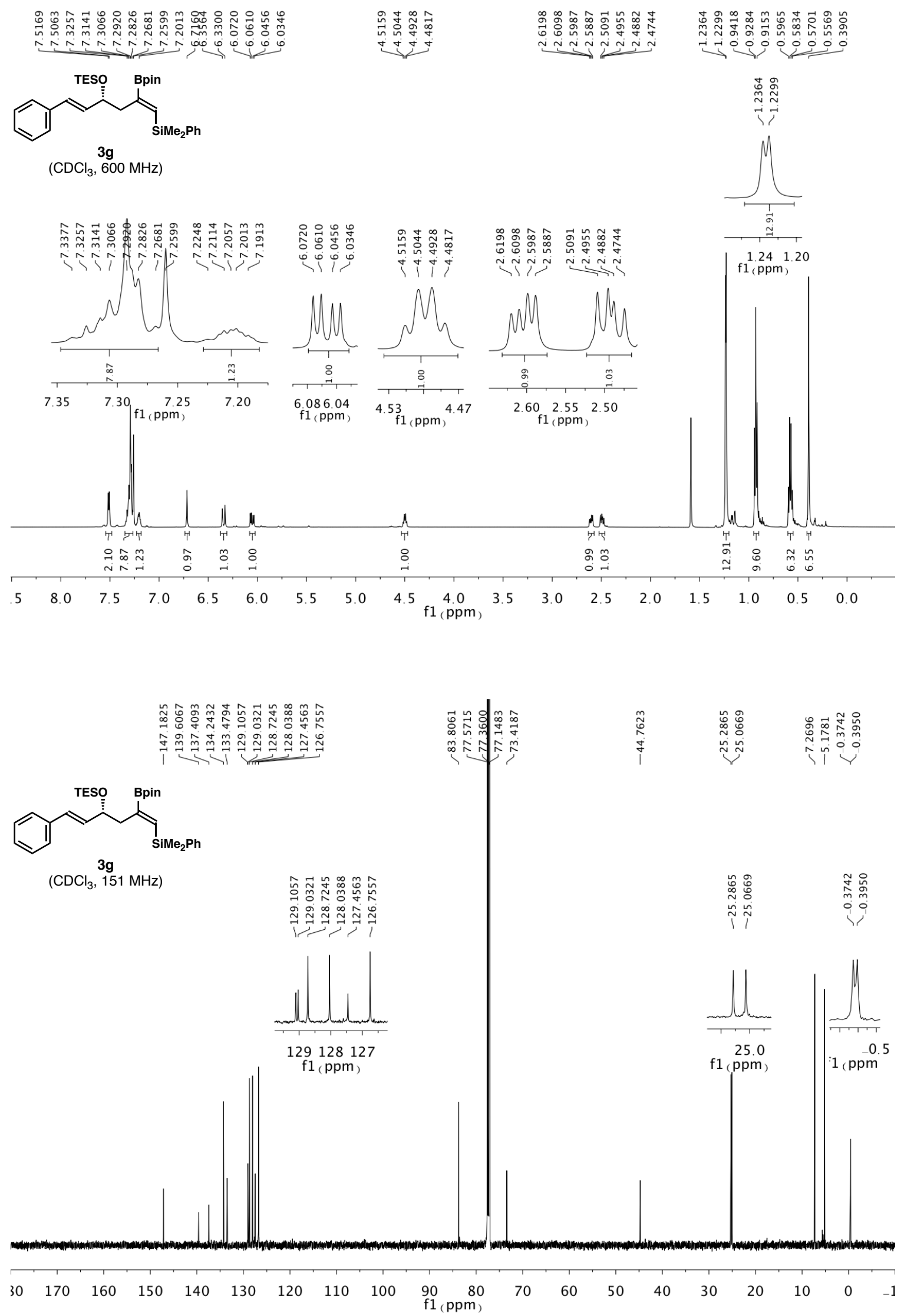

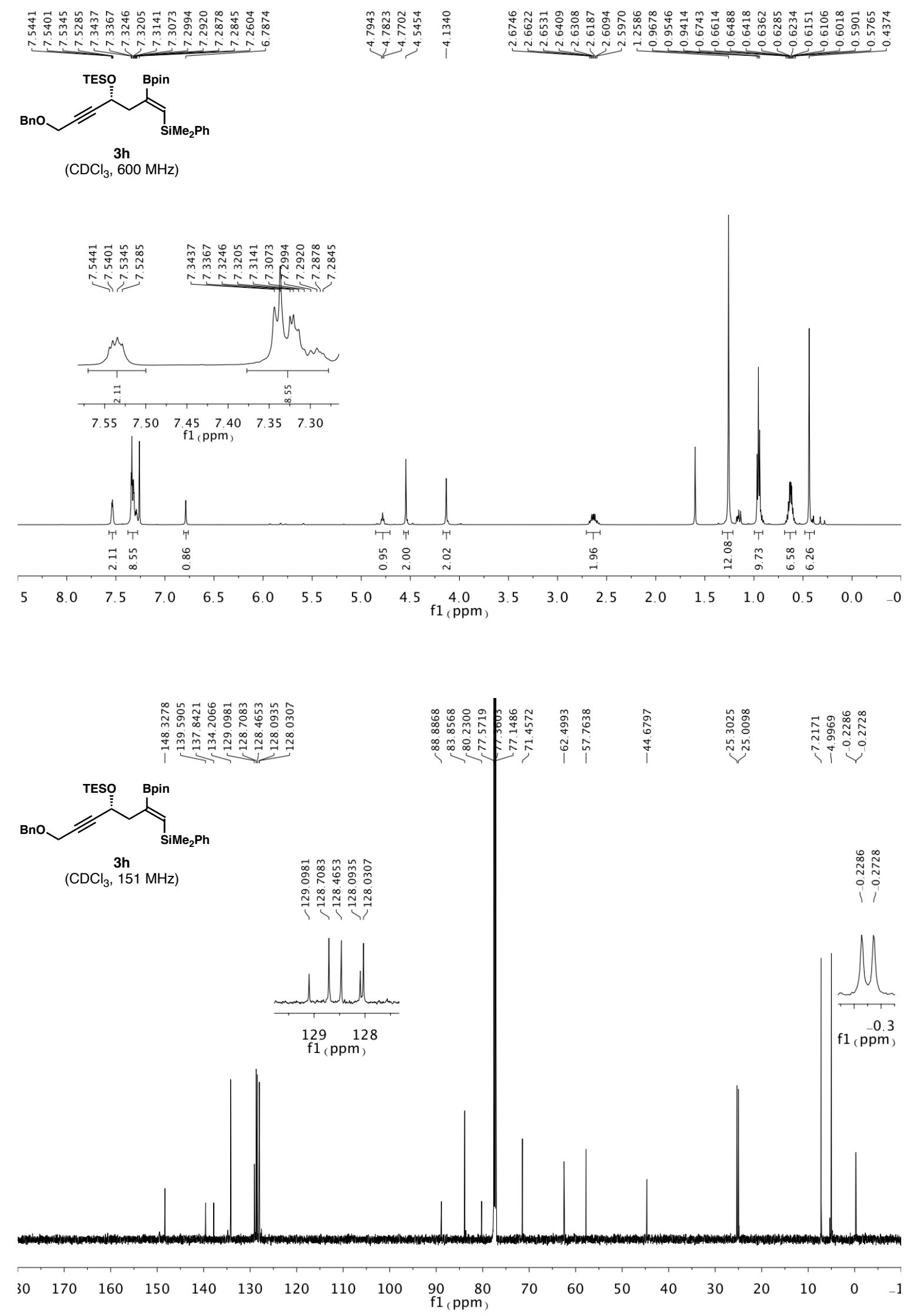


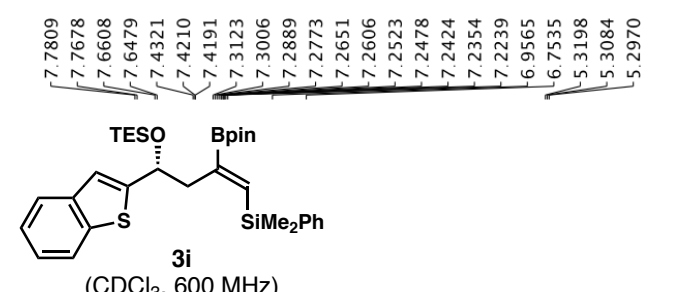

$\left(\mathrm{CDCl}_{3}, 600 \mathrm{MHz}\right)$
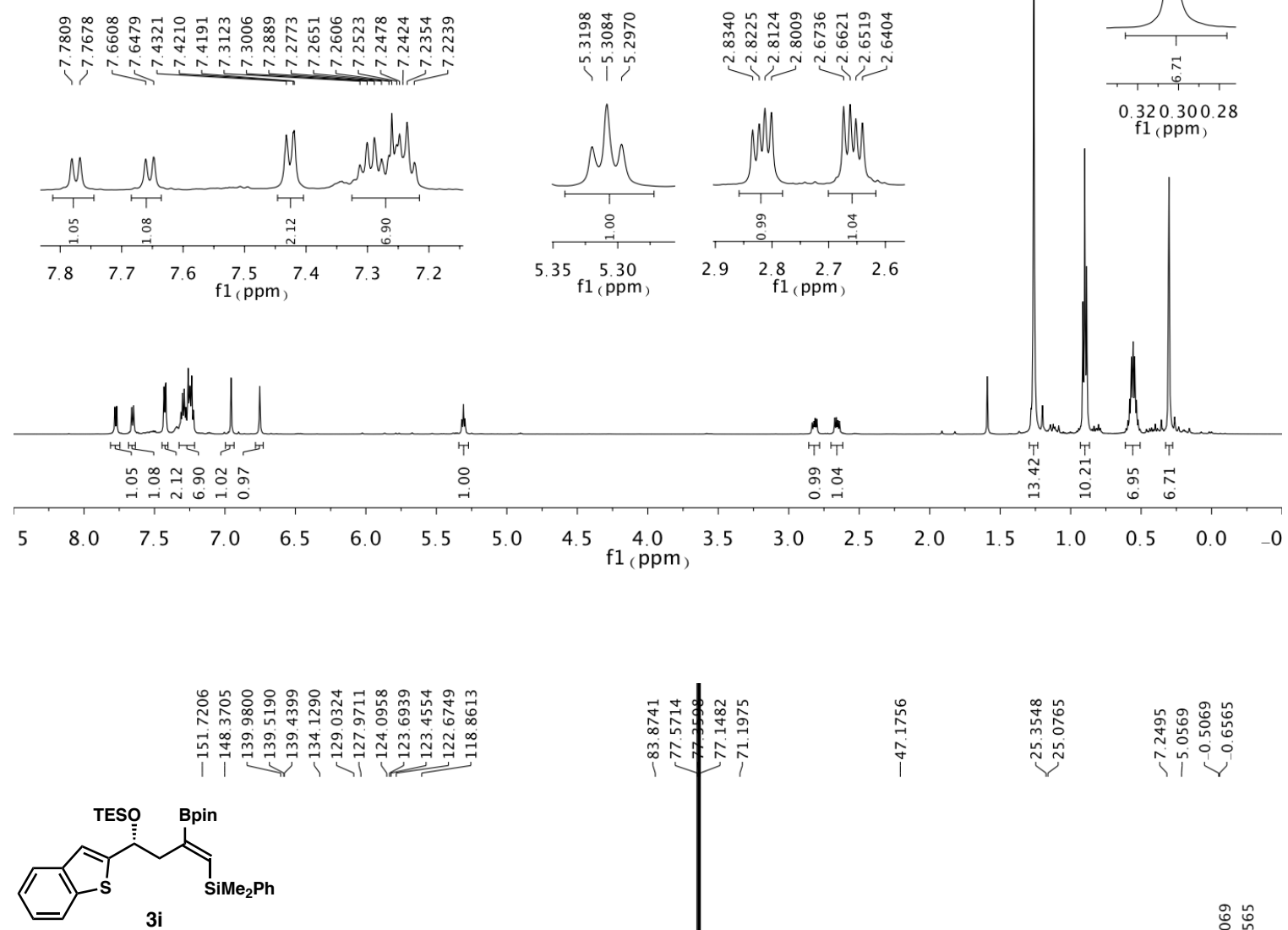

$\left(\mathrm{CDCl}_{3}, 151 \mathrm{MHz}\right)$

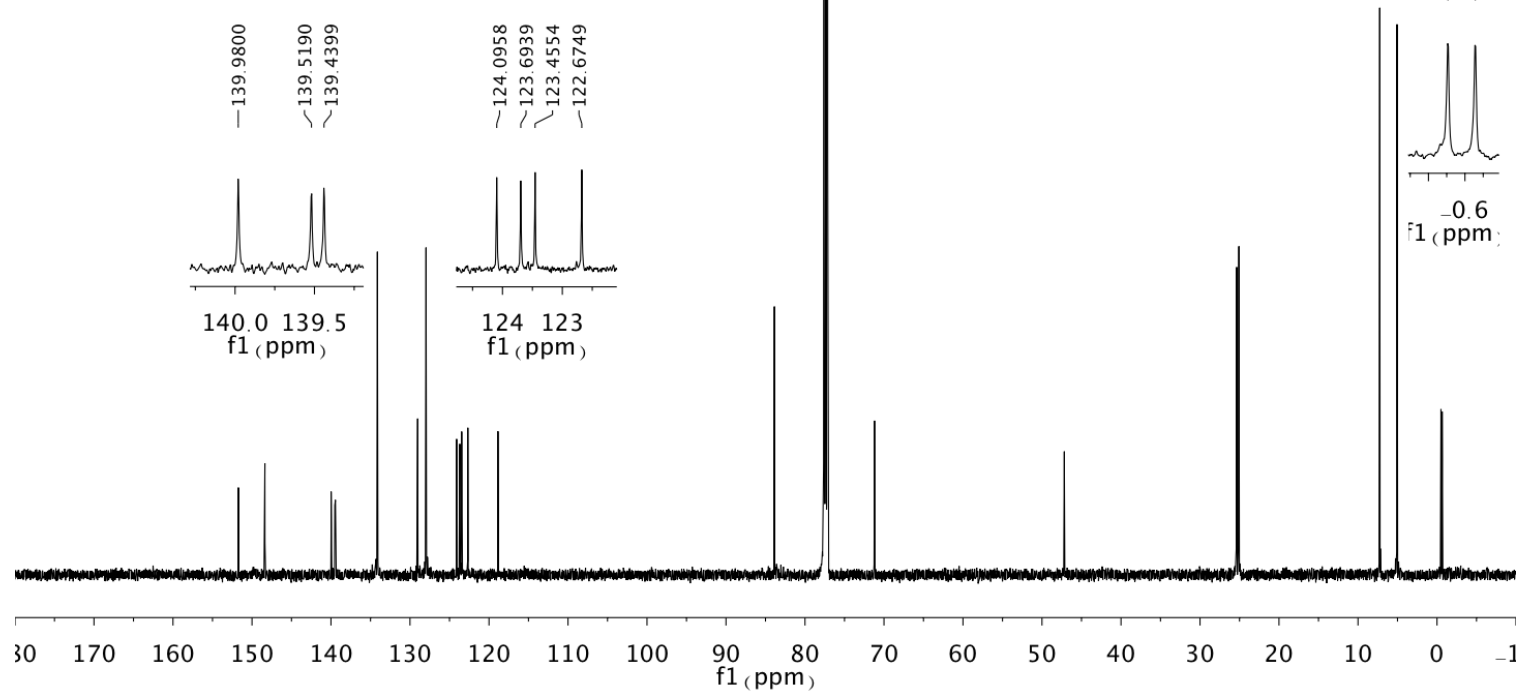



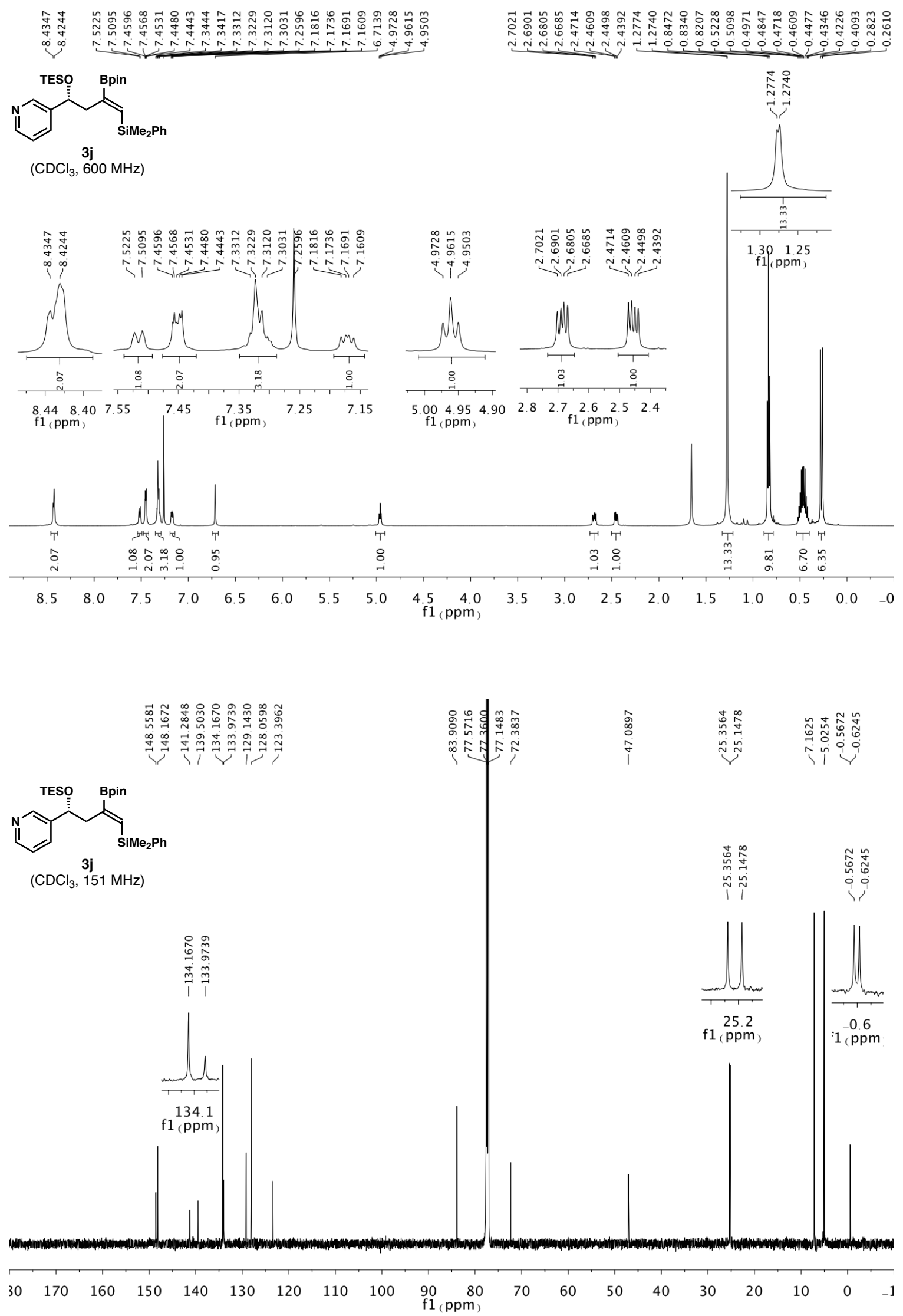


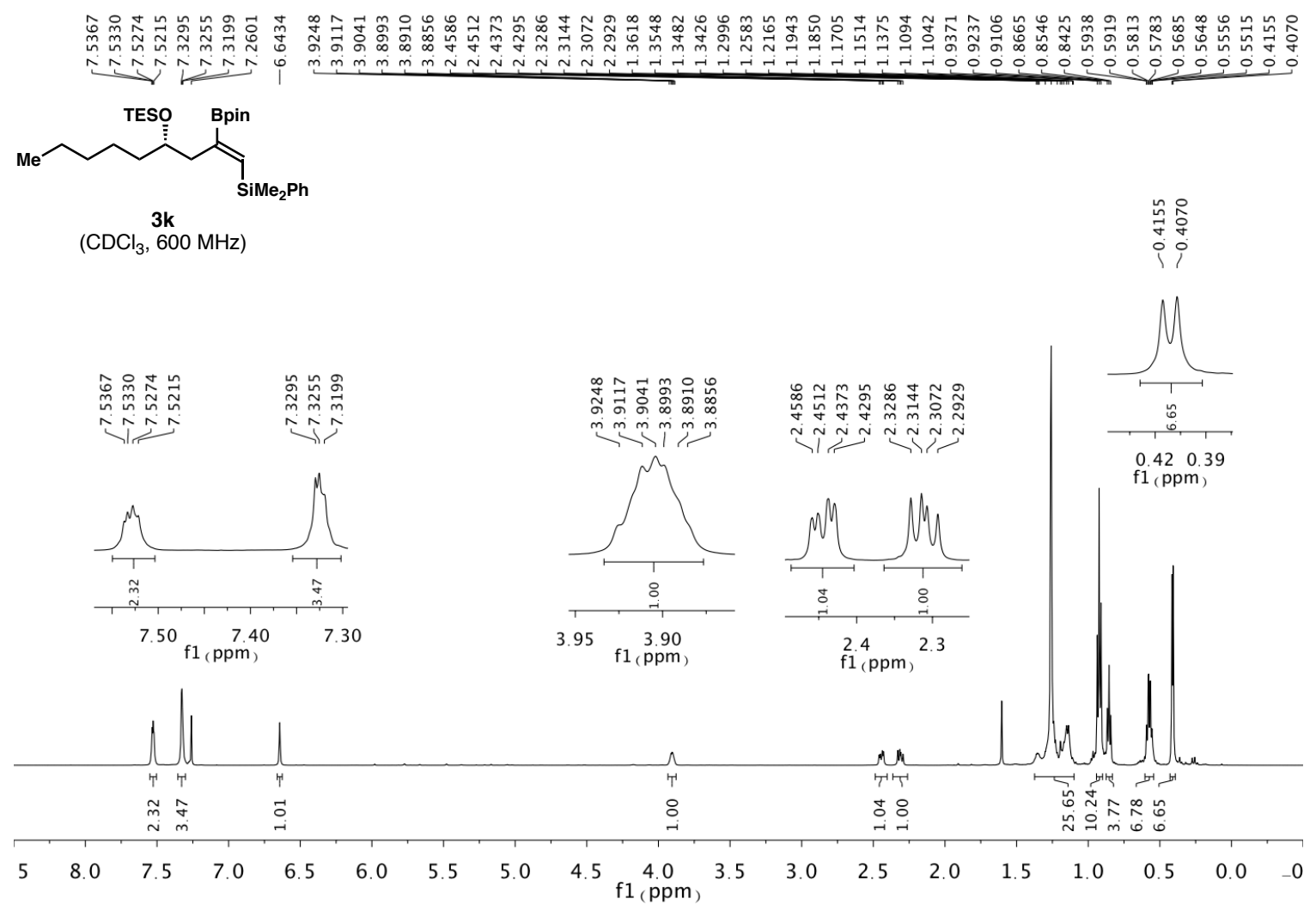

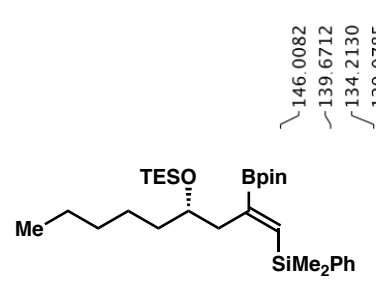

$\mathbf{3 k}$
$\left(\mathrm{CDCl}_{3}, 151 \mathrm{MHz}\right)$

$\mathrm{f} 1$ (ppm) 


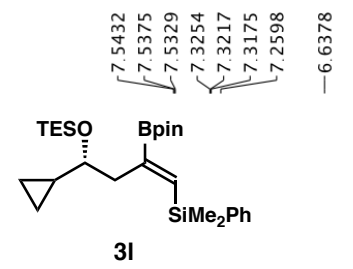

$\left(\mathrm{CDCl}_{3}, 600 \mathrm{MHz}\right)$

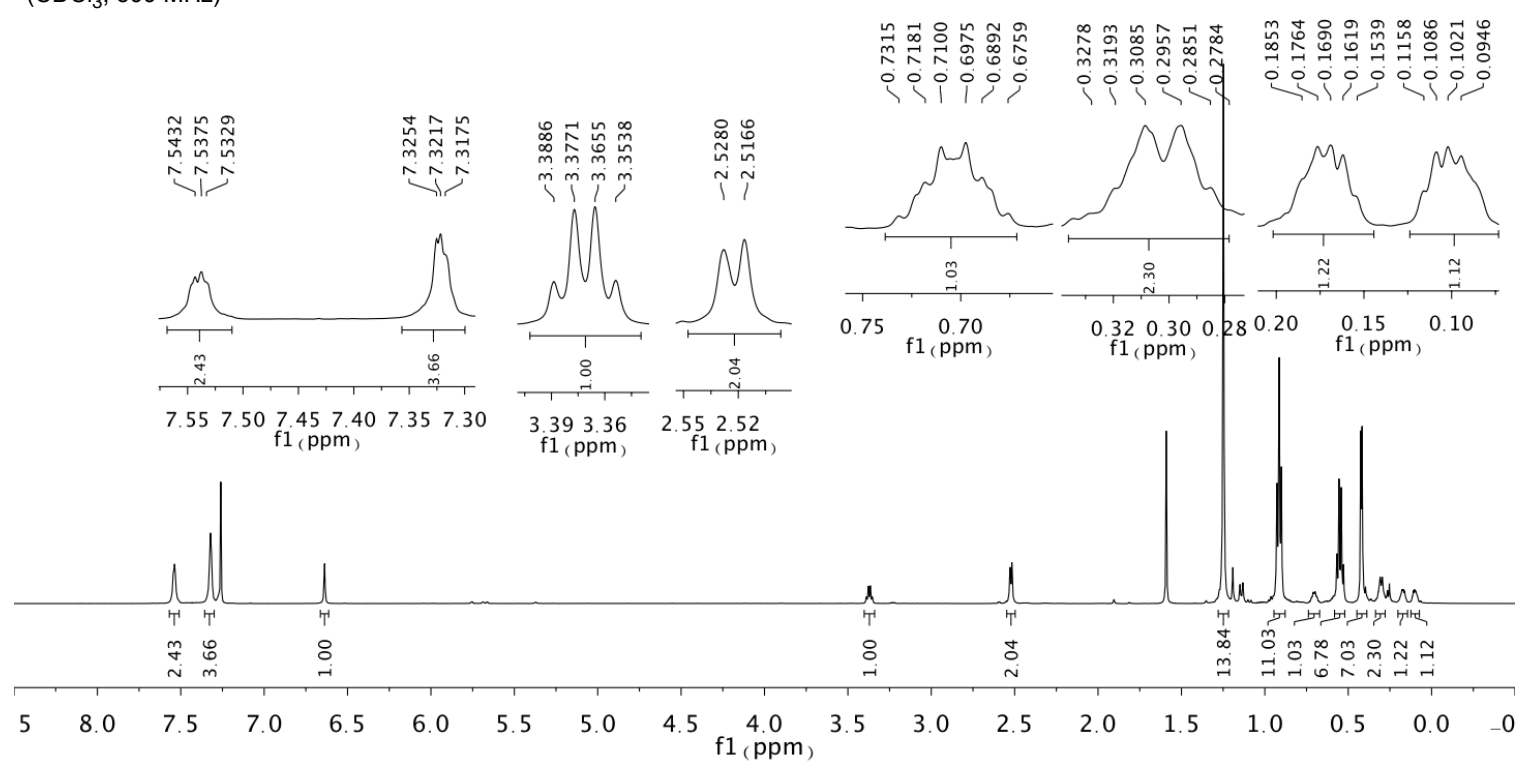

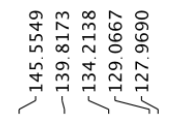

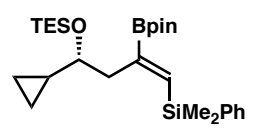

3I

$\left(\mathrm{CDCl}_{3}, 151 \mathrm{MHz}\right)$

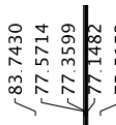

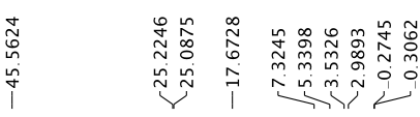

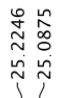
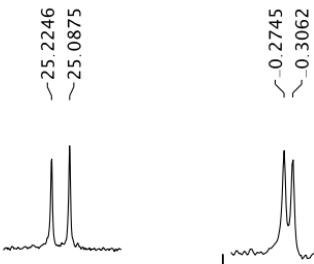

25.224 .8

$\mathrm{f1}(\mathrm{ppm})$

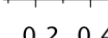

$-0.2-0.4$

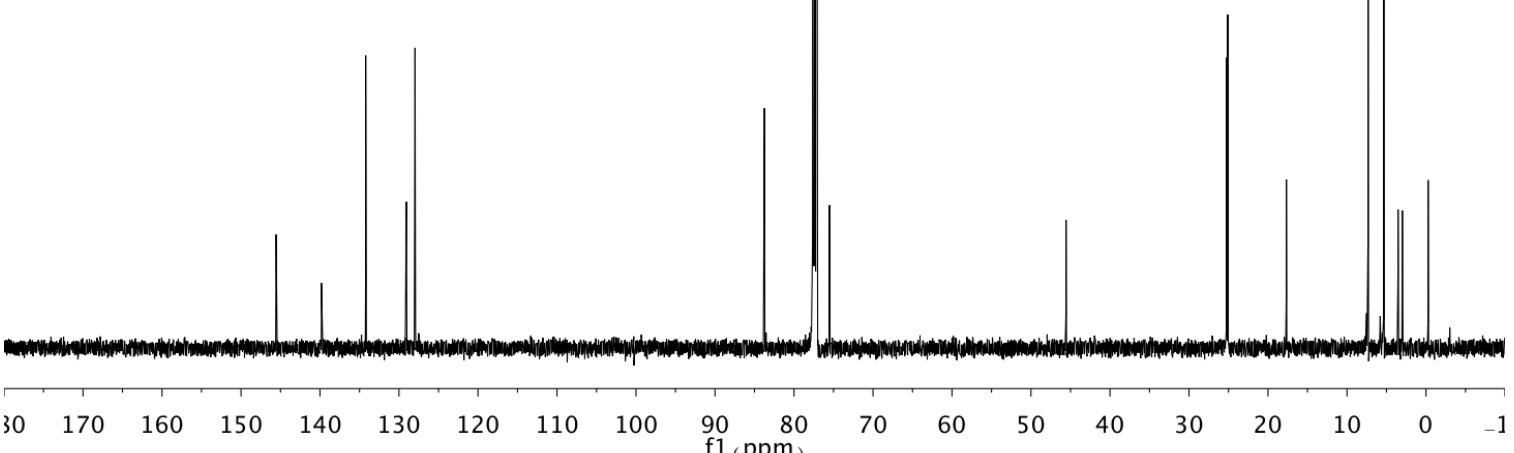

SI-32 

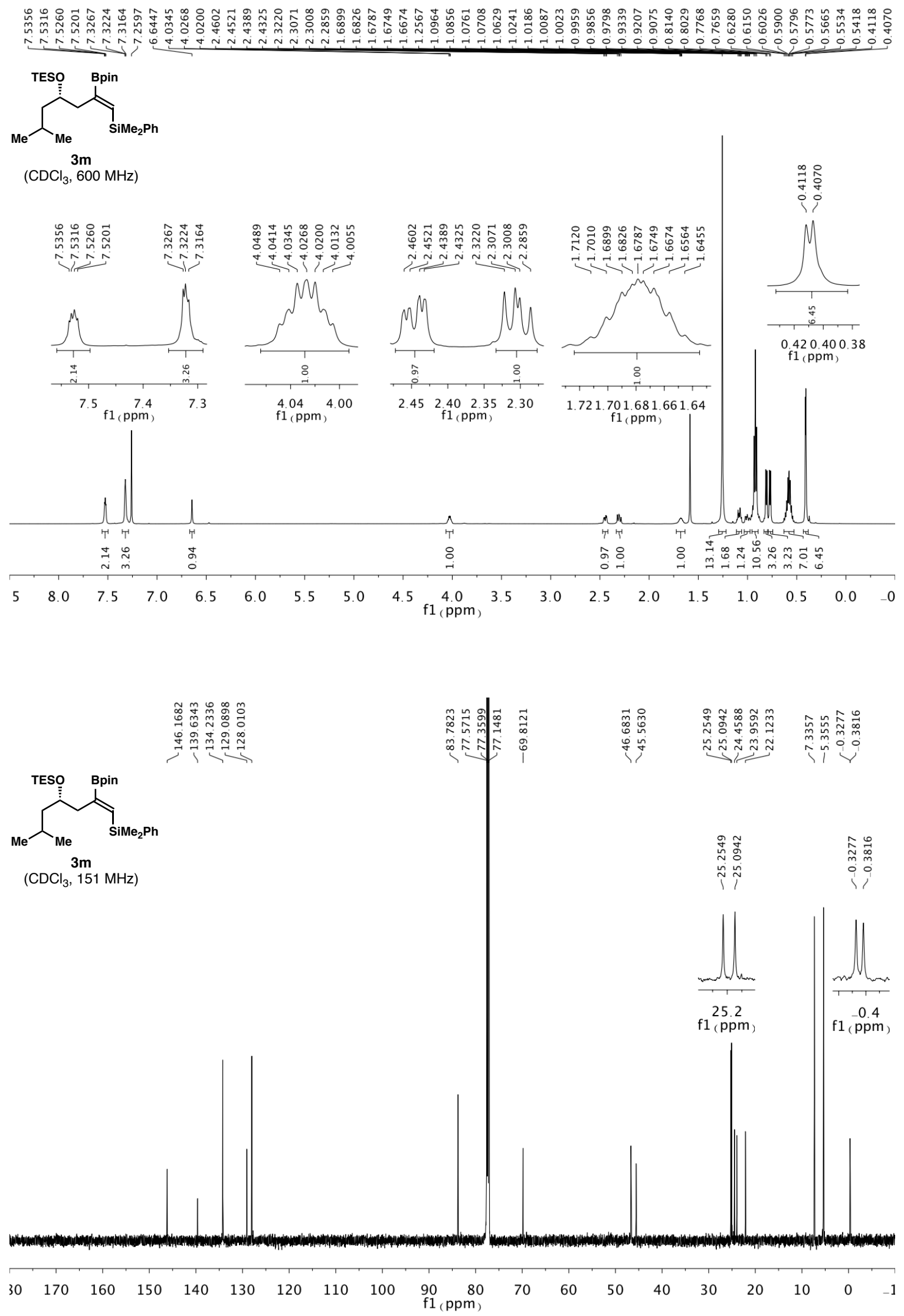

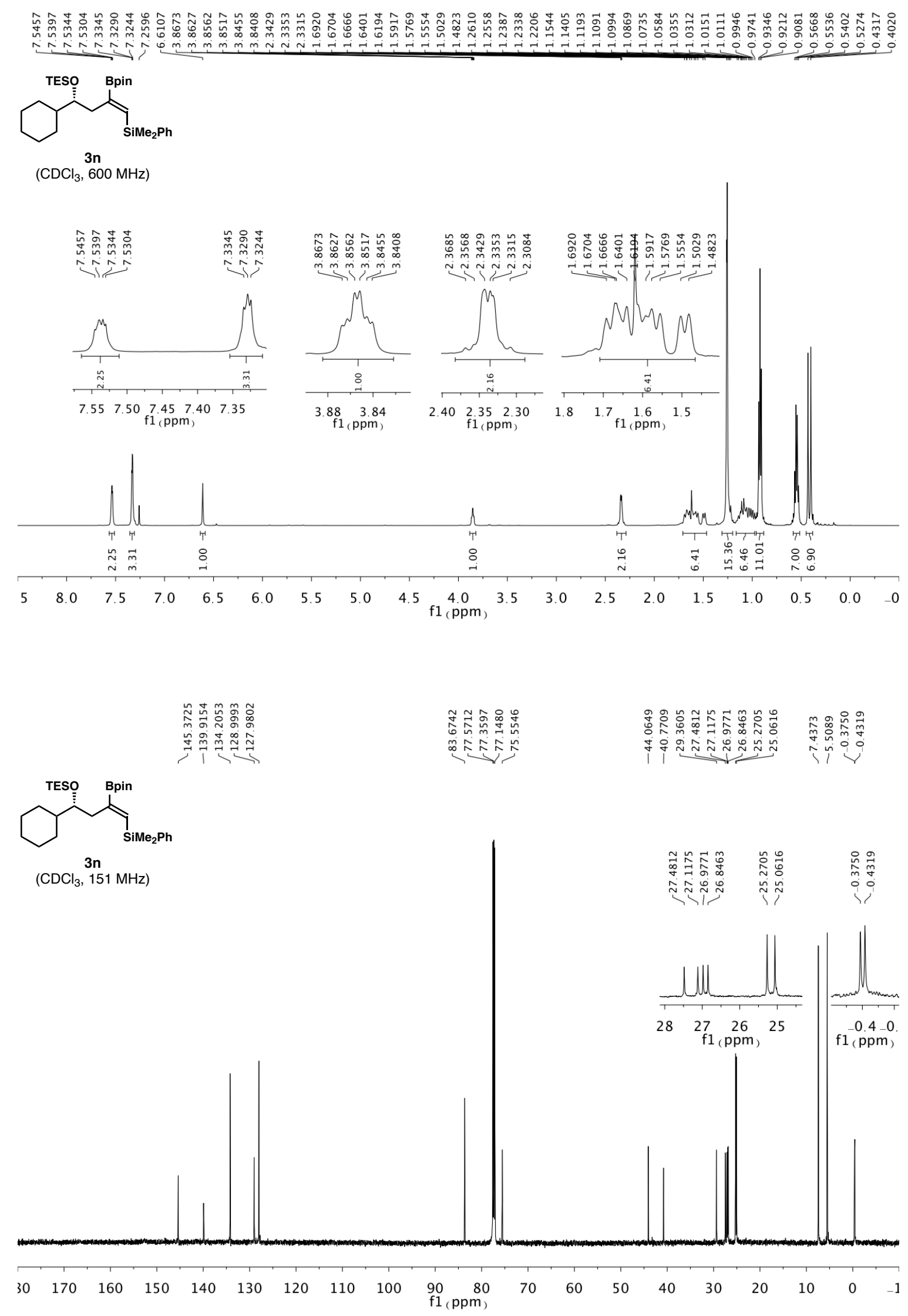

SI-34 

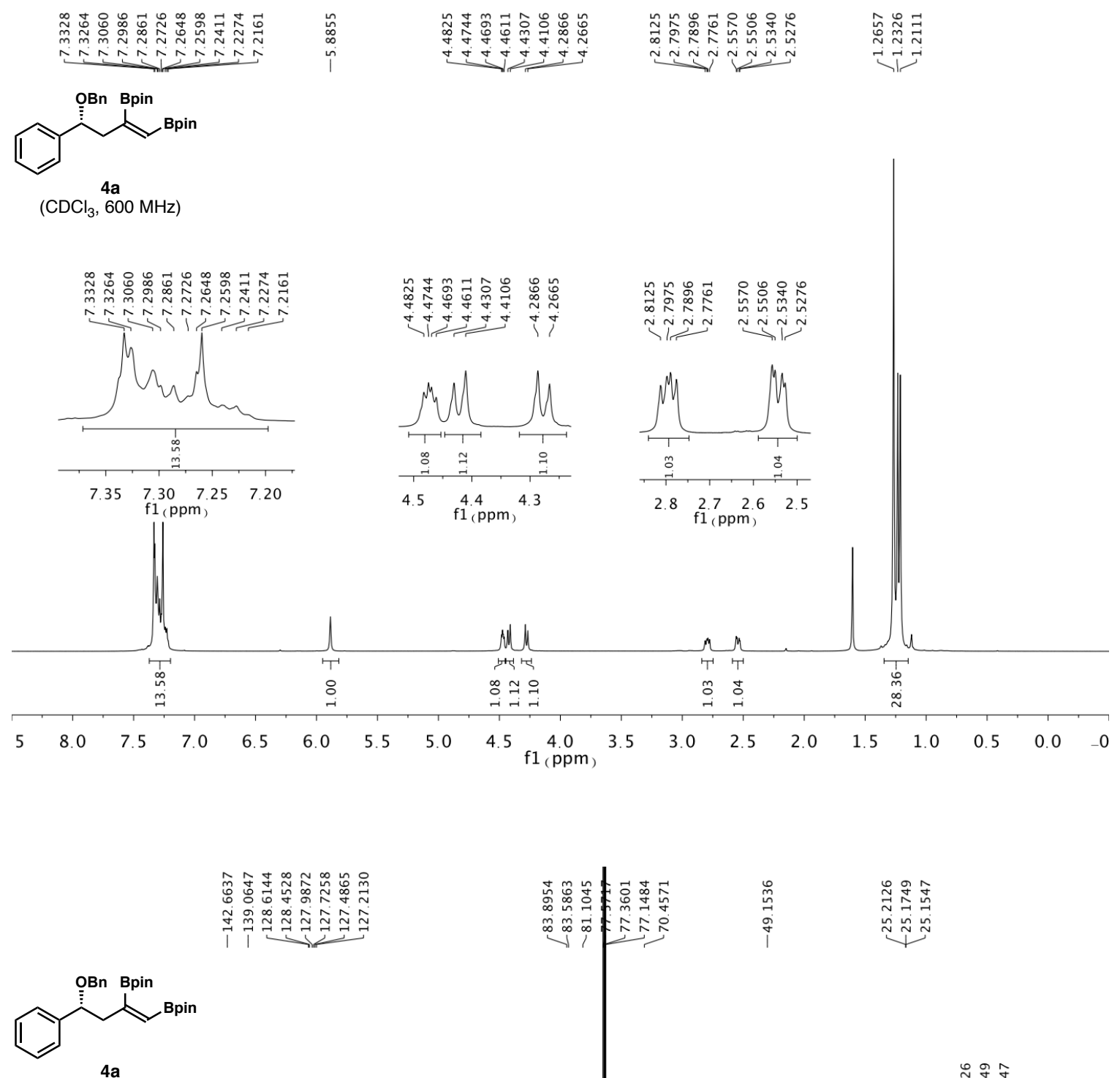

$\left(\mathrm{CDCl}_{3}, 151 \mathrm{MHz}\right)$

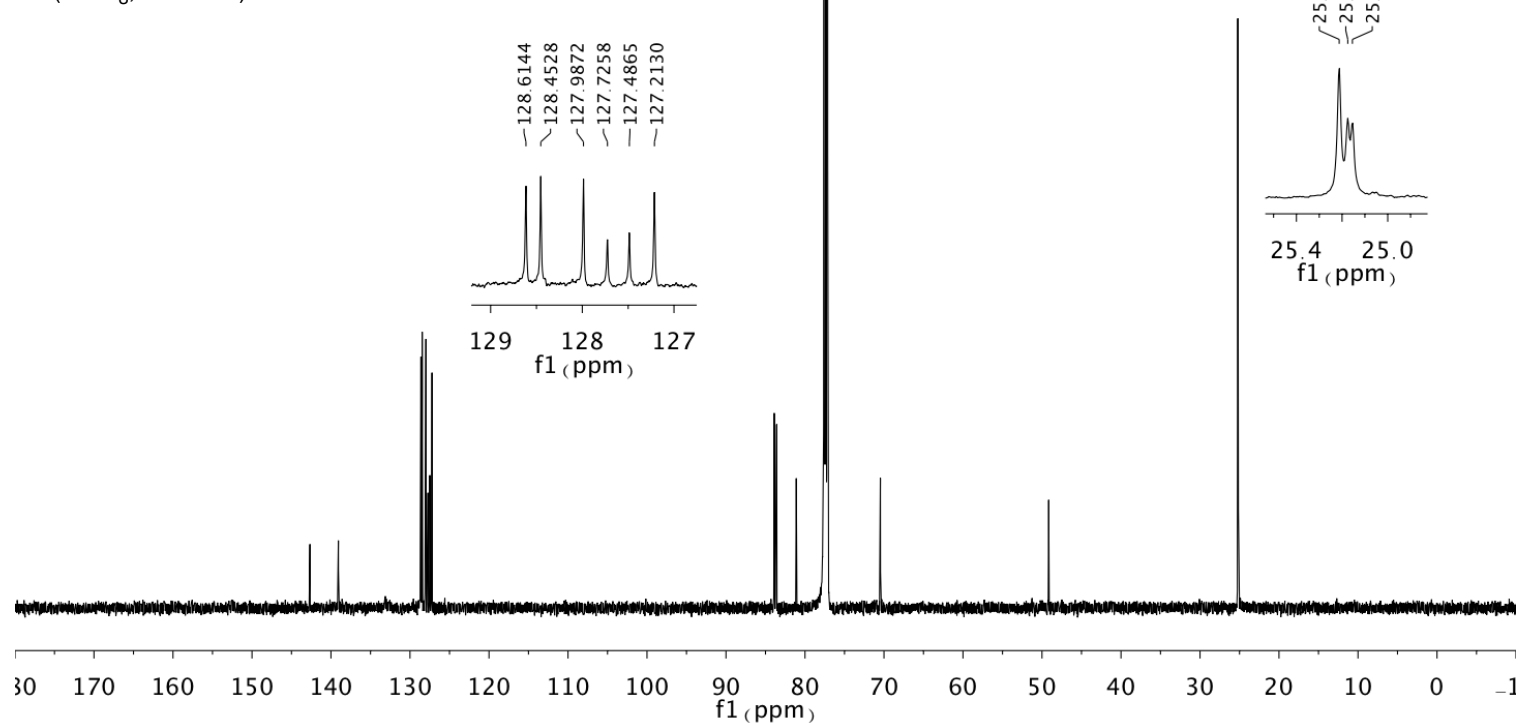



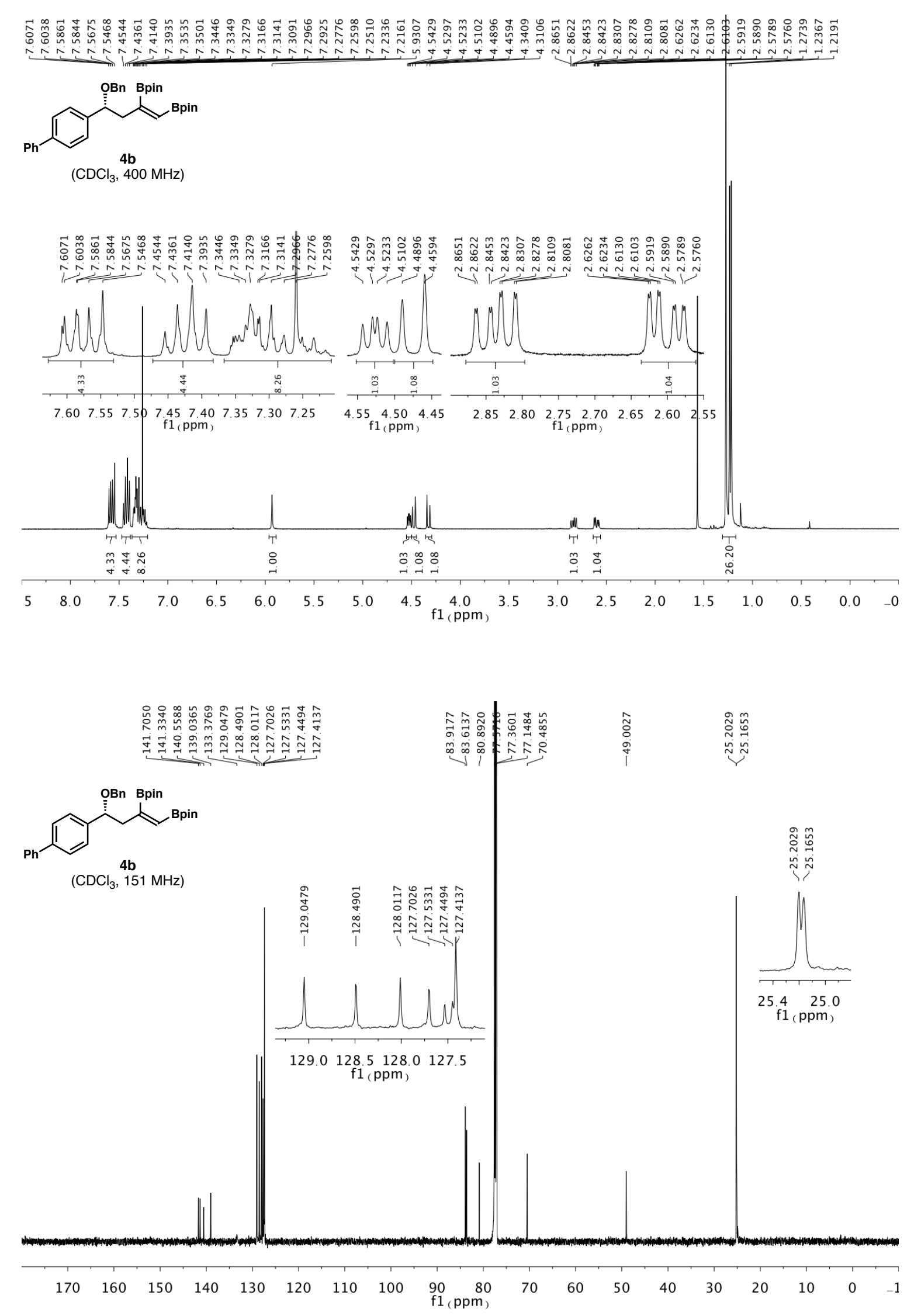

SI-36 

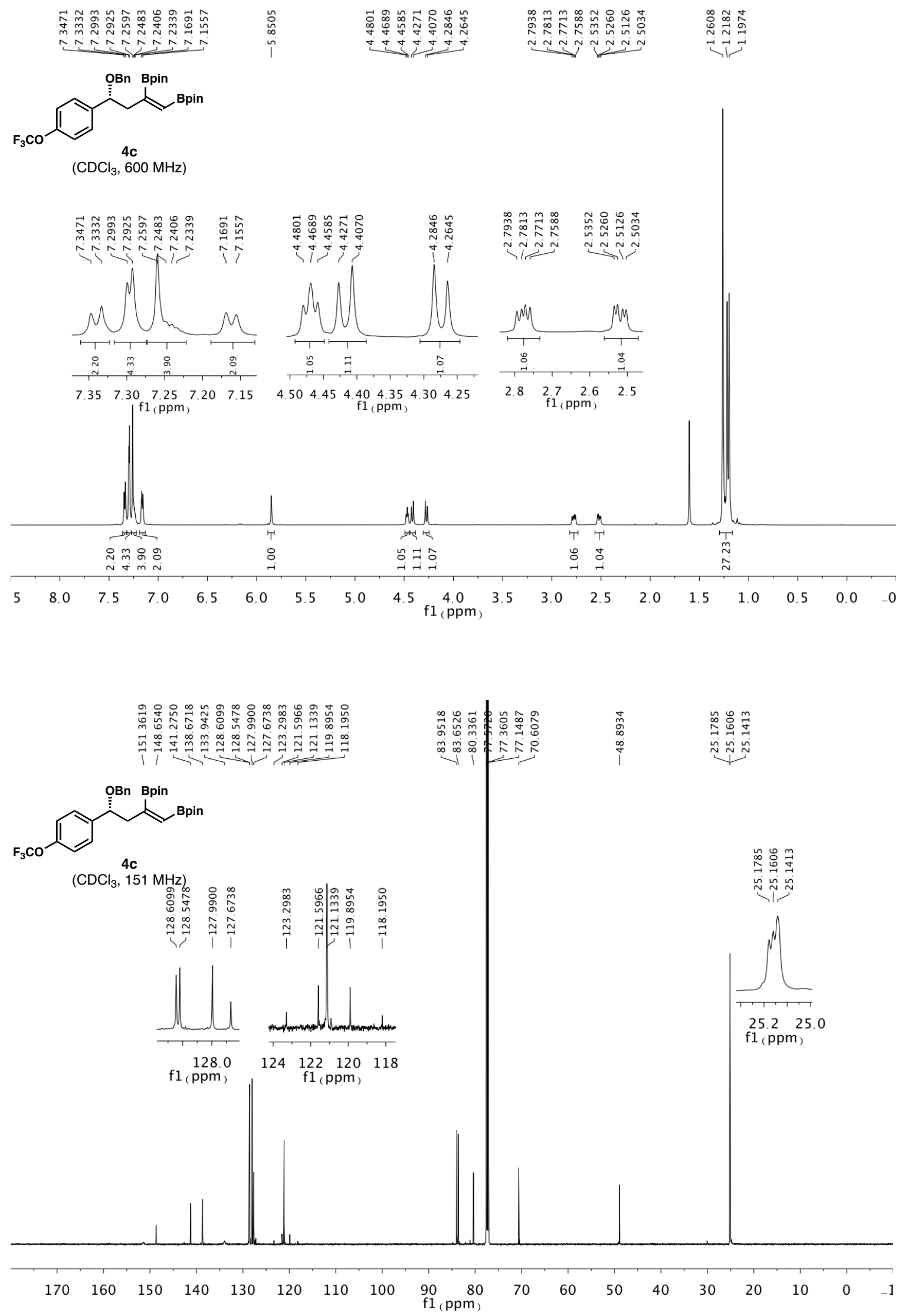

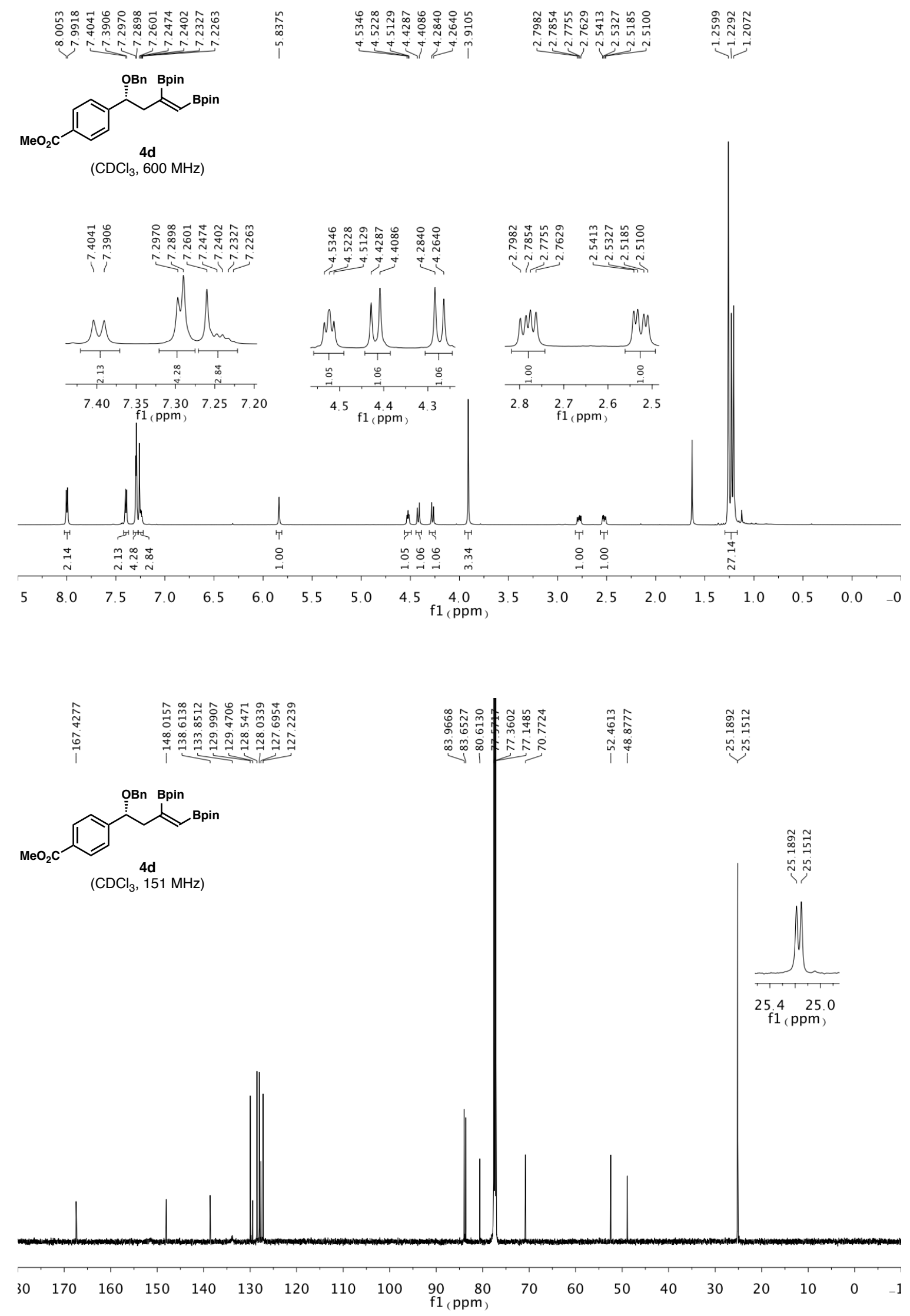

SI-38 

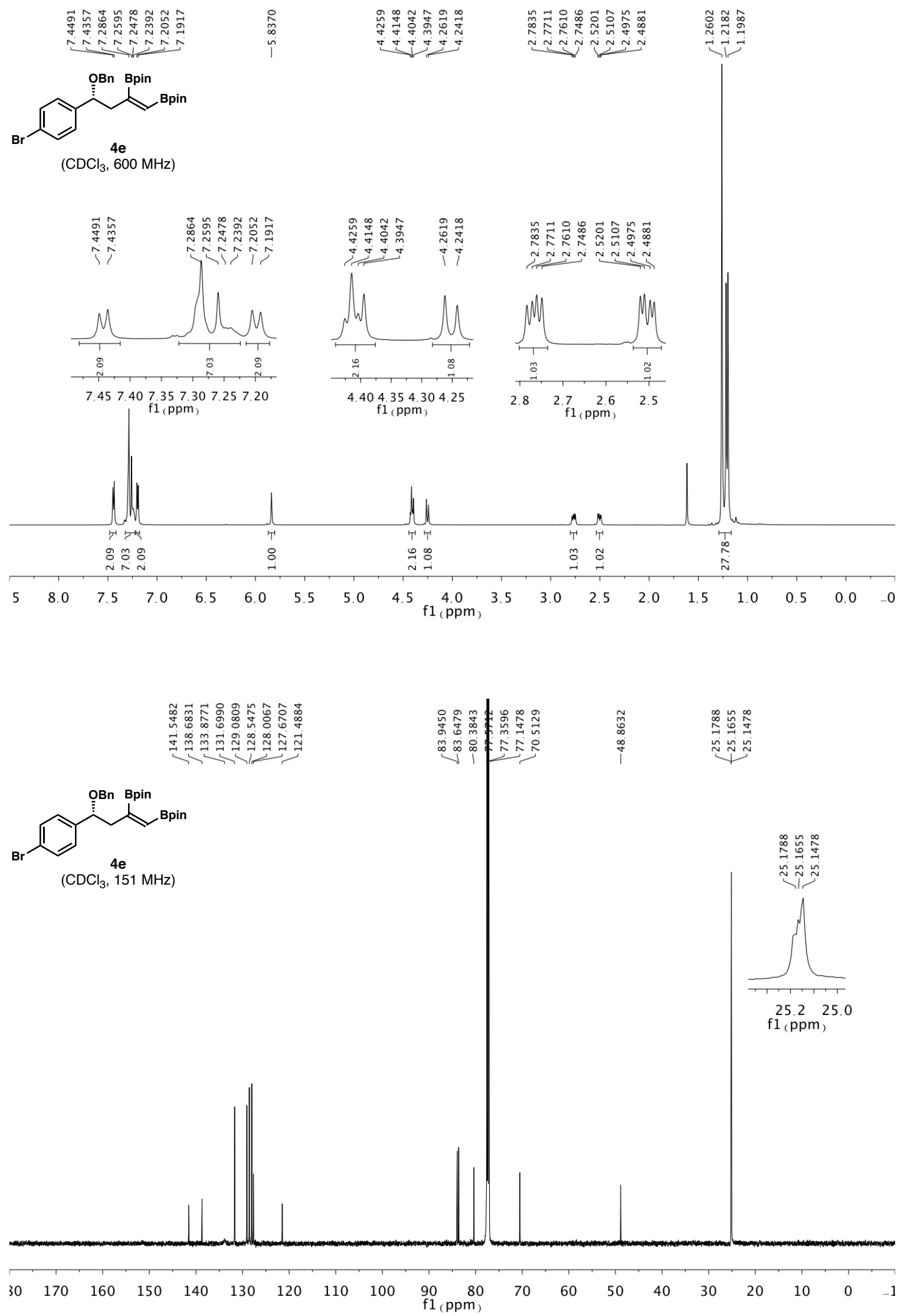

SI-39 

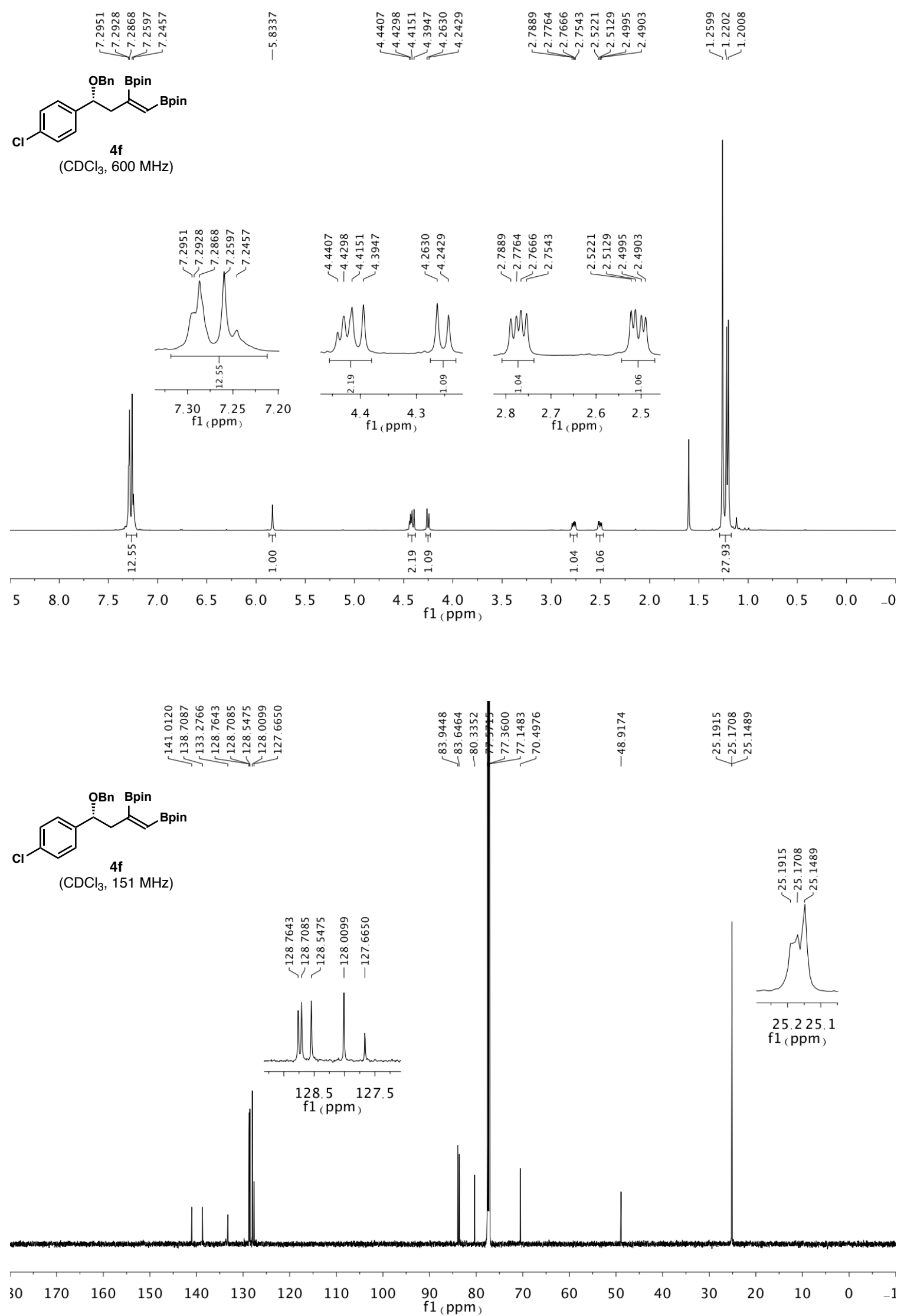

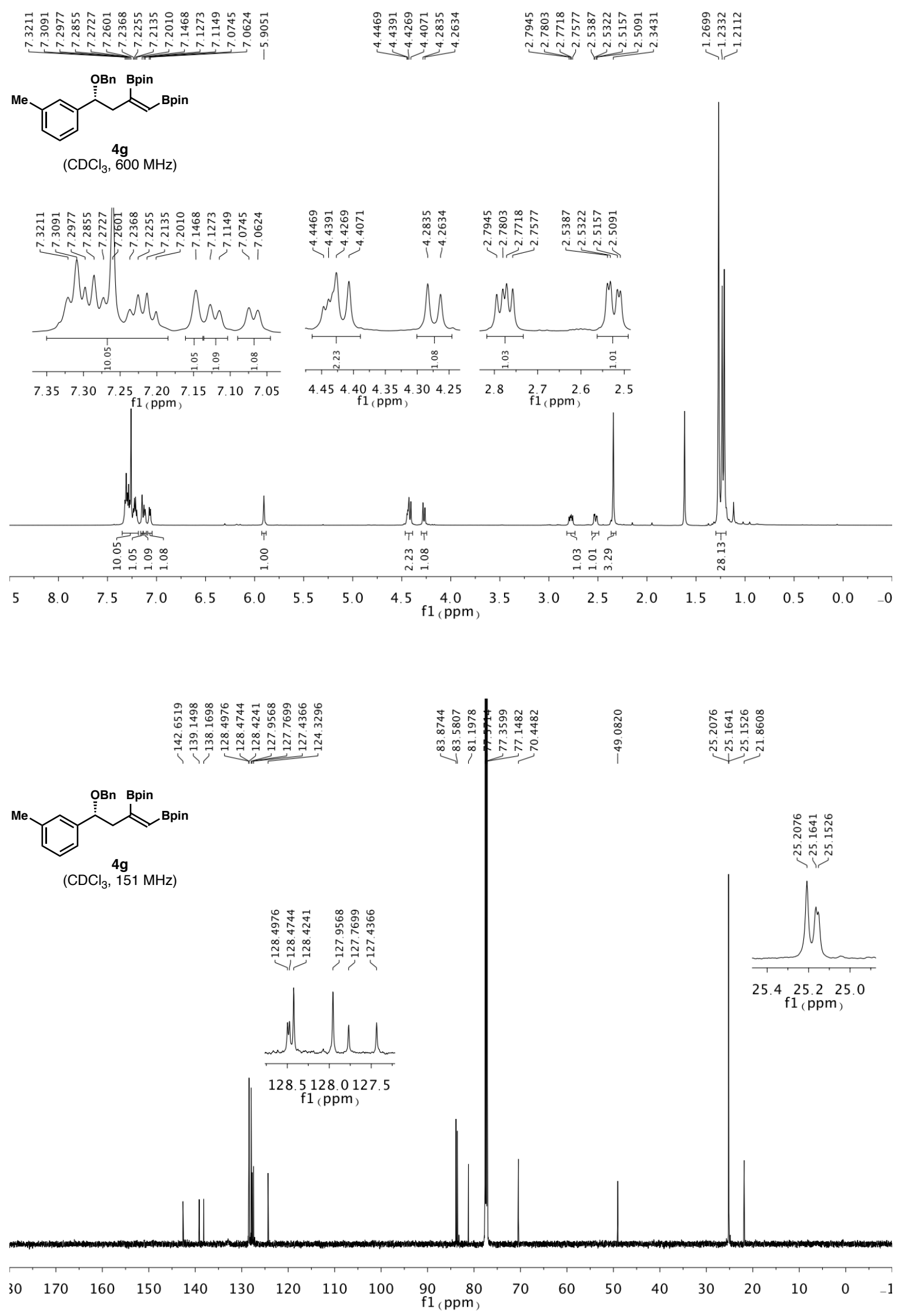

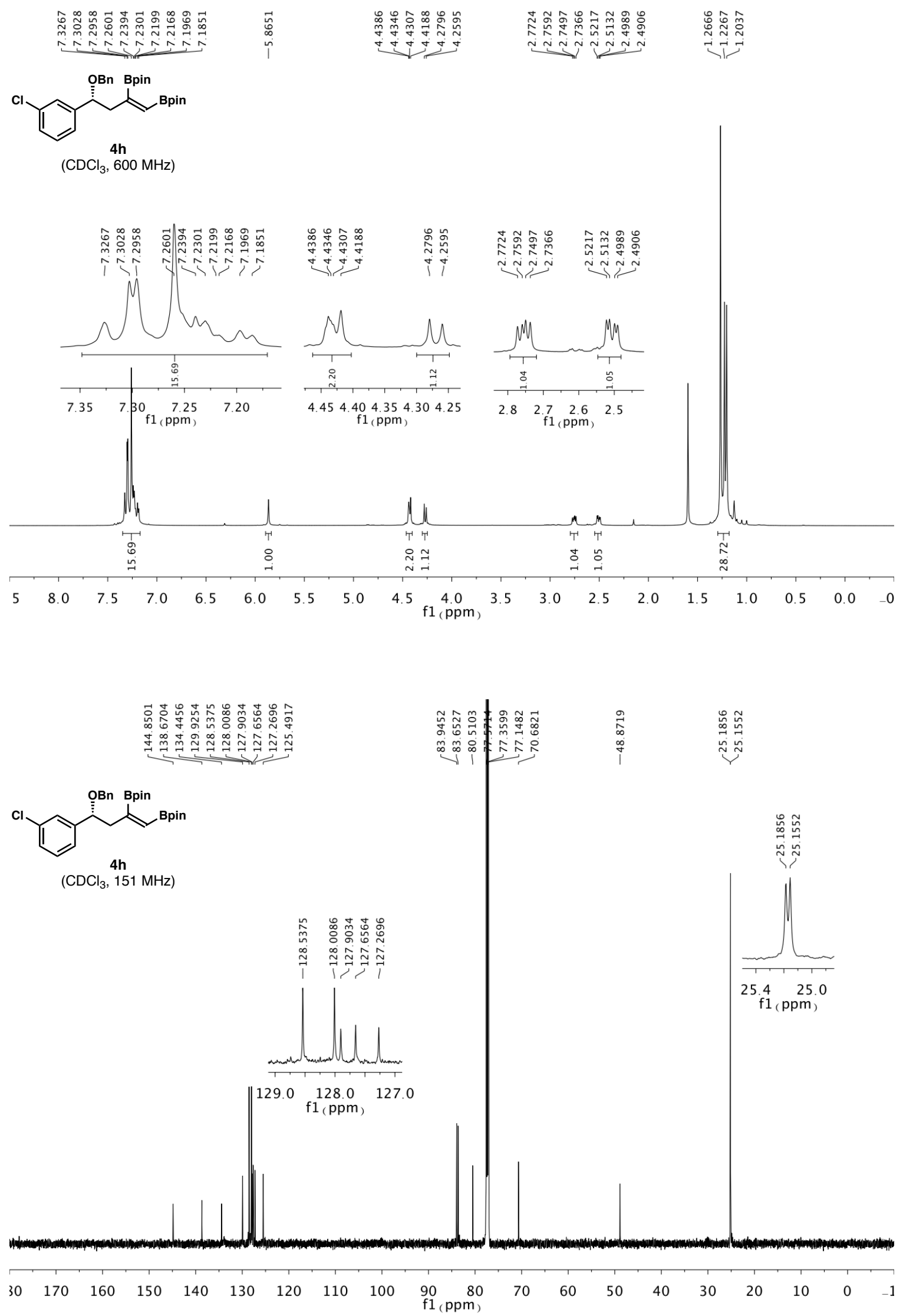

SI-42 

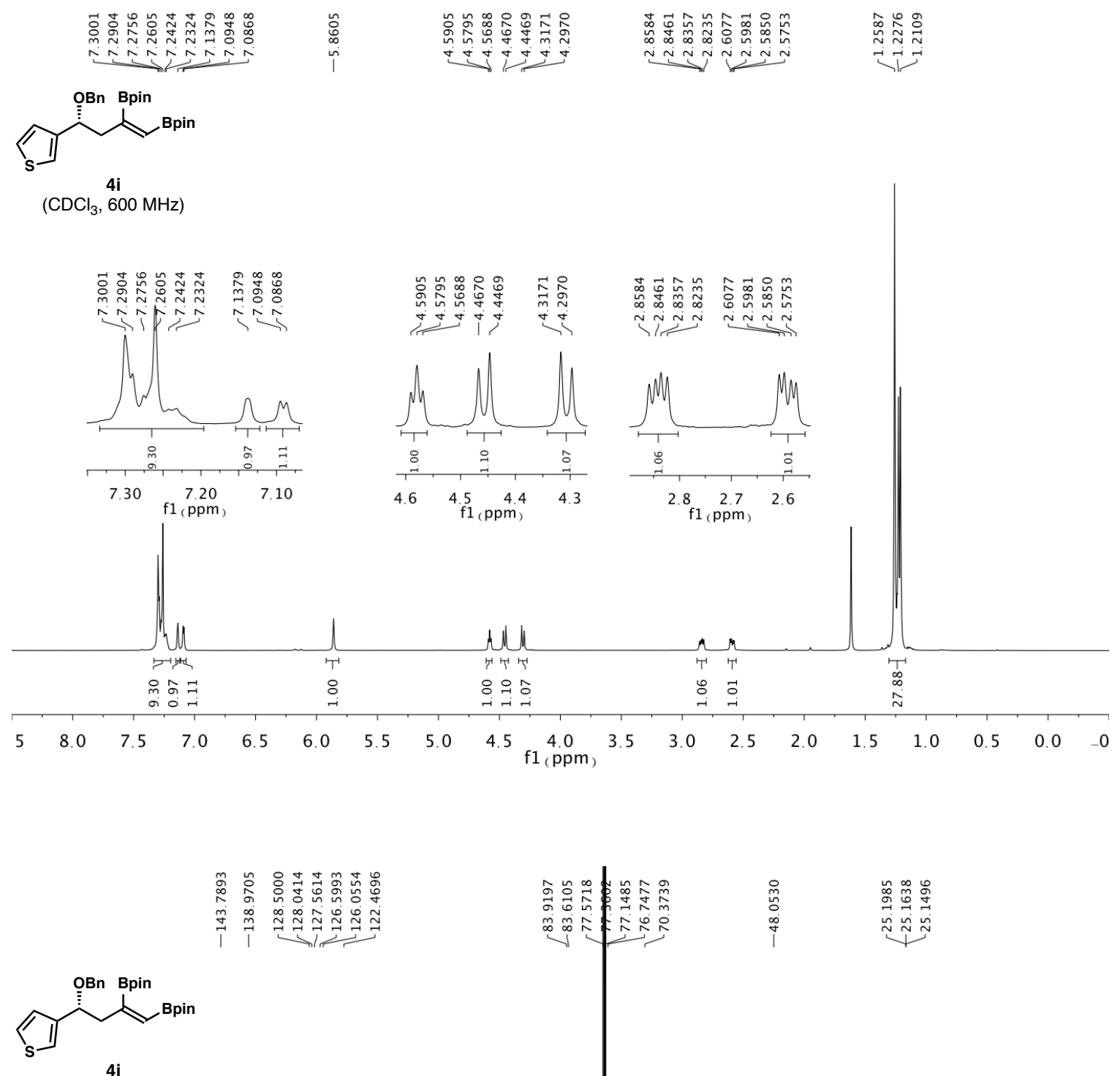

$\left(\mathrm{CDCl}_{3}, 151 \mathrm{MHz}\right)$

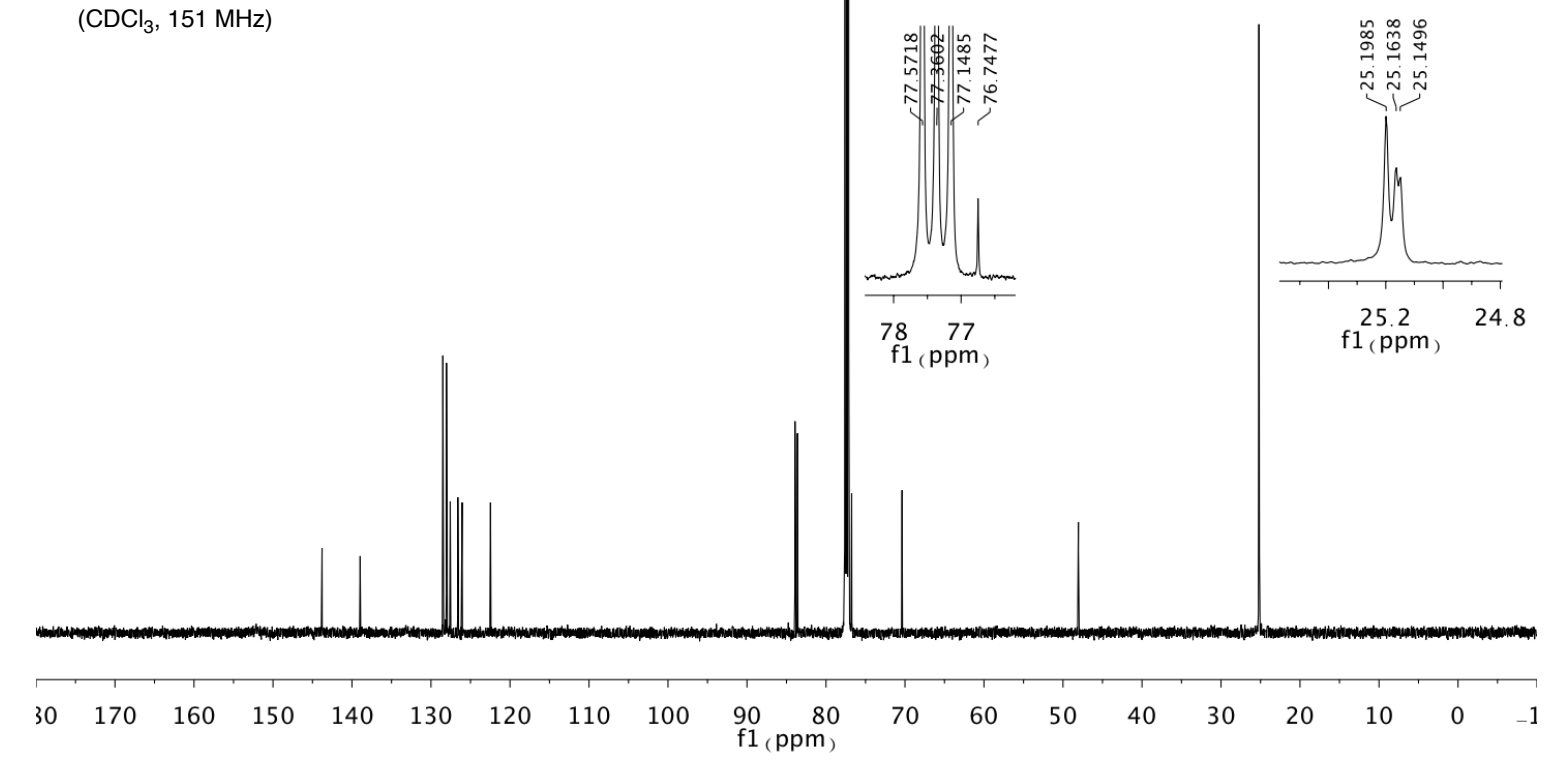



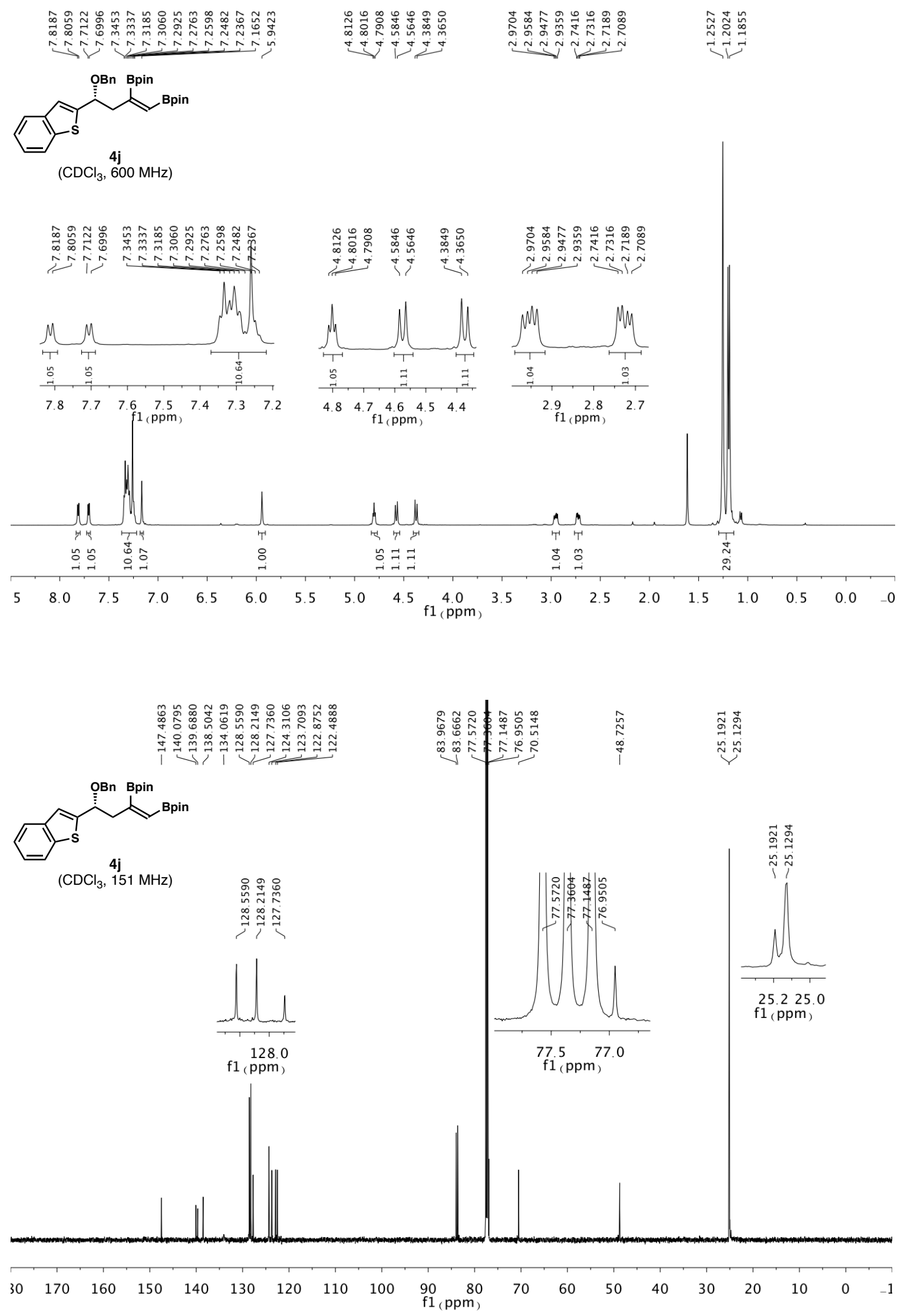

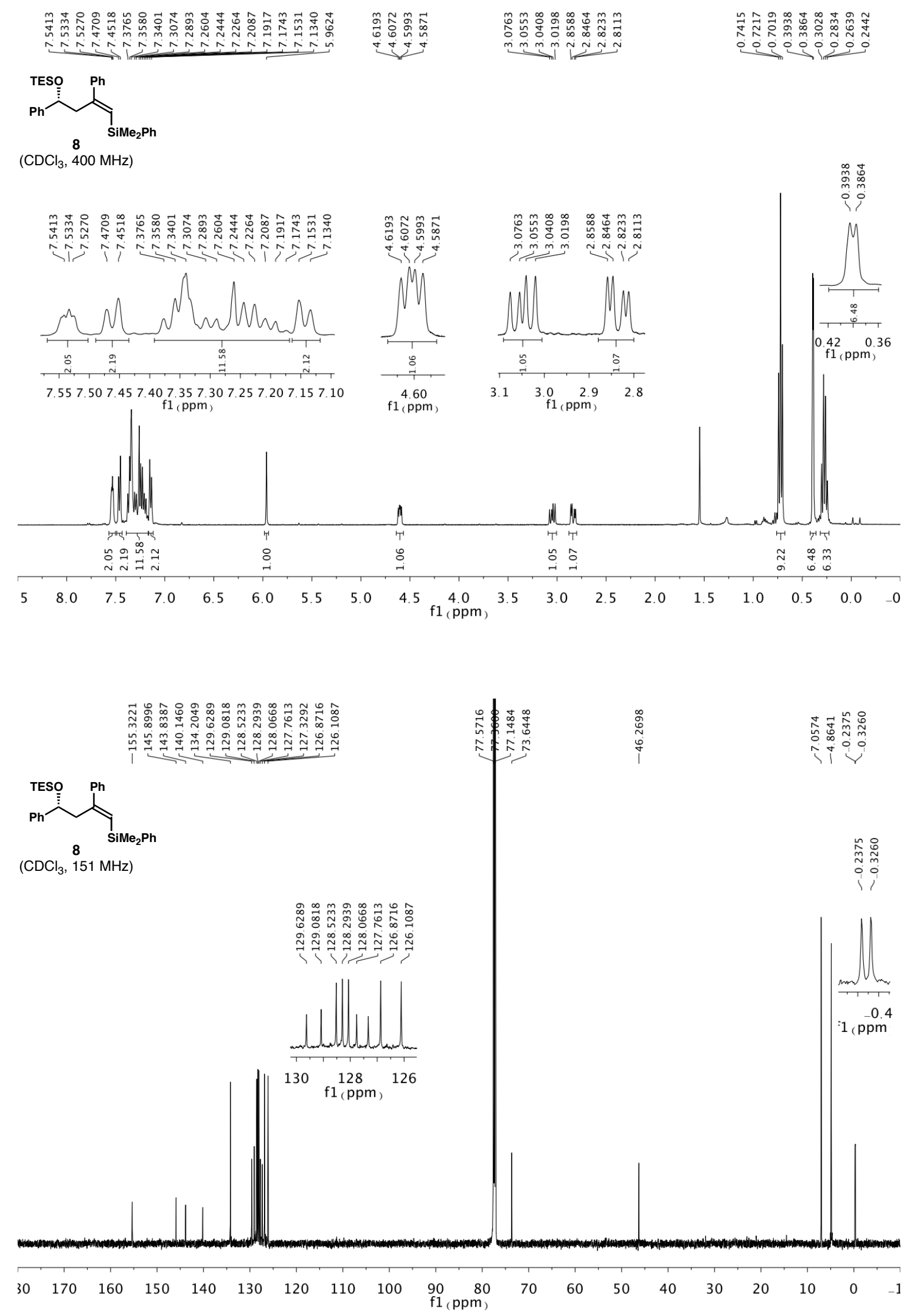

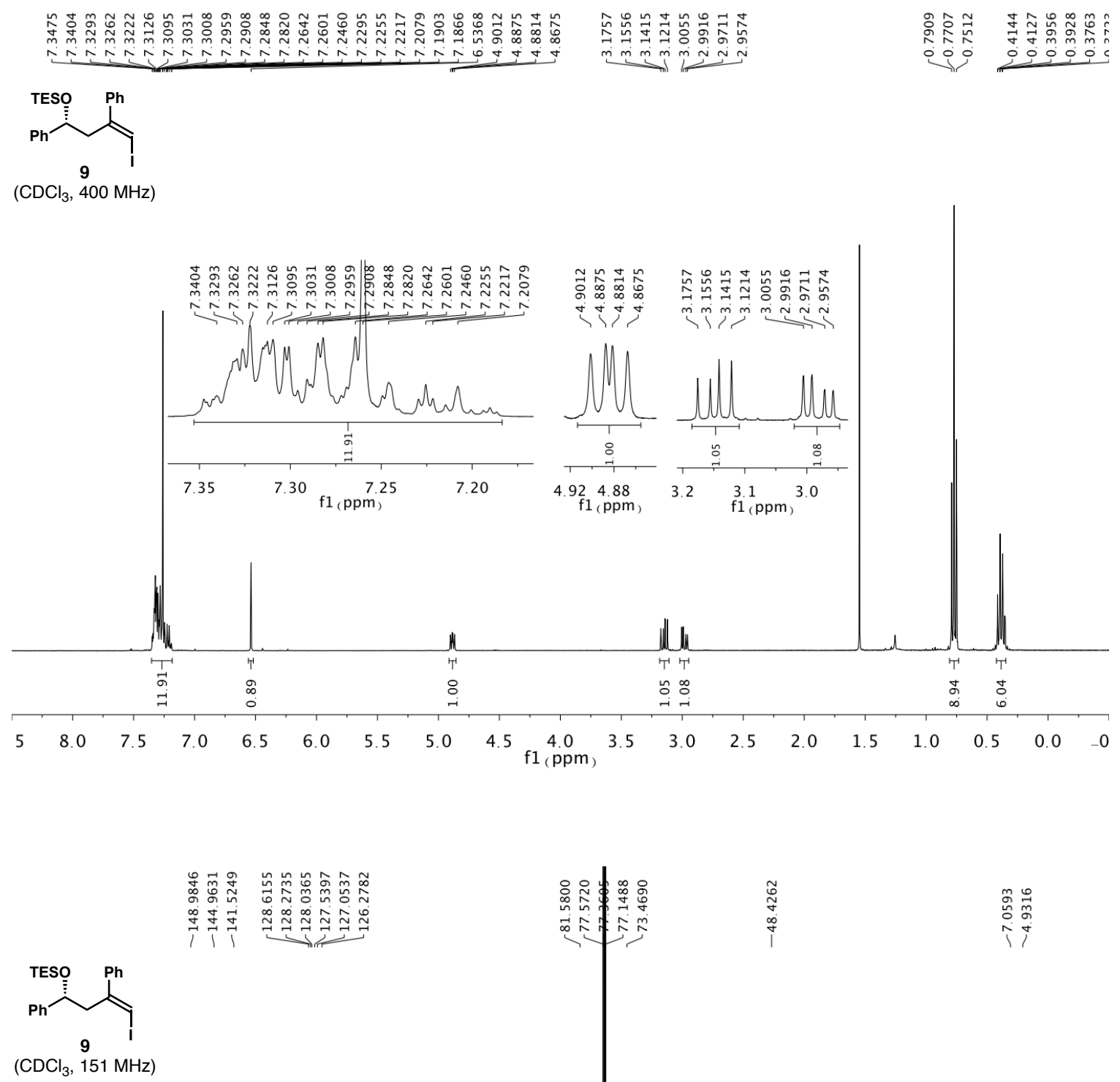

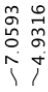

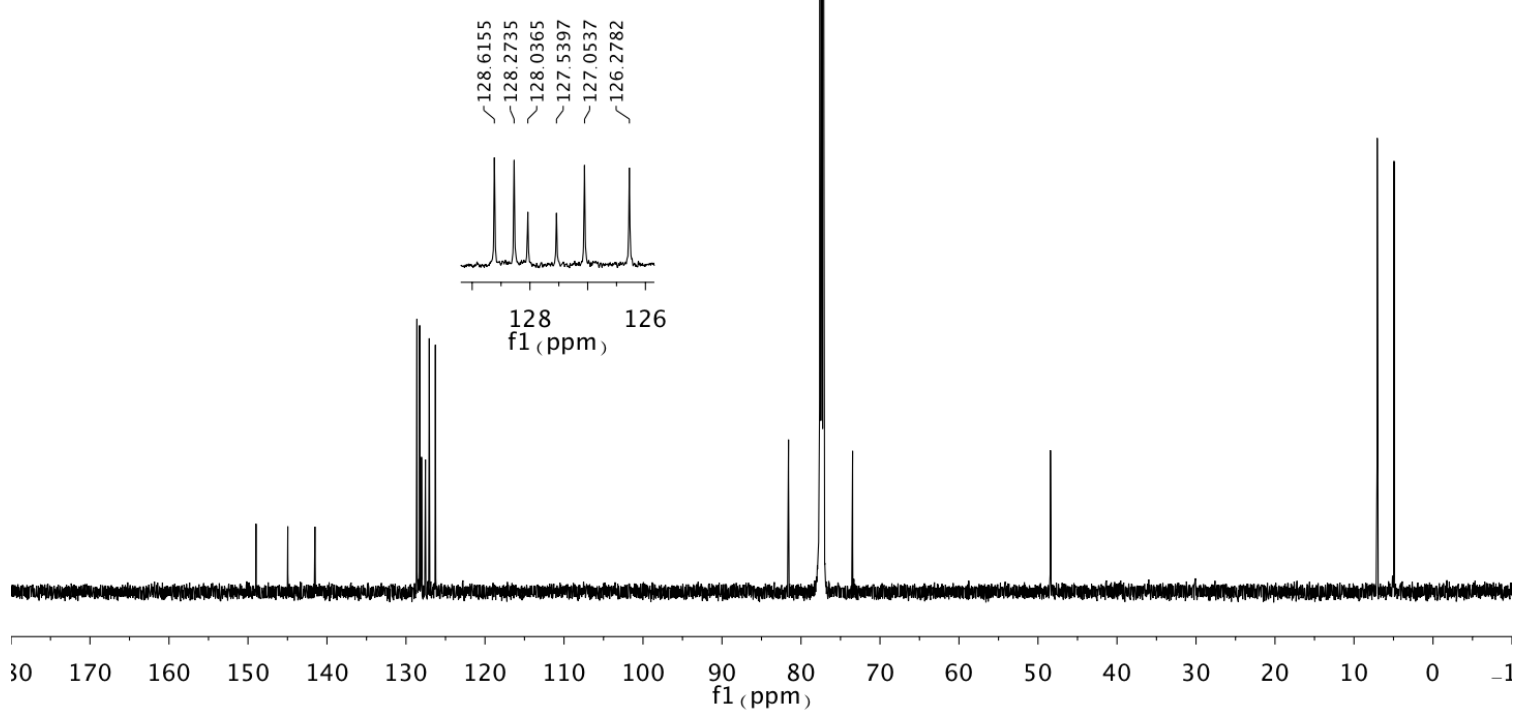



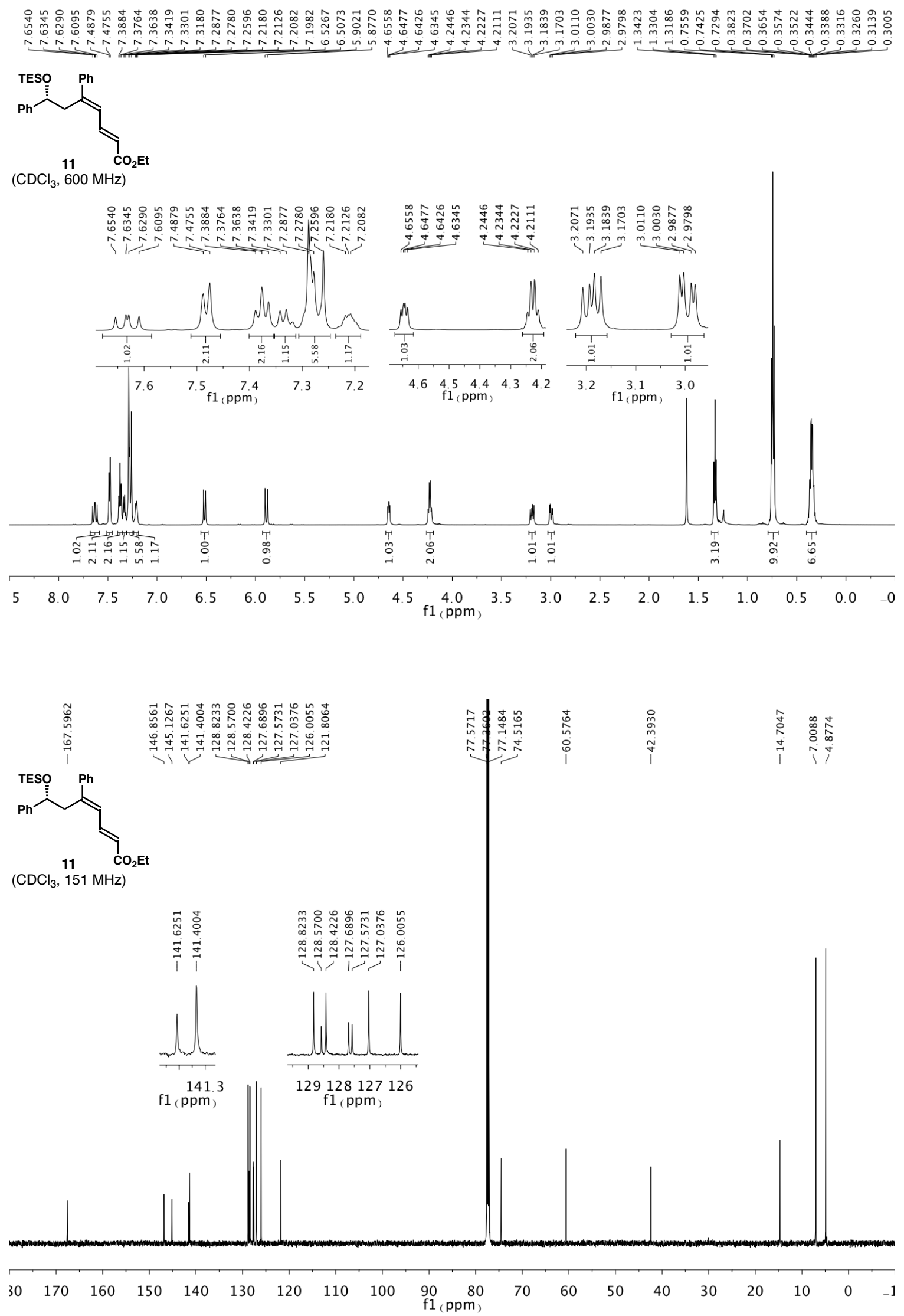

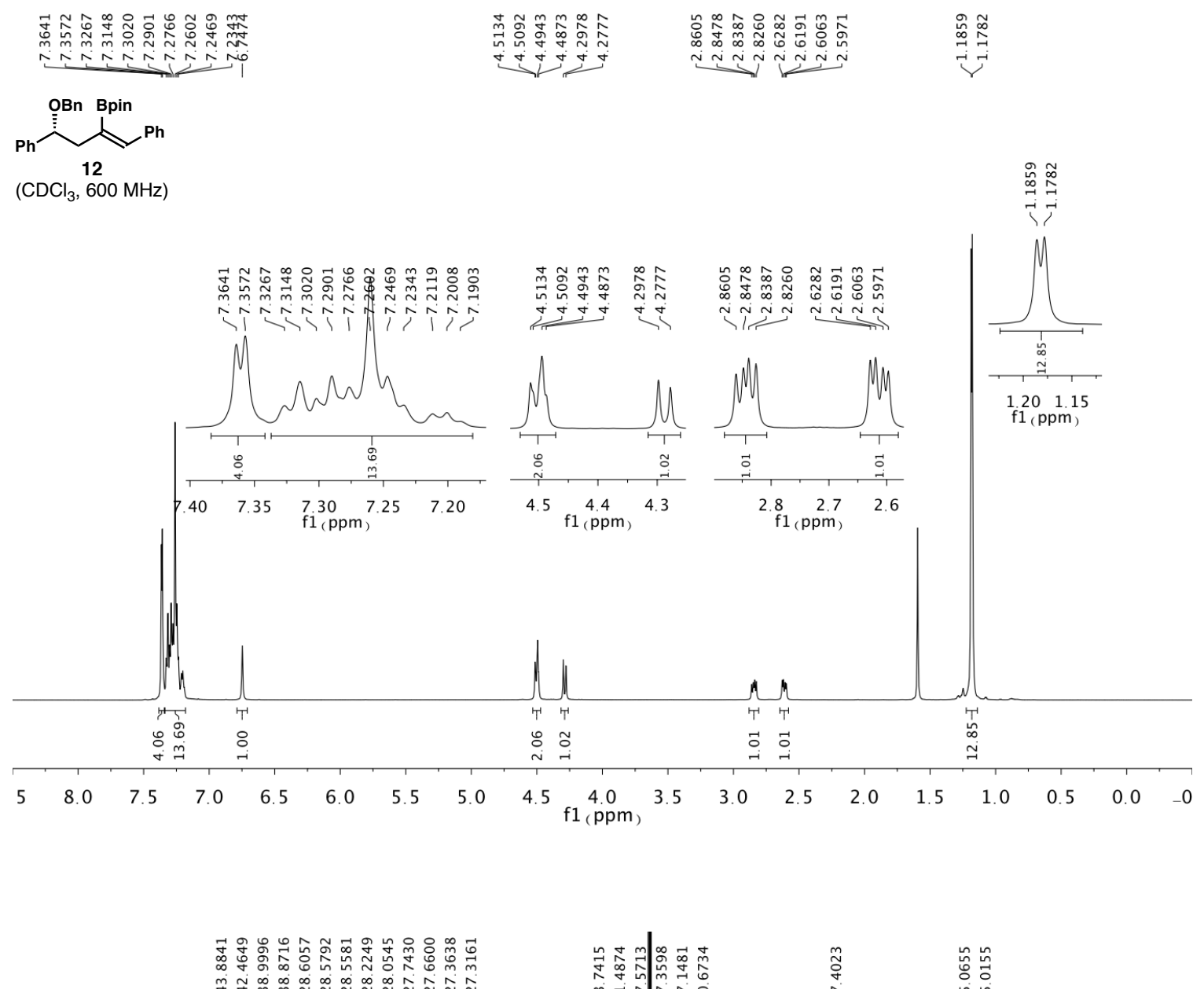

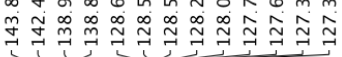
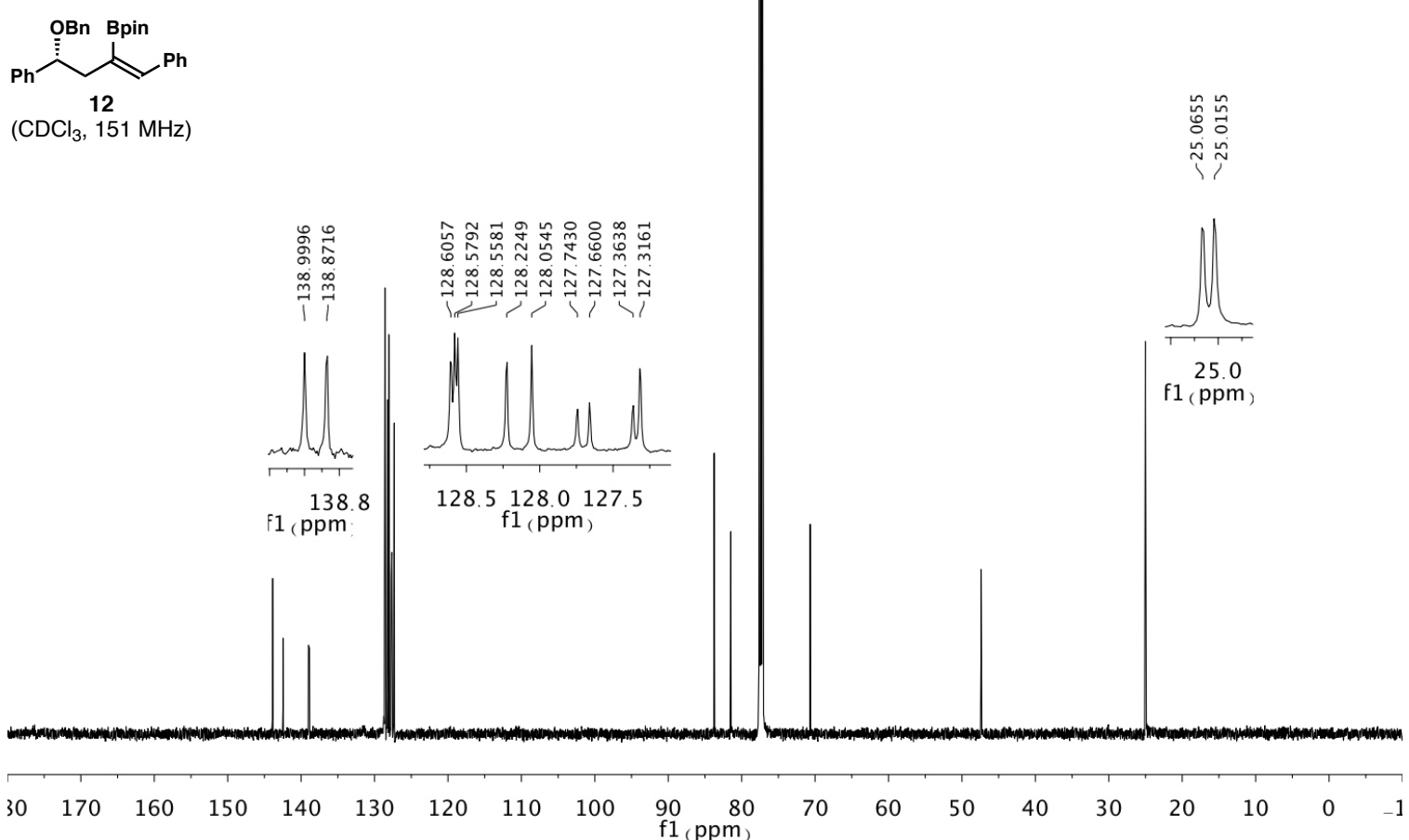

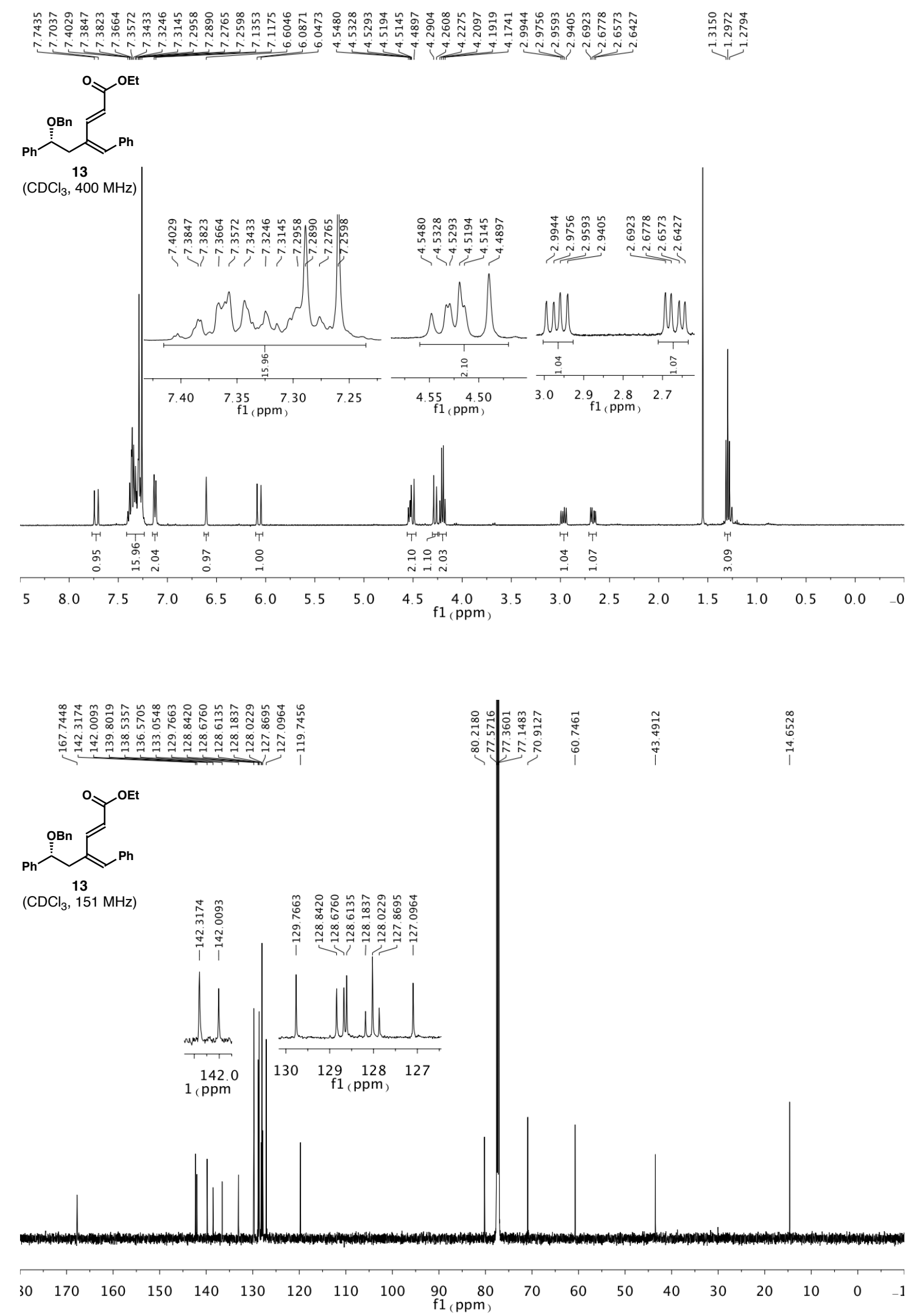


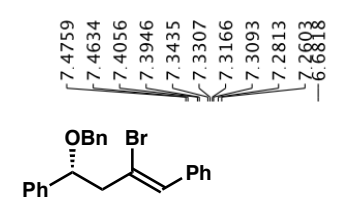

14

$\left(\mathrm{CDCl}_{3}, 600 \mathrm{MHz}\right)$
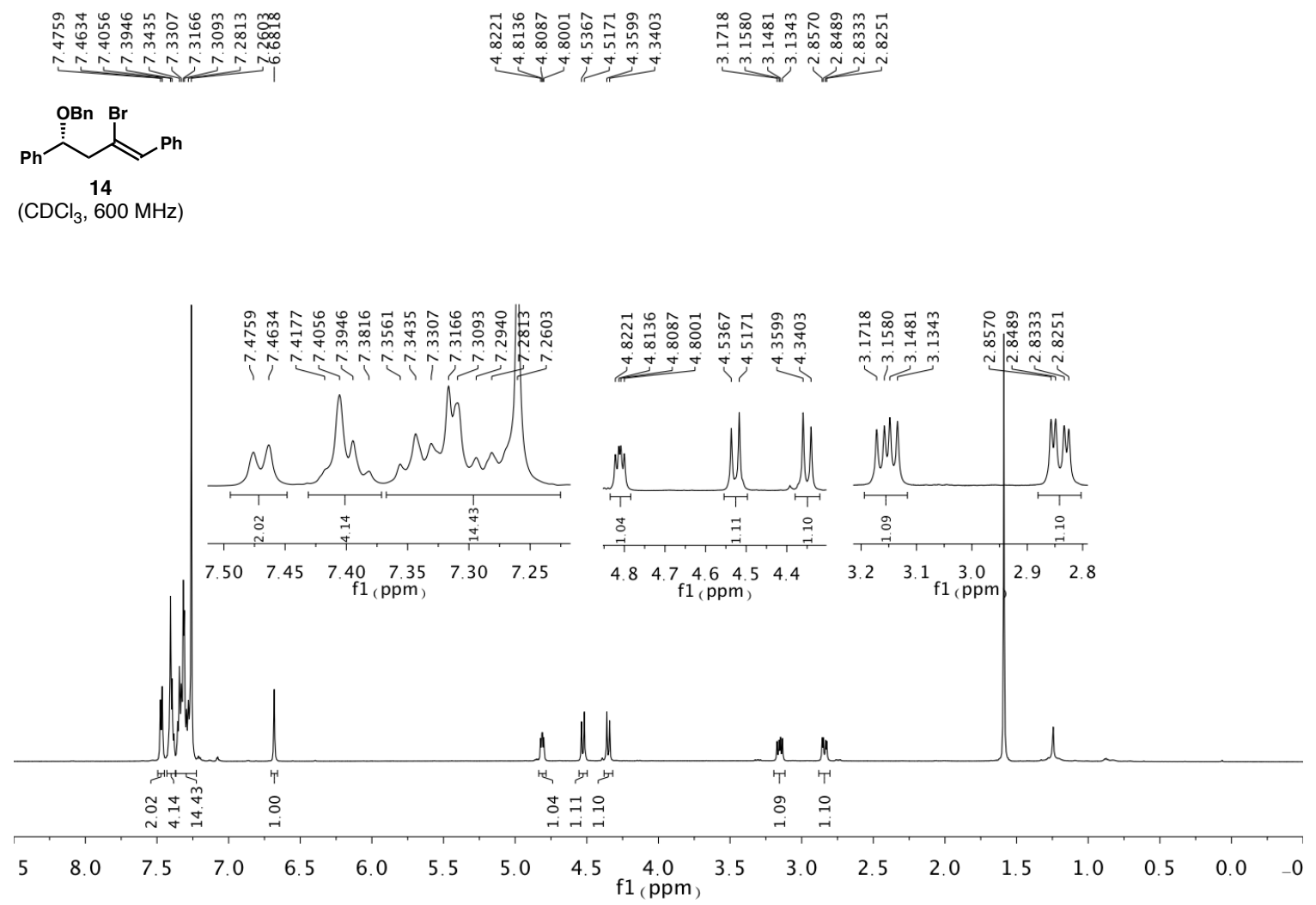

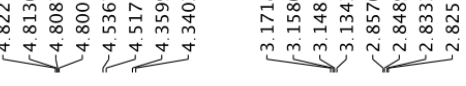

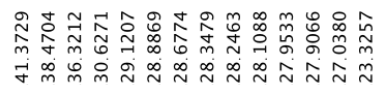

$\exists$
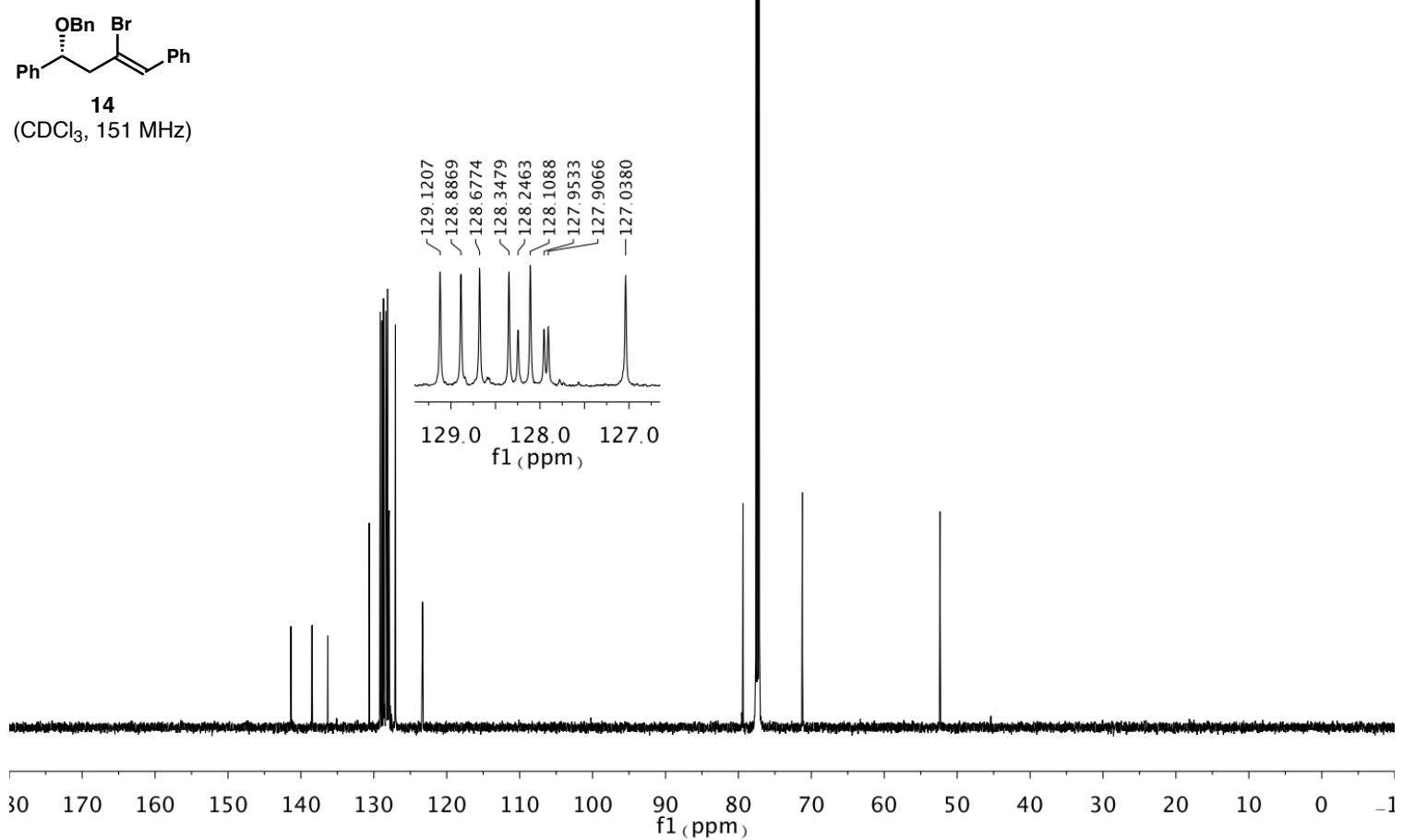


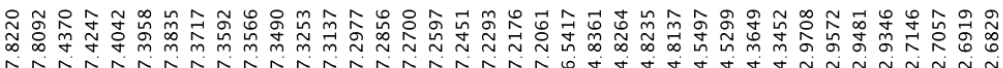
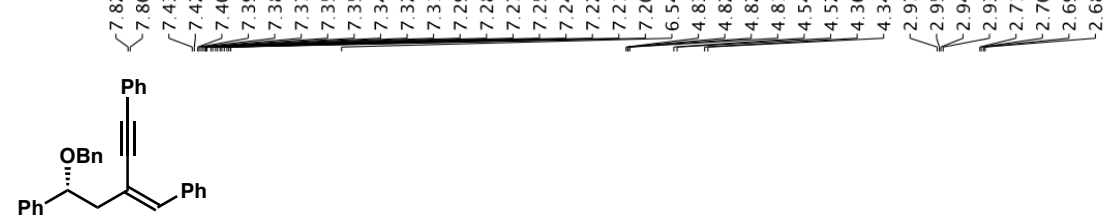

15

$\left(\mathrm{CDCl}_{3}, 600 \mathrm{MHz}\right)$
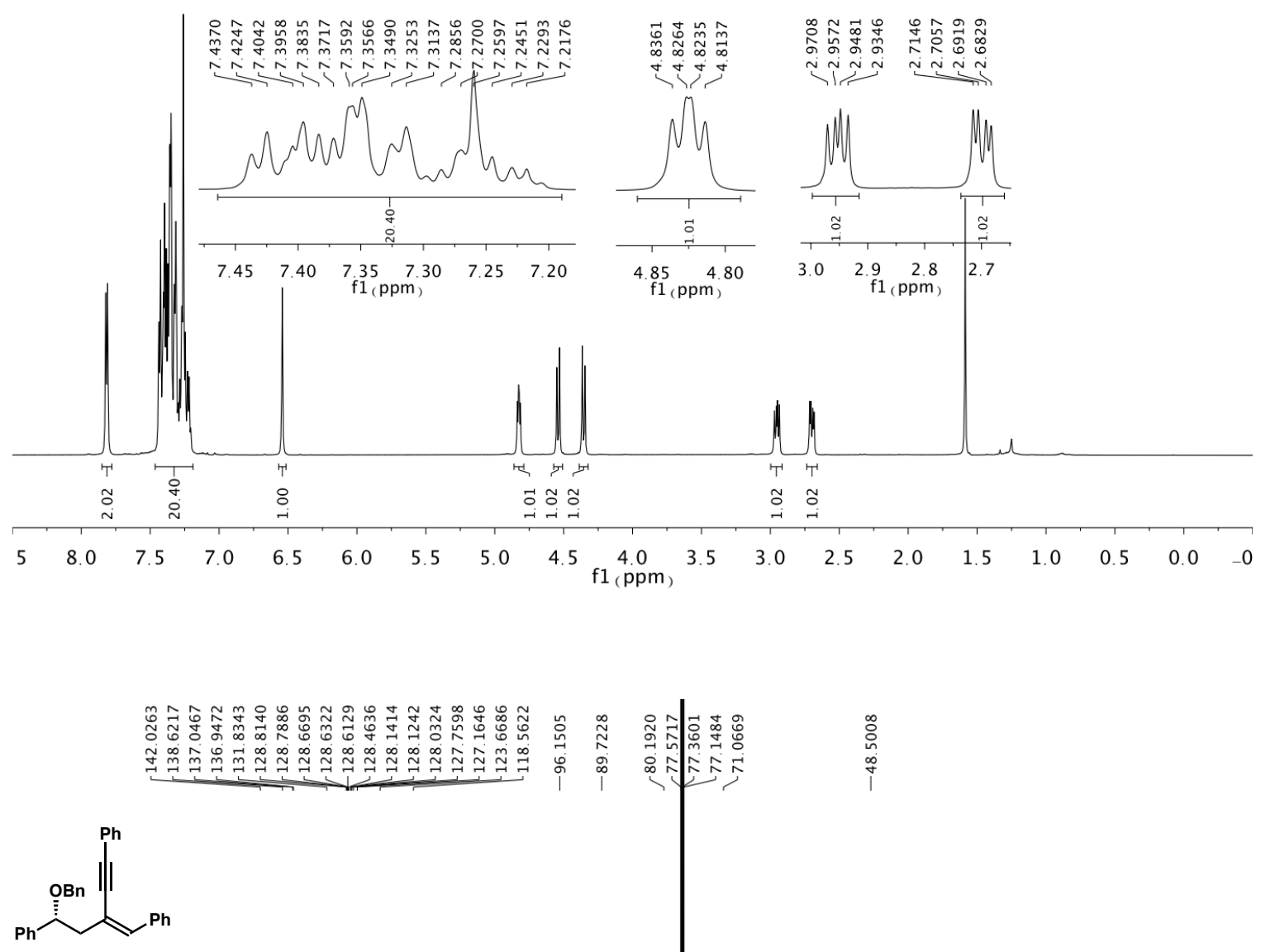

$\left(\mathrm{CDCl}_{3}, 151 \mathrm{MHz}\right)$

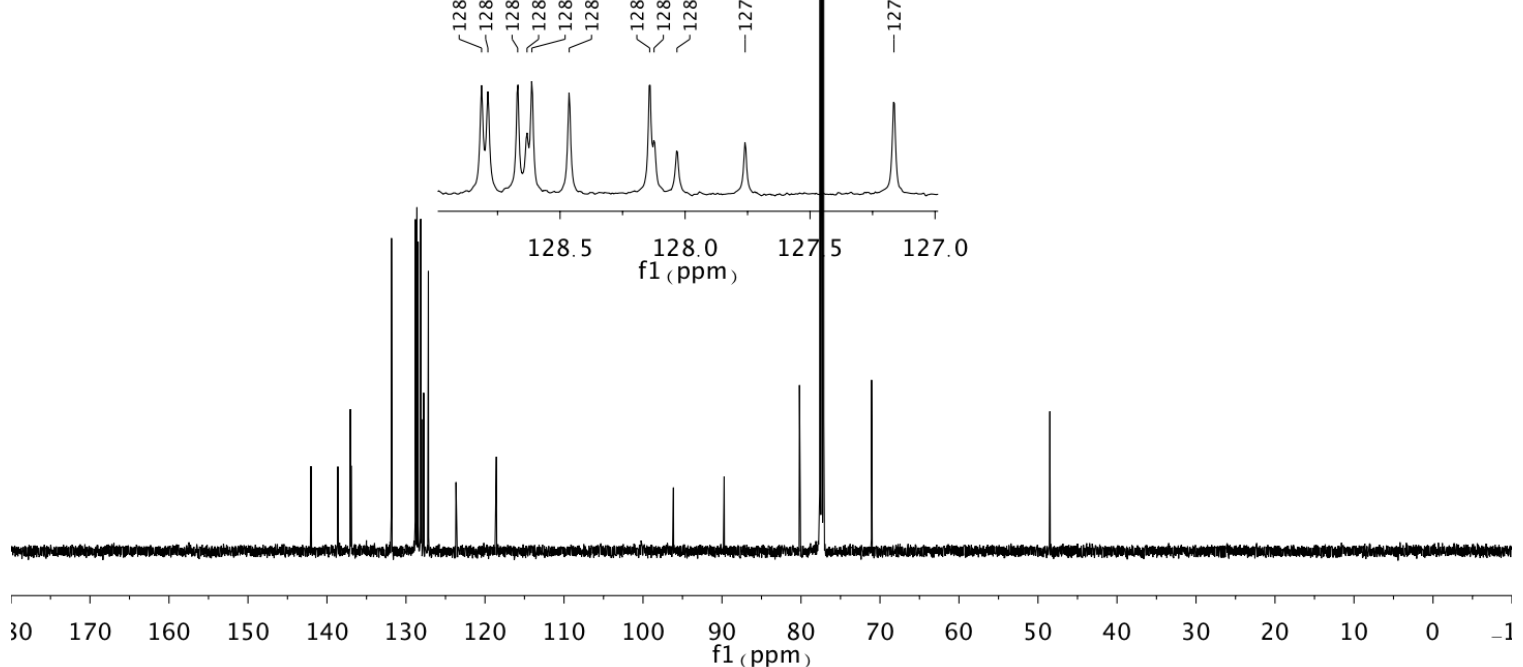

